\title{
Constructions of representations of rank two semisimple Lie algebras with distributive lattices
}

\author{
L. Wyatt Alverson II \\ leslie.alverson@murraystate.edu \\ Scott J. Lewis \\ scott.lewis@murraystate.edu
}

\author{
Robert G. Donnelly \\ rob.donnelly@murraystate.edu \\ Robert Pervine \\ bob.pervine@murraystate.edu
}

\author{
Department of Mathematics and Statistics \\ Murray State University, Murray, KY 42071 USA
}

Submitted: Aug 20, 2006; Accepted: Nov 14, 2006; Published: Nov 23, 2006

Mathematics Subject Classification: 05E15

\begin{abstract}
We associate one or two posets (which we call "semistandard posets") to any given irreducible representation of a rank two semisimple Lie algebra over $\mathbb{C}$. Elsewhere we have shown how the distributive lattices of order ideals taken from semistandard posets (we call these "semistandard lattices") can be used to obtain certain information about these irreducible representations. Here we show that some of these semistandard lattices can be used to present explicit actions of Lie algebra generators on weight bases (Theorem 5.1), which implies these particular semistandard lattices are supporting graphs. Our descriptions of these actions are explicit in the sense that relative to the bases obtained, the entries for the representing matrices of certain Lie algebra generators are rational coefficients we assign in pairs to the lattice edges. In Theorem 4.4 we show that if such coefficients can be assigned to the edges, then the assignment is unique up to products; we conclude that the associated weight bases enjoy certain uniqueness and extremal properties (the "solitary" and "edge-minimal" properties respectively). Our proof of this result is uniform and combinatorial in that it depends only on certain properties possessed by all semistandard posets. For certain families of semistandard lattices some of these results were obtained in previous papers; in Proposition 5.6 we explicitly construct new weight bases for a certain family of rank two symplectic representations. These results are used to help obtain in Theorem 5.1 the classification of those semistandard lattices which are supporting graphs.
\end{abstract}

Keywords: distributive lattice, rank two semisimple Lie algebra, irreducible representation, weight basis, supporting graph, solitary basis, edge-minimal basis

1. Introduction

\section{Contents}

2. Definitions and preliminary results

3. Grid posets; two-color grid posets; semistandard posets and lattices

4. Semistandard lattices as supporting graphs

5. Classification of semistandard lattice supporting graphs

6. An additional example 


\section{Introduction}

The main questions this paper seeks to address are (1) whether the four families of "semistandard" distributive lattices introduced in [ADLMPW] can be used to concretely realize the irreducible representations of the rank two semisimple Lie algebras $A_{1} \times A_{1}$, $A_{2}, C_{2}$, and $G_{2}$, and (2) what properties such concrete realizations derive from the combinatorics of the lattices. Our four families of semistandard lattices are indexed by the algebras $A_{1} \times A_{1}, A_{2}, C_{2}$, and $G_{2}$ and are each parameterized by pairs of nonnegative integers $(a, b)$; for a given algebra and a given pair of nonzero integers there are one or two semistandard lattices. Their posets of join irreducibles also play an important role in our development and are called semistandard posets. With one exception (an observation recorded here as Proposition 4.7), the results of this paper are independent of the main character result (Theorem 5.3) of [ADLMPW]. Indeed one of our goals at the outset was to recover this result as a consequence of our work in answering question (1); our partial success is recorded in Corollary 5.3 below.

For question (1), we would like to construct an irreducible representation of a given rank two semisimple Lie algebra by using elements of an appropriate semistandard lattice as basis vectors for a representing space. We require that the basis indexed by lattice elements be a weight basis, so in particular each basis vector should be an eigenvector under the actions of certain Lie algebra elements (elements of a specified Cartan subalgebra). We would also like lattice edges to tell us the locations of nonzero entries for representing matrices for certain other Lie algebra generators (the Chevalley generators $x_{i}$ and $y_{i}$ ). We will view such matrix entries as coefficients attached to the lattice edges, with two coefficients per edge. The coefficients must satisfy certain relations that are combinatorial versions of the Serre relations. (The combinatorial constructions here follow the approach described in [Don1] of obtaining explicit descriptions of actions of a generating set for the Lie algebra; in [Wil1] and [Wil2], actions of a basis for the Lie algebra are sought.) Although each semistandard lattice can generate the appropriate Weyl character in a nice fashion, it turns out that only some of these lattices can carry the desired representation. In Section 5 we also obtain or say how to obtain explicit formulas for the coefficients/matrix entries, and when possible we connect these constructions with others in the literature. When such coefficients/matrix entries can be found, the lattice can be called (following [Don1]) a "supporting graph" for a representation of the appropriate rank two semisimple Lie algebra. In Theorem 5.1 we completely classify which semistandard lattices are supporting graphs.

However, before resolving the question of the existence of such realizations, we will address question (2) first. We are interested in two properties of weight bases and supporting graphs: the solitary and edge-minimal properties. These notions were introduced in [Don1] and studied further in [DLP1], [DLP2], and [Don2]. The solitary property is a uniqueness property: a weight basis is solitary if all weight bases which share its supporting graph are the same (up to a certain notion of scalar equivalence). The edge-minimal property is an extremal property: a weight basis is edge-minimal if its supporting graph does not contain as a proper edge-colored subgraph the supporting graph for any other 
weight basis for the same representation. We apply a method obtained in [DLP2] which says that when a supporting graph meets certain combinatorial requirements, then the product of the two coefficients on any given edge is uniquely determined and that the weight basis for the representation is solitary and edge-minimal. This leads to our answer of question (2) in Theorem 4.4: if a semistandard lattice is a supporting graph for a representation of a rank two semisimple Lie algebra, then it is solitary and edge-minimal. This result is uniform across the type of the Lie algebra; in particular, it only depends on certain combinatorial properties shared by all semistandard lattices, and not on the classification of Theorem 5.1.

Semistandard posets and lattices have other combinatorial virtues and connections to the representation theory of rank two semisimple Lie algebras. It was shown in [ADLMPW] that the Weyl characters for the irreducible representations of the rank two semisimple Lie algebras can be expressed as certain weight generating functions on our semistandard lattices. From this one can derive nice quotient-of-products expressions for their rank generating functions, obtain closed formulas for the number of lattice elements, and deduce that the sequence of coefficients for the monomial terms of the rank generating function in each case is symmetric and unimodal. Certain combinatorial properties shared by all semistandard posets were used to effect a uniform presentation of results in [ADLMPW]; here certain other combinatorial properties derived in Section 3 are used to obtain the uniqueness result Theorem 4.4. One of us (Donnelly) has shown that semistandard posets are uniquely characterized by a short list of abstract combinatorial properties [Don3]; these are precisely the properties that are used to obtain the type-independent results of both papers.

In Section 2, we develop language, fix notation, recapitulate results from previous papers, and derive some new results that will be useful not only here but also in future papers that seek to extend results of this paper. Throughout Section 2 are examples that concretely illustrate the ideas we use. The reader could browse this section at the outset and consult as needed along the way. Following [ADLMPW], in Section 3 we revisit the notion of a two-color grid poset and derive two general lemmas (Lemmas 3.1 and 3.2) that will be applied to semistandard posets and semistandard lattices to obtain the main result of Section 4 (Theorem 4.4). In Section 5 we say precisely which semistandard lattices are supporting graphs. In Propositions 5.5 and 5.6 we give constructions over $\mathbb{Z}$ and $\mathbb{Q}$ respectively of bases for two infinite families of irreducible representations of the rank two symplectic Lie algebra; bases for one of these constructions appear to be new (Proposition 5.6). Propositions 5.6, 5.7, and 5.8 were discovered with the aid of computer programs written by Alverson as part of a Master's thesis [Alv]. Section 6 contains a reference example. In addition to $G_{2}$ and $A_{2} \cong \mathfrak{s l}(3, \mathbb{C})$, the remaining rank two simple Lie algebra will be referred to as $C_{2}$ (corresponding to the symplectic Lie algebra $\mathfrak{s p}(4, \mathbb{C})$ ) rather than $B_{2}$ (corresponding to $\mathfrak{s o}(5, \mathbb{C})$, which is isomorphic to $\mathfrak{s p}(4, \mathbb{C})$ ). We do so in part because we believe the combinatorics of the presentation here for $C_{2}$ extends more naturally to the $C_{n}$ series, and in part to avoid confusion with the $B_{2}$ constructions of [DLP1] (for example, the "one-rowed" representations of $B_{2}$ studied there are not the same as the "one-rowed" representations of $C_{2}$ considered in here Proposition 5.5). 
Acknowledgment The authors thank Bob Proctor for his helpful feedback and suggestions.

\section{Definitions and preliminary results}

Some of the definitions and notational conventions of this section borrow from [Don1], [DLP1], [DLP2], and [ADLMPW]; we include them here for the reader's convenience. Our main combinatorics reference is [Sta]; for the representation theory of semisimple Lie algebras, see $[\mathrm{Hum}]$. We use " $R$ " (and when necessary, " $Q$ ") as a generic name for most of the combinatorial objects we define in this section ("edge-colored directed graph," "vertex-colored directed graph," "ranked poset," "edge-labelled poset," "supporting graph," "representation diagram"). The letter " $P$ " is reserved for posets (and "vertex-colored" posets) that will be viewed as posets of join irreducibles for distributive lattices; we reserve use of the letter " $L$ " for reference to distributive lattices and "edge-colored" distributive lattices.

Let $I$ be any set. An edge-colored directed graph with edges colored by the set $I$ is a directed graph $R$ with vertex set $\mathcal{V}(R)$ and directed-edge set $\mathcal{E}(R)$ together with a function edgecolor $_{R}: \mathcal{E}(R) \longrightarrow I$ assigning to each edge of $R$ an element ("color") from the set

$I$. If an edge $\mathbf{s} \rightarrow \mathbf{t}$ in $R$ is assigned color $i \in I$, we write $\mathbf{s} \stackrel{i}{\rightarrow} \mathbf{t}$. For $i \in I$, we let $\mathcal{E}_{i}(R)$ denote the set of edges in $R$ of color $i$, so $\mathcal{E}_{i}(R)=\operatorname{edgecolor}_{R}^{-1}(i)$. If $J$ is a subset of $I$, remove all edges from $R$ whose colors are not in $J$; connected components of the resulting edge-colored directed graph are called $J$-components of $R$. For any $\mathbf{t}$ in $R$ and any $J \subset I$, we let $\operatorname{comp}_{J}(\mathbf{t})$ denote the $J$-component of $R$ containing $\mathbf{t}$. The $d u a l R^{*}$ is the edge-colored directed graph whose vertex set $\mathcal{V}\left(R^{*}\right)$ is the set of symbols $\left\{\mathbf{t}^{*}\right\}_{\mathbf{t} \in R}$ together with colored edges $\mathcal{E}_{i}\left(R^{*}\right):=\left\{\mathbf{t}^{*} \stackrel{i}{\rightarrow} \mathbf{s}^{*} \mid \mathbf{s} \stackrel{i}{\rightarrow} \mathbf{t} \in \mathcal{E}_{i}(R)\right\}$ for each $i \in I$. Let $Q$ be another edge-colored directed graph with edge colors from $I$. If $R$ and $Q$ have disjoint vertex sets, then the disjoint sum $R \oplus Q$ is the edge-colored directed graph whose vertex set is the disjoint union $\mathcal{V}(R) \cup \mathcal{V}(Q)$ and whose colored edges are $\mathcal{E}_{i}(R) \cup \mathcal{E}_{i}(Q)$ for each $i \in I$. If $\mathcal{V}(Q) \subseteq \mathcal{V}(R)$ and $\mathcal{E}_{i}(Q) \subseteq \mathcal{E}_{i}(R)$ for each $i \in I$, then $Q$ is an edge-colored subgraph of $R$. Let $R \times Q$ denote the edge-colored directed graph whose vertex set is the Cartesian product $\{(\mathbf{s}, \mathbf{t}) \mid \mathbf{s} \in R, \mathbf{t} \in Q\}$ and with colored edges $\left(\mathbf{s}_{1}, \mathbf{t}_{1}\right) \stackrel{i}{\rightarrow}\left(\mathbf{s}_{2}, \mathbf{t}_{2}\right)$ if and only if $\mathbf{s}_{1}=\mathbf{s}_{2}$ in $R$ with $\mathbf{t}_{1} \stackrel{i}{\rightarrow} \mathbf{t}_{2}$ in $Q$ or $\mathbf{s}_{1} \stackrel{i}{\rightarrow} \mathbf{s}_{2}$ in $R$ with $\mathbf{t}_{1}=\mathbf{t}_{2}$ in $Q$. Two edgecolored directed graphs are isomorphic if there is a bijection between their vertex sets that preserves edges and edge colors. If $R$ is an edge-colored directed graph with edges colored by the set $I$, and if $\sigma: I \longrightarrow I^{\prime}$ is a mapping of sets, then we let $R^{\sigma}$ be the edge-colored directed graph with edge color function edgecolor $R^{\sigma}:=\sigma \circ$ edgecolor $_{R}$. We call $R^{\sigma}$ a recoloring of $R$. Observe that $\left(R^{*}\right)^{\sigma} \cong\left(R^{\sigma}\right)^{*}$. We similarly define a vertex-colored directed graph with a function vertexcolor $R: \mathcal{V}(R) \longrightarrow I$ that assigns colors to the vertices of $R$. In this context, we speak of the dual vertex-colored directed graph $R^{*}$, the disjoint sum of two vertex-colored directed graphs with disjoint vertex sets, isomorphism of vertex-colored directed graphs, recoloring, etc. See Figures 2.1, 2.2, and 2.3 for examples.

In this paper, we identify a poset with its Hasse diagram ([Sta] p. 98), and all posets will be finite. For elements $\mathbf{s}$ and $\mathbf{t}$ of a poset $R$, there is a directed edge $\mathbf{s} \rightarrow \mathbf{t}$ in the 
Figure 2.1: A vertex-colored poset $P$ and an edge-colored lattice $L$.

(The set of vertex colors for $P$ and the set of edge colors for $L$ are $\{\alpha, \beta\}$.

Elements of $P$ are denoted $v_{i}$ and elements of $L$ are denoted $\mathbf{t}_{i}$.)
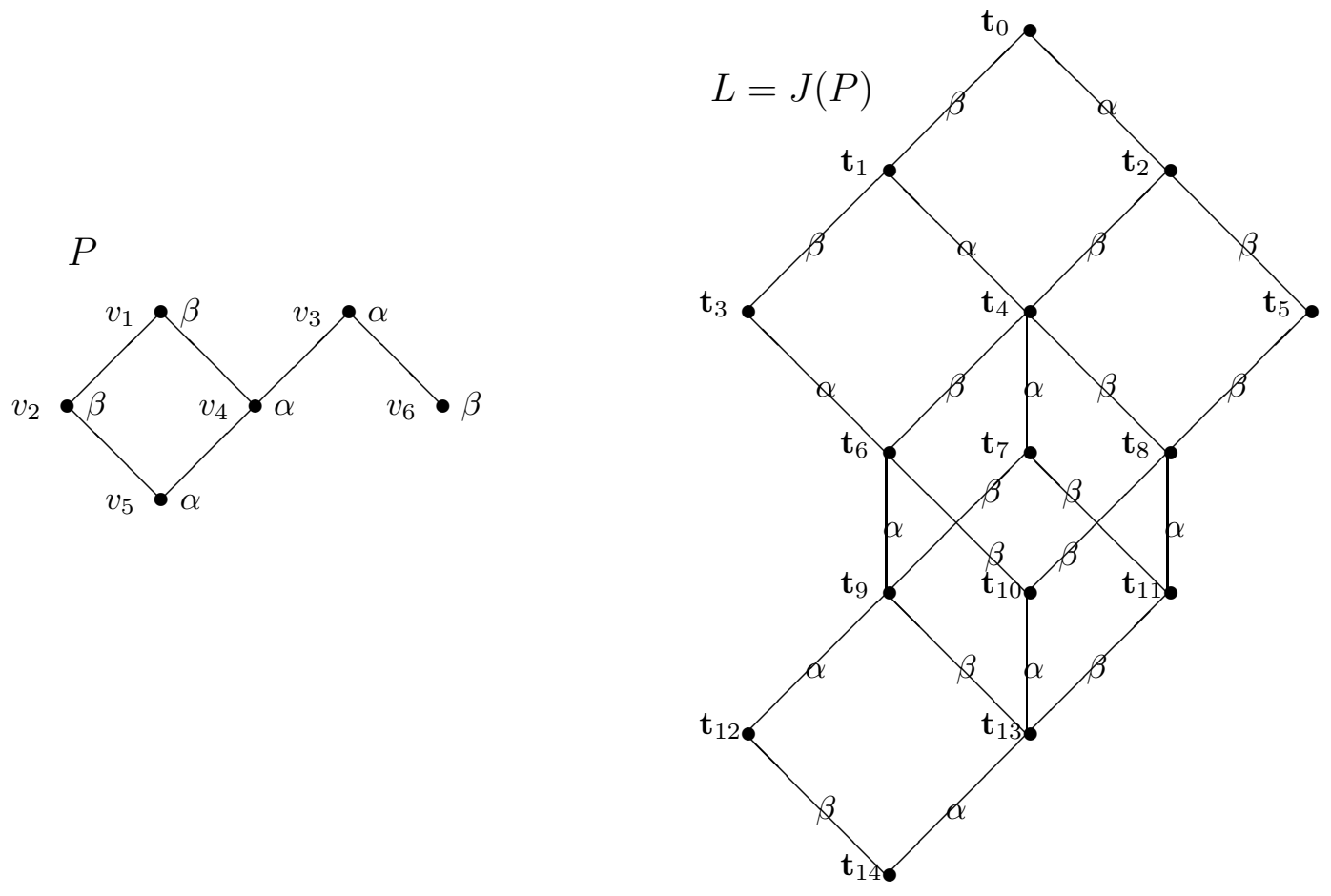

Hasse diagram if and only if $\mathbf{t}$ covers $\mathbf{s}$, i.e. $\mathbf{s}<\mathbf{t}$ and there is no $\mathbf{x}$ in $R$ such that $\mathbf{s}<\mathbf{x}<\mathbf{t}$. Thus, terminology (connected, edge-colored, dual, vertex-colored, etc) that applies to directed graphs will also apply to posets. When we depict the Hasse diagram for a poset, its edges are directed "up." In an edge-colored poset $R$, we say the vertex $\mathbf{s}$ and the edge $\mathbf{s} \stackrel{i}{\rightarrow} \mathbf{t}$ are below $\mathbf{t}$, and the vertex $\mathbf{t}$ and the edge $\mathbf{s} \stackrel{i}{\rightarrow} \mathbf{t}$ are above $\mathbf{s}$. The vertex $\mathbf{s}$ is a descendant of $\mathbf{t}$, and $\mathbf{t}$ is an ancestor of $\mathbf{s}$. The edge-colored and vertexcolored directed graphs studied in this paper will turn out to be posets. For a directed graph $R$, a rank function is a surjective function $\rho: R \longrightarrow\{0, \ldots, l\}$ (where $l \geq 0$ ) with the property that if $\mathbf{s} \rightarrow \mathbf{t}$ in $R$, then $\rho(\mathbf{s})+1=\rho(\mathbf{t})$; if such a rank function exists then $R$ is the Hasse diagram for a poset - a ranked poset. We call $l$ the length of $R$ with respect to $\rho$, and the set $\rho^{-1}(i)$ is the $i$ th rank of $R$. In an edge-colored ranked poset $R$, $\operatorname{comp}_{i}(\mathbf{t})$ will be a ranked poset for each $\mathbf{t} \in R$ and $i \in I$. We let $l_{i}(\mathbf{t})$ denote the length of $\operatorname{comp}_{i}(\mathbf{t})$, and we let $\rho_{i}(\mathbf{t})$ denote the rank of $\mathbf{t}$ within this component. We define the depth of $\mathbf{t}$ in its $i$-component to be $\delta_{i}(\mathbf{t}):=l_{i}(\mathbf{t})-\rho_{i}(\mathbf{t})$.

For distributive lattices we follow the notation and language of [Sta]. In particular, the distributive lattice of order ideals taken from a poset $P$ (partially ordered by subset containment) will be denoted $J(P)$, and we use $\mathbf{s} \vee \mathbf{t}$ and $\mathbf{s} \wedge \mathbf{t}$ to denote the least upper bound ("join") and greatest lower bound ("meet") respectively for two elements $\mathbf{s}$ and $\mathbf{t}$ of the distributive lattice $J(P)$. If we regard the Hasse diagram for $L$ to be an undirected 
Figure 2.2: $L^{*}$ and $\left(L^{*}\right)^{\sigma}$ for the lattice $L$ from Figure 2.1.

(Here $\sigma(\alpha)=1$ and $\sigma(\beta)=2$.)
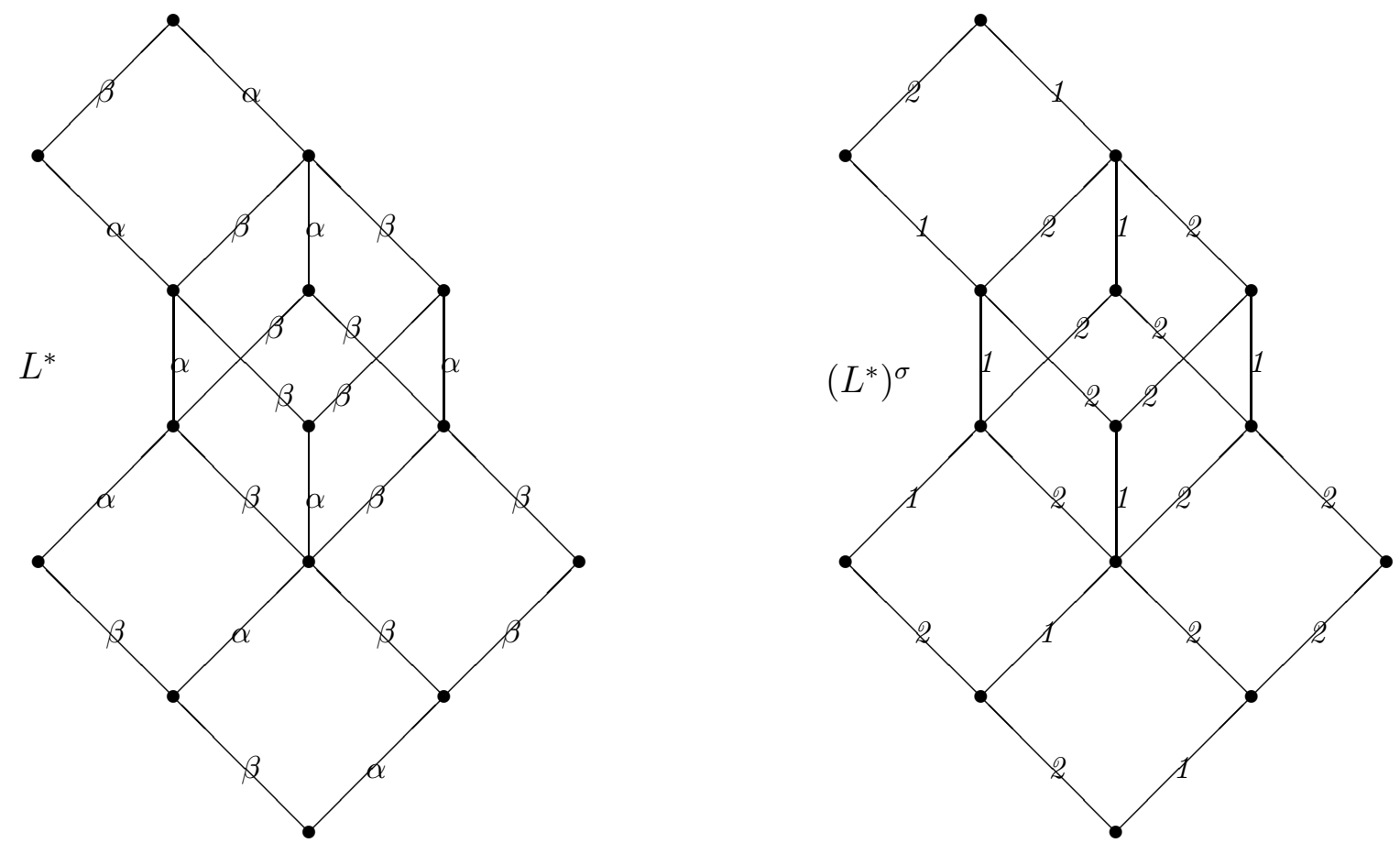

graph, the we define the distance $\operatorname{dist}(\mathbf{s}, \mathbf{t})$ between $\mathbf{s}$ and $\mathbf{t}$ in $L$ to be the minimum length achieved when all paths from $\mathbf{s}$ to $\mathbf{t}$ in $L$ are considered; it can be seen that $\operatorname{dist}(\mathbf{s}, \mathbf{t})=2 \rho(\mathbf{s} \vee \mathbf{t})-\rho(\mathbf{s})-\rho(\mathbf{t})=\rho(\mathbf{s})+\rho(\mathbf{t})-2 \rho(\mathbf{s} \wedge \mathbf{t})$. A coloring of the vertices of the poset $P$ gives a natural coloring of the edges of the distributive lattice $L=J(P)$ in the following way: Given a function vertexcolor $_{P}: \mathcal{V}(P) \longrightarrow I$ which assigns to each vertex of $P$ a color from the target set $I$, then we give a covering relation $\mathbf{s} \rightarrow \mathbf{t}$ in $L$ the color $i$ and write $\mathbf{s} \stackrel{i}{\rightarrow} \mathbf{t}$ if $\mathbf{t} \backslash \mathbf{s}=\{u\}$ and vertexcolor $_{P}(u)=i$. So we can regard $L$ to be an edge-colored distributive lattice with edges colored by the set $I$; for brevity, we write $L=J_{\text {color }}(P)$. See Figure 2.4 for an example. Note that $J_{\text {color }}\left(P^{*}\right) \cong\left(J_{\text {color }}(P)\right)^{*}$, $J_{\text {color }}\left(P^{\sigma}\right) \cong\left(J_{\text {color }}(P)\right)^{\sigma}$ (recoloring), and $J_{\text {color }}(P \oplus Q) \cong J_{\text {color }}(P) \times J_{\text {color }}(Q)$. An edge-

colored poset $P$ has the diamond coloring property if whenever subgraph of the Hasse diagram for $P$, then $i=l$ and $j=k$; a necessary and sufficient condition for an edge-colored distributive lattice $L$ to be isomorphic (as an edge-colored poset) to $J_{\text {color }}(P)$ for some vertex-colored poset $P$ is for $L$ to have the diamond coloring property. For $\mathbf{s} \in L$ and $i \in I$, one can see that $\mathbf{c o m p}_{i}(\mathbf{s})$ is the Hasse diagram for a distributive lattice; in particular, $\mathbf{c o m p}_{i}(\mathbf{s})$ is a distributive sublattice of $L$ in the induced order, and a covering relation in $\mathbf{c o m p}_{i}(\mathbf{s})$ is also a covering relation in $L$.

Let $n$ be a positive integer. We use $\mathfrak{g}$ to denote the complex semisimple Lie algebra of 
Figure 2.3: The disjoint sum of the $\beta$-components of the edge-colored lattice $L$ from Figure 2.1.
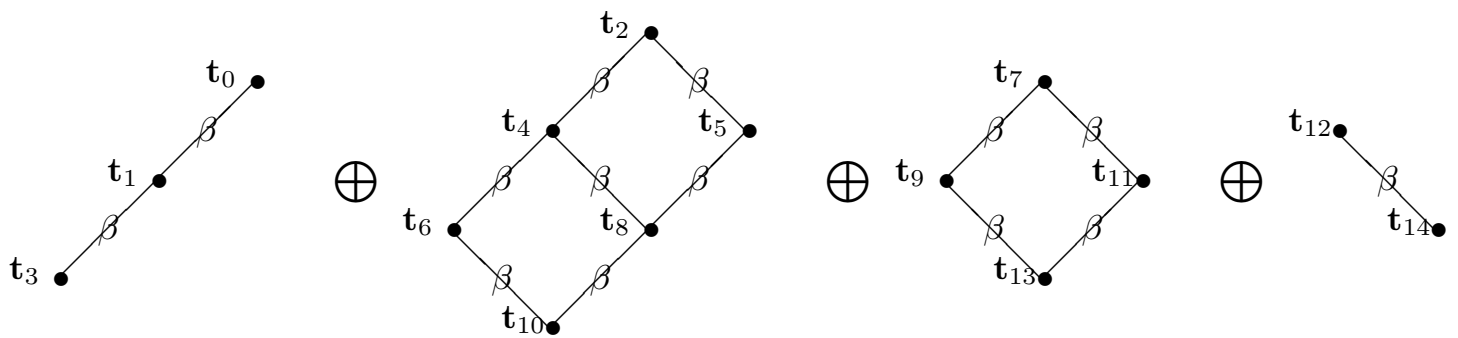

rank $n$ with Chevalley generators $\left\{x_{i}, y_{i}, h_{i}\right\}_{i \in I}$ satisfying the Serre relations associated to a Dynkin diagram $\mathcal{D}$ with $n$ nodes; here the nodes of $\mathcal{D}$ are indexed by a set $I$ of cardinality $n$. We often take $I=\{1, \ldots, n\}$; then our numbering of the nodes of the Dynkin diagrams for the simple Lie algebras follows [Hum] p. 58 with the exception that for us the $B_{n}$ series starts with $n=3$ and the $C_{n}$ series with $n=2$. In what follows the numbers $\mathcal{D}_{i, j}$ and $\mathcal{D}_{j, i}$ can be found in Figure 2.5 by looking at the subgraph of $\mathcal{D}$ determined by the choice of distinct nodes $i$ and $j$; set $\mathcal{D}_{i, i}:=2$ for all $i \in I$. The Cartan matrix for $\mathcal{D}$ (or for $\mathfrak{g}$, when the indexing set $I$ and Dynkin diagram $\mathcal{D}$ are understood) is the matrix $\left(\mathcal{D}_{i, j}\right)_{(i, j) \in I \times I}$. With $i=1$ and $j=2$, the diagrams in Figure 2.5 are Dynkin diagrams for the rank two semisimple Lie algebras $A_{1} \times A_{1}, A_{2}, C_{2}$, and $G_{2}$ respectively $\left(A_{2}, C_{2}\right.$, and $G_{2}$ are simple). Two Dynkin diagrams $\mathcal{D}$ and $\mathcal{D}^{\prime}$ are isomorphic if under some one-to-one correspondence $\sigma: I \longrightarrow I^{\prime}$ of indexing sets it is the case that $\mathcal{D}_{i, j}=\mathcal{D}_{\sigma(i), \sigma(j)}^{\prime}$ and $\mathcal{D}_{j, i}=\mathcal{D}_{\sigma(j), \sigma(i)}^{\prime}$; in this case the mapping which sends $x_{i} \mapsto x_{\sigma(i)}^{\prime}, y_{i} \mapsto y_{\sigma(i)}^{\prime}$, and $h_{i} \mapsto h_{\sigma(i)}^{\prime}$ extends to an isomorphism of the semisimple Lie algebras $\mathfrak{g}$ and $\mathfrak{g}^{\prime}$ with Chevalley generators $\left\{x_{i}, y_{i}, h_{i}\right\}_{i \in I}$ and $\left\{x_{j}^{\prime}, y_{j}^{\prime}, h_{j}^{\prime}\right\}_{j \in I^{\prime}}$ respectively. We use $\left\{\omega_{i}\right\}_{i \in I}$ to denote the fundamental weights corresponding to the nodes of $\mathcal{D}$. The simple root $\alpha_{j}(j \in I)$ can be identified with $\sum_{i \in I} \mathcal{D}_{j, i} \omega_{i}$. We let $\Lambda$ denote the lattice of weights, i.e. the set of all integral linear combinations of the fundamental weights. Elements of $\Lambda$ are called weights. Coordinatize the lattice of weights $\Lambda$ to obtain a one-to-one correspondence with $\mathbb{Z}^{n}$ as follows: identify $\omega_{i}$ with the vector $(0, \ldots, 1, \ldots, 0)$ whose only nonzero coordinate is in the $i$ th position. Then the simple root $\alpha_{j}$ is identified with the $j$ th row vector from the Cartan matrix for $\mathfrak{g}$.

Vector spaces in this paper will be assumed to be complex and finite-dimensional. If $V$ is a $\mathfrak{g}$-module, then there is at least one basis $\mathcal{B}:=\left\{v_{\mathbf{s}}\right\}_{\mathbf{s} \in R}$ (where $R$ is an indexing set with $|R|=\operatorname{dim} V)$ consisting of eigenvectors for the actions of the $h_{i}$ 's: for any $\mathbf{s}$ in $R$ and $i \in I$, there exists an integer $k_{i}(\mathbf{s})$ such that $h_{i} \cdot v_{\mathbf{s}}=k_{i}(\mathbf{s}) v_{\mathbf{s}}$. The weight of the basis vector $v_{\mathbf{s}}$ is the sum $w t\left(v_{\mathbf{s}}\right):=\sum_{i \in I} k_{i}(\mathbf{s}) \omega_{i}$. We say $\mathcal{B}$ is a weight basis for $V$. If $\mu$ is a weight in $\Lambda$, then we let $V_{\mu}$ be the subspace of $V$ spanned by all basis vectors $v_{\mathbf{s}} \in \mathcal{B}$ such that $w t\left(v_{\mathbf{s}}\right)=\mu ; V_{\mu}$ is independent of the choice of weight basis $\mathcal{B}$; any nonzero $v$ in $V_{\mu}$ is said to be a weight vector with weight $\mu$. A nonzero vector $v$ in $V$ is maximal if $x_{i} . v=0$ for every $i \in I$; every weight basis for $V$ will have at least one maximal vector. A $\mathfrak{g}$-module with a unique (up to scalar multiples) maximal vector $v$ has highest weight $\lambda$ 
Figure 2.4: Below is the lattice $L$ from Figure 2.1 recognized as $J_{\text {color }}(P)$. (The vertex-colored poset $P$ is shown in Figure 2.1.

Each order ideal taken from $P$ is identified by the indices of its maximal vertices. For example, $\langle 2,3\rangle$ in $L$ denotes the order ideal $\left\{v_{2}, v_{3}, v_{4}, v_{5}, v_{6}\right\}$ in $P$.)

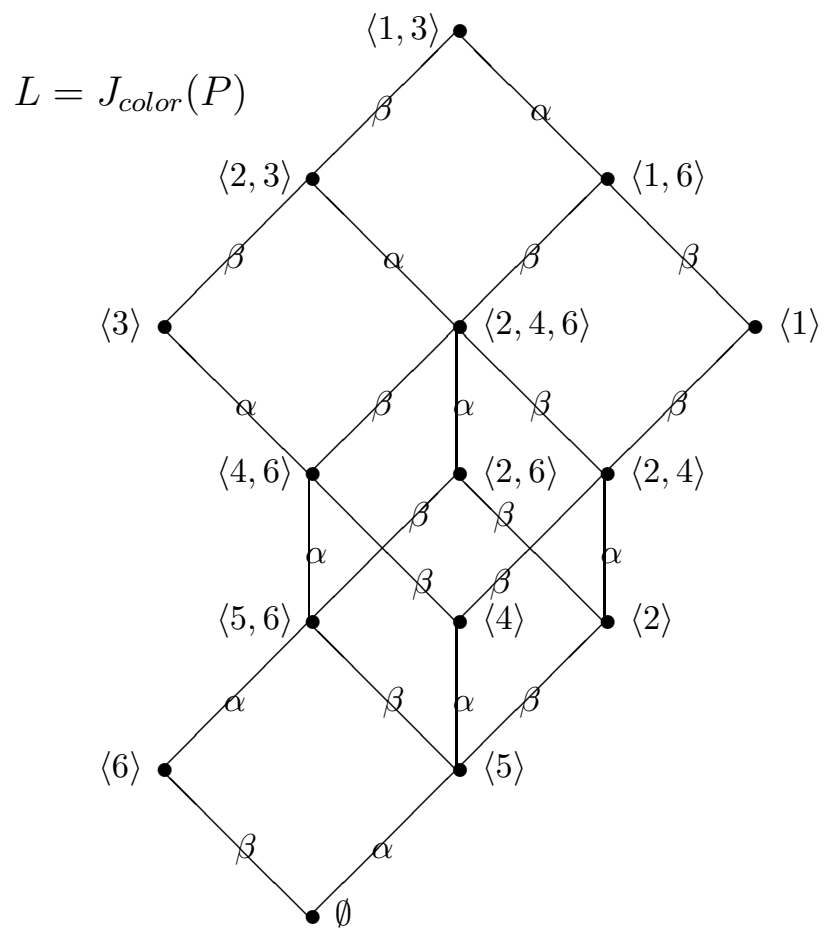

if $v$ has weight $\lambda$; an irreducible module has a unique maximal vector. Finite-dimensional irreducible $\mathfrak{g}$-modules are in one-to-one correspondence with dominant weights, i.e. the nonnegative linear combinations of the fundamental weights: An irreducible $\mathfrak{g}$-module corresponds to the dominant weight $\lambda$ if it has highest weight $\lambda$. The Lie algebra $\mathfrak{g}$ acts on the dual space $V^{*}$ by the rule $(z . f)(v)=-f(z . v)$ for all $v \in V, f \in V^{*}$, and $z \in \mathfrak{g}$.

If a $\mathfrak{g}$-module $V$ has weight basis $\mathcal{B}:=\left\{v_{\mathbf{s}}\right\}_{\mathbf{s} \in R}$, then form an edge-colored directed graph on the vertex set $R$ which indicates the supports of the actions of the generators on the weight basis $\mathcal{B}$ as follows: A directed edge $\mathbf{s} \stackrel{i}{\rightarrow} \mathbf{t}$ of color $i$ is placed from index $\mathbf{s}$ to index $\mathbf{t}$ if $c_{\mathbf{t}, \mathbf{s}} v_{\mathbf{t}}$ (with $c_{\mathbf{t}, \mathbf{s}} \neq 0$ ) appears as a term in the expansion of $x_{i} \cdot v_{\mathbf{s}}$ as a linear combination in the weight basis $\mathcal{B}$, or if $d_{\mathbf{s}, \mathbf{t}} v_{\mathbf{s}}\left(\right.$ with $\left.d_{\mathbf{s}, \mathbf{t}} \neq 0\right)$ appears when we expand $y_{i} \cdot v_{\mathbf{t}}$ in the weight basis $\mathcal{B}$. The resulting edge-colored directed graph, which is also denoted by $R$, is the supporting graph for the weight basis $\mathcal{B}$ of $V$, or simply a supporting graph for $V$. We say an edge-colored directed graph $R$ is a supporting graph for $\mathfrak{g}$ if $R$ is a supporting graph for some representation of $\mathfrak{g}$. Disregarding edge colors, a supporting graph is always the Hasse diagram for a ranked poset (Lemma 3.1.E of [Don1]). To keep track of the actions of the generators on vectors of the weight basis $\mathcal{B}$ we sometimes attach the two coefficients $c_{\mathbf{t}, \mathbf{s}}$ (the " $x$ "-coefficient) and $d_{\mathbf{s}, \mathbf{t}}$ (the " $y$ "-coefficient) to each 
Figure 2.5

\begin{tabular}{|cc|c|c|}
\hline \multicolumn{2}{|c|}{ Subgraph } & $\mathcal{D}_{i, j}$ & $\mathcal{D}_{j, i}$ \\
\hline \hline$i$ & $j$ & 0 & 0 \\
\hline$\bullet$ & $\bullet$ & -1 & -1 \\
\hline$\bullet$ & $\bullet$ & -1 & -2 \\
\hline$i$ & $j$ & -1 & -3 \\
\hline
\end{tabular}

edge $\mathbf{s} \stackrel{i}{\rightarrow} \mathbf{t}$ of $R$. In this case,

$$
x_{i} \cdot v_{\mathbf{s}}=\sum_{\mathbf{t : s} \stackrel{i}{\rightarrow} \mathbf{t}} c_{\mathbf{t}, \mathbf{s}} v_{\mathbf{t}} \quad \text { and } \quad y_{i} \cdot v_{\mathbf{t}}=\sum_{\mathbf{s : s} \stackrel{i}{\rightarrow} \mathbf{t}} d_{\mathbf{s}, \mathbf{t}} v_{\mathbf{s}}
$$

The supporting graph $R$ together with the coefficients $\left\{\left(c_{\mathbf{t}, \mathbf{s}}, d_{\mathbf{s}, \mathbf{t}}\right)\right\}_{\mathbf{s} \rightarrow} \rightarrow \mathbf{t} \in \mathcal{E}(R)$ is the representation diagram (also denoted by $R$ ) for the weight basis $\mathcal{B}$ of $V$. If the coefficients $c_{\mathbf{t}, \mathbf{s}}$ and $d_{\mathbf{s}, \mathbf{t}}$ are positive rational numbers (respectively, positive integers), then we say that the weight basis $\mathcal{B}$ is positive rational (respectively positive integral). A supporting graph $R$ of $V$ is positive rational (resp. positive integral) if there is a positive rational (resp. positive integral) weight basis for $V$ which has $R$ as its supporting graph. We say $R$ is a modular lattice (respectively, distributive lattice) supporting graph if $R$ is a modular lattice (resp. distributive lattice) when viewed as the Hasse diagram for a poset. A supporting graph $R$ for a weight basis $\mathcal{B}$ of $V$ is edge-minimal if no other weight basis for $V$ has its supporting graph appearing as a proper edge-colored subgraph of $R$; the supporting graph $R$ is edgeminimizing if no other weight basis for $V$ has a supporting graph with fewer edges than $R$. In a sense, then, an edge-minimal supporting graph is locally edge-minimizing. Two weight bases $\left\{v_{\mathbf{s}}\right\}_{\mathbf{s} \in R}$ and $\left\{w_{\mathbf{t}}\right\}_{\mathbf{t} \in Q}$ for $V$ are diagonally equivalent if there is an ordering on these bases with respect to which the corresponding change of basis matrix is diagonal; the bases are scalar equivalent if this diagonal matrix is a scalar multiple of the identity. The supporting graph for the weight basis $\mathcal{B}$ is solitary if no weight basis for $V$ has the same supporting graph as $\mathcal{B}$ other than those weight bases that are diagonally equivalent to $\mathcal{B}$. Observe that, up to diagonal equivalence, a representation can have at most a finite number of solitary bases. The adjectives modular (or distributive) lattice, edge-minimal, edge-minimizing, and solitary apply to weight bases as well as supporting graphs. Up to diagonal equivalence, then, a solitary weight basis is uniquely identified by its supporting graph. Figure 5.2 depicts the representation diagram for a weight basis for the "adjoint" representation of $G_{2}$; this basis is positive rational, solitary, and edge-minimal (cf. Theorem 5.1 and Proposition 5.4).

Let $R$ be a ranked poset whose Hasse diagram edges are colored with colors taken from a set $I$ of cardinality $n$. For $i \in I$ and $\mathbf{s}$ in $R$, set $m_{i}(\mathbf{s}):=\rho_{i}(\mathbf{s})-\delta_{i}(\mathbf{s})=2 \rho_{i}(\mathbf{s})-l_{i}(\mathbf{s})$. Let $w t_{R}(\mathbf{s})$ be the $n$-tuple $\left(m_{i}(\mathbf{s})\right)_{i \in I}$. Given a matrix $M=\left(M_{p, q}\right)_{(p, q) \in I \times I}$, then for fixed $i \in I$ let $M^{(i)}$ be the $n$-tuple $\left(M_{i, j}\right)_{j \in I}$, the $i$ th row vector for $M$. We say $R$ satisfies the structure condition for $M$ if $w t_{R}(\mathbf{s})+M^{(i)}=w t_{R}(\mathbf{t})$ whenever $\mathbf{s} \stackrel{i}{\rightarrow} \mathbf{t}$ for some $i \in I$, that is, for all $j \in I$ we have $m_{j}(\mathbf{s})+M_{i, j}=m_{j}(\mathbf{t})$. (In [Don3] it is shown that $M$ must 
in fact be a Cartan matrix if the edge color function is surjective.) Following [DLP1], we say $R$ satisfies the $\mathfrak{g}$-structure condition if $M$ is the Cartan matrix for the Dynkin diagram $\mathcal{D}$ associated to $\mathfrak{g}$. In this case view $w t_{R}: R \longrightarrow \Lambda$ as the function given by $w t_{R}(\mathbf{s})=\sum_{j \in I} m_{j}(\mathbf{s}) \omega_{j}$. Then $R$ satisfies the $\mathfrak{g}$-structure condition if and only if for each simple root $\alpha_{i}$ we have $w t_{R}(\mathbf{s})+\alpha_{i}=w t_{R}(\mathbf{t})$ whenever $\mathbf{s} \stackrel{i}{\rightarrow} \mathbf{t}$ in $R$. (In [Don1] the edges of $R$ in this case were said to "preserve weights.") This condition depends not only on $\mathfrak{g}$ (information from the corresponding Dynkin diagram) but also on the combinatorics of $R$. The edge-colored distributive lattice of Figure 2.6 satisfies the structure condition for the matrix $M=\left(\begin{array}{cc}2 & -1 \\ -1 & 2\end{array}\right)$ and therefore satisfies the $A_{2}$-structure condition. The following simple lemma merely observes when the matrix $M$ is uniquely determined by an edge-colored ranked poset $R$ that satisfies some structure condition.

Figure 2.6: For each element $\mathbf{t}$ of the lattice $L$ from Figure 2.1, we compute $w t_{L}(\mathbf{t})=\left(m_{\alpha}(\mathbf{t}), m_{\beta}(\mathbf{t})\right)$.

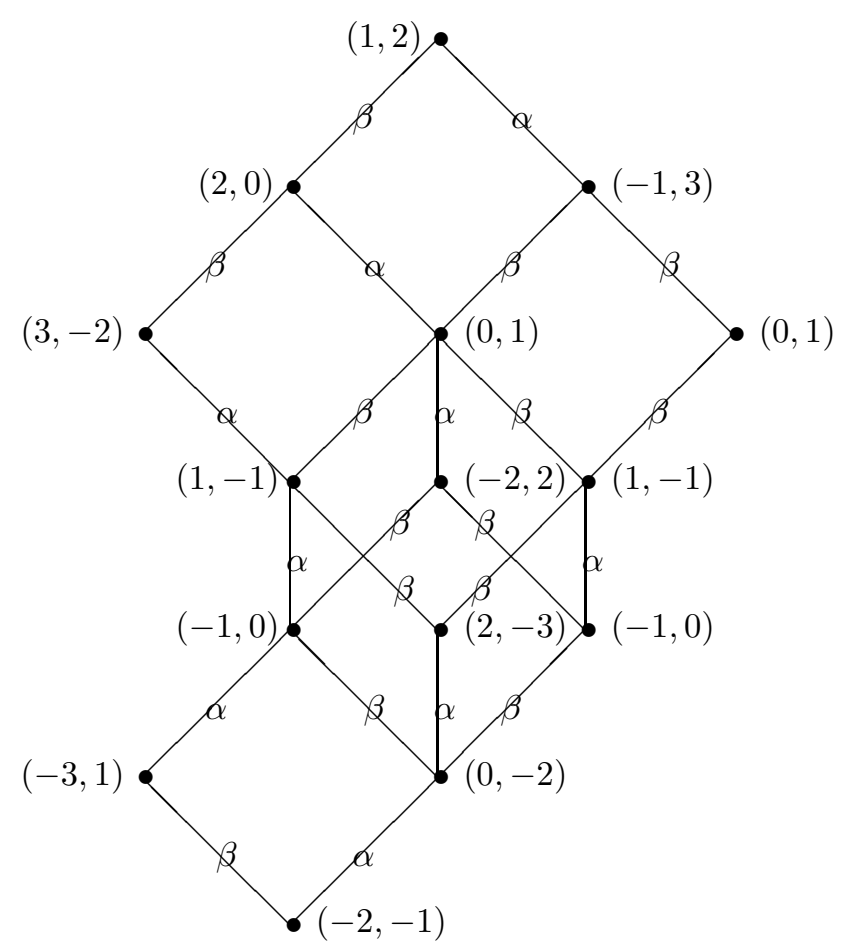

Lemma 2.1 Let $R$ be a ranked poset with edges colored by a set $I$. Suppose the edge coloring function edgecolor en $_{R}: \mathcal{E}(R) \longrightarrow I$ is surjective. (1) Suppose $R$ satisfies the structure condition for matrices $M=\left(M_{i, j}\right)_{(i, j) \in I \times I}$ and $M^{\prime}=\left(M_{i, j}^{\prime}\right)_{(i, j) \in I \times I}$. Then for all $i, j \in I, M_{i, j}=M_{i, j}^{\prime}$, and this quantity is an integer. Moreover, $M_{i, i}=2$ for all $i \in I$. (2) Let $\mathcal{D}$ and $\mathcal{D}^{\prime}$ be two Dynkin diagrams whose nodes are indexed by $I$, and let $\mathfrak{g}$ and $\mathfrak{g}^{\prime}$ be the corresponding semisimple Lie algebras. If $R$ satisfies the $\mathfrak{g}$-structure and $\mathfrak{g}^{\prime}$-structure conditions, then $\mathcal{D}$ and $\mathcal{D}^{\prime}$ are isomorphic under the correspondence given by $I$, and hence $\mathfrak{g} \cong \mathfrak{g}^{\prime}$ 
Proof. (2) follows from (1). For (1), note that for each $j \in I$ there is an edge $\mathbf{s} \stackrel{j}{\rightarrow} \mathbf{t}$ in $R$. Then for each $i \in I, M_{j, i}=m_{i}(\mathbf{t})-m_{i}(\mathbf{s})=M_{j, i}^{\prime}$. When $i=j$, note that $\rho_{i}(\mathbf{t})=\rho_{i}(\mathbf{s})+1$ and $l_{i}(\mathbf{s})=l_{i}(\mathbf{t})$, so $2=m_{i}(\mathbf{t})-m_{i}(\mathbf{s})$.

An edge-labelled poset $R$ with colors from $I$ is an edge-colored ranked poset $R$ with edge colors from the set $I$ together with an assignment of edge coefficients $\left\{\left(c_{\mathbf{t}, \mathbf{s}}, d_{\mathbf{s}, \mathbf{t}}\right)\right\}_{\mathbf{s} \rightarrow i} \rightarrow \mathbf{t} \in \mathcal{E}(R)$. In the ordered pair $\left(c_{\mathbf{t}, \mathbf{s}}, d_{\mathbf{s}, \mathbf{t}}\right)$, we think of $c_{\mathbf{t}, \mathbf{s}}$ as an $x$-coefficient and $d_{\mathbf{s}, \mathbf{t}}$ as a $y$-coefficient. We call $\pi_{\mathbf{s}, \mathbf{t}}:=c_{\mathbf{t}, \mathbf{s}} d_{\mathbf{s}, \mathbf{t}}$ the edge product associated to a given edge $\mathbf{s} \stackrel{i}{\rightarrow} \mathbf{t}$ in the edge-labelled poset $R$. If $\sigma: I \rightarrow I^{\prime}$ is a mapping of sets, then we regard the recoloring $R^{\sigma}$ of $R$ to be an edge-labelled poset where the coefficients assigned to an edge $\mathbf{s} \stackrel{\sigma(i)}{\longrightarrow} \mathbf{t}$ in $R^{\sigma}$ are the same as the coefficients assigned to the edge $\mathbf{s} \stackrel{i}{\rightarrow} \mathbf{t}$ in $R$. Make the edge-colored poset $R^{*}$ an edge-labelled poset as follows: Give an edge $\mathbf{t}^{*} \stackrel{i}{\rightarrow} \mathbf{s}^{*}$ in $R^{*}$ coefficients $c_{\mathbf{s}^{*}, \mathbf{t}^{*}}:=d_{\mathbf{s}, \mathbf{t}}$ and $d_{\mathbf{t}^{*}, \mathbf{s}^{*}}:=c_{\mathbf{t}, \mathbf{s}}$, where the edge $\mathbf{s} \stackrel{i}{\rightarrow} \mathbf{t}$ in $R$ has $x$ - and $y$-coefficients $c_{\mathbf{t}, \mathbf{s}}$ and $d_{\mathbf{s}, \mathbf{t}}$ respectively.

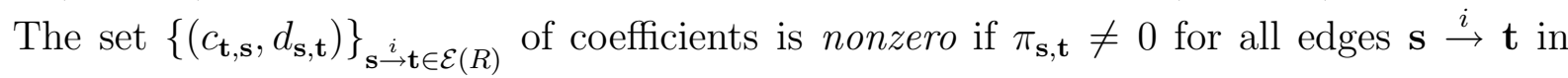
$\mathcal{E}(R)$. The edge-labelled poset $R$ satisfies the crossing condition if for any $\mathbf{s}$ in $R$ and any color $i \in I$, we have

$$
\sum_{\mathbf{r}: \mathbf{r} \stackrel{i}{\rightarrow} \mathbf{s}} \pi_{\mathbf{r}, \mathbf{s}}-\sum_{\substack{\mathbf{t}: \mathbf{s} \\ \rightarrow}} \pi_{\mathbf{s}, \mathbf{t}}=m_{i}(\mathbf{s}) .
$$

A relation of form (2) is a crossing relation. The edge-labelled poset $R$ satisfies the diamond condition if for any pair of vertices $\mathbf{s}$ and $\mathbf{t}$ in $R$ and any colors $i$ and $j$ in $I$, we have

$$
\sum_{\mathbf{u}: \mathbf{s} \stackrel{j}{\rightarrow} \mathbf{u} \text { and } \mathbf{t} \stackrel{i}{\rightarrow} \mathbf{u}} c_{\mathbf{u}, \mathbf{s}} d_{\mathbf{t}, \mathbf{u}}=\sum_{\mathbf{r}: \mathbf{r} \stackrel{i}{\rightarrow} \mathbf{s} \text { and } \mathbf{r} \stackrel{j}{\rightarrow} \mathbf{t}} d_{\mathbf{r}, \mathbf{s}} c_{\mathbf{t}, \mathbf{r}},
$$

where an empty sum is zero. Suppose there is a unique element $\mathbf{u}$ such that $\mathbf{s} \stackrel{j}{\rightarrow} \mathbf{u}$ and $\mathbf{t} \stackrel{i}{\rightarrow} \mathbf{u}$, and suppose there is a unique element $\mathbf{r}$ such that $\mathbf{r} \stackrel{i}{\rightarrow} \mathbf{s}$ and $\mathbf{r} \stackrel{j}{\rightarrow} \mathbf{t}$. Then we

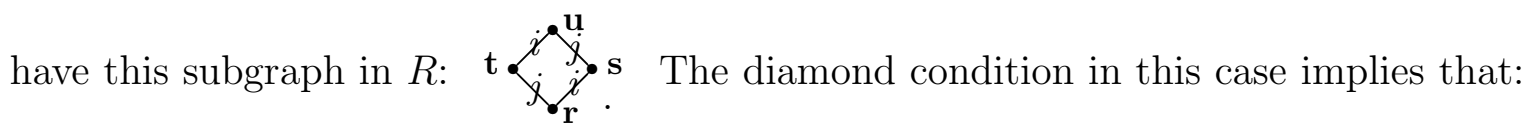

$$
c_{\mathbf{u}, \mathbf{s}} d_{\mathbf{t}, \mathbf{u}}=d_{\mathbf{r}, \mathbf{s}} c_{\mathbf{t}, \mathbf{r}} \quad \text { and } \quad c_{\mathbf{u}, \mathbf{t}} d_{\mathbf{s}, \mathbf{u}}=d_{\mathbf{r}, \mathbf{t}} c_{\mathbf{s}, \mathbf{r}}
$$

and

$$
\pi_{\mathbf{s}, \mathbf{u}} \pi_{\mathbf{t}, \mathbf{u}}=\pi_{\mathbf{r}, \mathbf{s}} \pi_{\mathbf{r}, \mathbf{t}}
$$

Any relation of the form (3), (4), or (5) is a diamond relation. We let $V[R]$ be the complex vector space with basis $\left\{v_{\mathbf{s}}\right\}_{\mathbf{s} \in R}$, and for $i \in I$, we let $x_{i}$ and $y_{i}$ act on $V[R]$ using the identities at (1) above. The following proposition is a reformulation of Proposition 3.4 of [Don1]. As an example, one can apply this result to the edge-labelled poset of Figure 5.2 to see that it is a representation diagram for some representation of $G_{2}$.

Proposition 2.2 Let $\mathcal{D}$ be a Dynkin diagram whose nodes are indexed by a set $I$, and let $\mathfrak{g}$ be the associated semisimple Lie algebra with Chevalley generators $\left\{x_{i}, y_{i}, h_{i}\right\}_{i \in I}$. Let $R$ be an edge-labelled poset with colors from $I$ having the property that at least one 
of the two coefficients $\left(c_{\mathbf{t}, \mathbf{s}}\right.$ or $\left.d_{\mathbf{s}, \mathbf{t}}\right)$ assigned to any given edge $\mathbf{s} \stackrel{i}{\rightarrow} \mathbf{t}$ in $P$ is nonzero. Then $V[R]$ is a $\mathfrak{g}$-module (with the action of $\mathfrak{g}$ induced by the actions on $V[R]$ of the $x_{i}$ 's and $y_{i}$ 's as described at (1) above) and the edge-labelled poset $R$ is a representation diagram for the weight basis $\left\{v_{\mathbf{s}}\right\}_{\mathbf{s} \in R}$ of $V[R]$ if and only if $R$ satisfies the diamond, crossing, and $\mathfrak{g}$-structure conditions. In this case, $h_{i} \cdot v_{\mathbf{s}}=m_{i}(\mathbf{s}) v_{\mathbf{s}}$ for any $\mathbf{s}$ in $R$ and $i \in I$, so $w t\left(v_{\mathbf{s}}\right)=\sum_{i \in I} m_{i}(\mathbf{s}) \omega_{i}=w t_{R}(\mathbf{s})$.

Lemma 2.3 Suppose $R$ is the representation diagram for some weight basis of a $\mathfrak{g}$-module $V$. Then the edge-labelled poset $R^{*}$ is a representation diagram for the dual representation $V^{*}$ of $\mathfrak{g}$. Moreover, the edge-colored poset $R^{*}$ is a positive rational (respectively positive integral, modular (or distributive) lattice, solitary, edge-minimal, edge-minimizing) supporting graph for $V^{*}$ if $R$ is positive rational (respectively positive integral, modular (or distributive) lattice, solitary, edge-minimal, edge-minimizing).

Proof. It is easy to see that the edge-labelled poset $R^{*}$ satisfies the diamond, crossing, and $\mathfrak{g}$-structure conditions, and hence by Proposition 2.2 it is a representation diagram for some representation of $\mathfrak{g}$. By Lemma 3.3.A of [Don1], this representation is isomorphic to $V^{*}$. Clearly the edge coefficients attached to $R^{*}$ are all positive rational (respectively positive integral) if the coefficients for $R$ are positive rational (resp. positive integral). The poset-dual of a modular (or distributive) lattice is also a modular (or distributive) lattice.

The remaining claims of the lemma can be proved by contrapositive. This is effected by the following observation: The poset $\left(R^{*}\right)^{*}$ is isomorphic to $R$ as an edge-colored poset via the correspondence of vertices $\mathbf{x}^{* *} \mapsto \mathbf{x}$; then corresponding edges of the edgelabelled posets $R$ and $\left(R^{*}\right)^{*}$ have identical edge coefficients. So suppose $R^{*}$ contains as a proper edge-colored subposet some supporting graph $Q^{*}$ for $V^{*}$ where $Q$ is a proper edge-colored subposet of $R$. Then $Q$ is a supporting graph for $V \cong\left(V^{*}\right)^{*}$ since $\left(Q^{*}\right)^{*}$ is a supporting graph for $\left(V^{*}\right)^{*}$ and $Q \cong\left(Q^{*}\right)^{*}$. Thus $R$ contains as a proper edgecolored subposet a supporting graph $Q$ for $V$. That is, if $R^{*}$ is not edge-minimal, then $R$ is not edge-minimal. It is similarly easy to show that if $R^{*}$ is not edge-minimizing, then $R$ is not edge-minimizing. Now suppose that $R^{*}$ is not solitary as a supporting graph for $V^{*}$. Then if $\left\{v_{\mathbf{t}^{*}}\right\}_{\mathbf{t}^{*} \in R^{*}}$ denotes a weight basis for $V^{*}$ whose representation diagram is the edge-labelled poset $R^{*}$, there must be another weight basis $\left\{w_{\mathbf{t}^{*}}\right\}_{\mathbf{t}^{*} \in R^{*}}$ for $V^{*}$ with supporting graph $R^{*}$ and such that $\left\{v_{\mathbf{t}^{*}}\right\}$ and $\left\{w_{\mathbf{t}^{*}}\right\}$ are not diagonally equivalent. Write $w_{\mathbf{t}^{*}}=\sum_{\mathbf{s}^{*} \in R^{*}} a_{\mathbf{s}^{*}, \mathbf{t}^{*}} v_{\mathbf{s}^{*}}$, so that the scalars $\left(a_{\mathbf{s}^{*}, \mathbf{t}^{*}}\right)_{\left(\mathbf{s}^{*}, \mathbf{t}^{*}\right) \in R^{*} \times R^{*}}$ describe the change of basis. Now let $\left\{u_{\mathbf{t}}\right\}_{\mathbf{t} \in R}$ be a weight basis for $V$ with representation diagram $R$. Set $z_{\mathbf{t}}:=\sum_{\mathbf{s} \in R} a_{\mathbf{s}^{*}, \mathbf{t}^{*}} u_{\mathbf{t}}$. Then $\left\{z_{\mathbf{t}}\right\}_{\mathbf{t} \in R}$ is a weight basis for $V$ that is not diagonally equivalent to $\left\{u_{\mathbf{t}}\right\}_{\mathbf{t} \in R}$ but has supporting graph $R$.

The following (obvious) lemma follows from the definitions but is useful as a principle, particularly when the Dynkin diagram has symmetry $\left(A_{n}, D_{n}\right.$, and $\left.E_{6}\right)$ or when other numberings of the Dynkin diagram are convenient.

Lemma 2.4 Let $\mathcal{D}$ and $\mathcal{D}^{\prime}$ be Dynkin diagrams with nodes indexed by $I$ and $I^{\prime}$ respectively such that $\mathcal{D}$ and $\mathcal{D}^{\prime}$ are isomorphic under a one-to-one correspondence $\sigma: I \longrightarrow I^{\prime}$. 
Let $\mathfrak{g}$ and $\mathfrak{g}^{\prime}$ be the respective semisimple Lie algebras. Let $R$ be a ranked poset with edges colored by the set $I$, and consider the recoloring $R^{\sigma}$. Then $R$ is a supporting graph for $\mathfrak{g}$ (respectively, positive integral, positive rational, modular (or distributive) lattice, solitary, edge-minimal, or edge-minimizing support) if and only if $R^{\sigma}$ is a supporting graph for $\mathfrak{g}^{\prime}$ (respectively, positive integral, positive rational, modular (or distributive) lattice, solitary, edge-minimal, or edge-minimizing support).

Lemmas 2.3 and 2.4 can work in tandem as follows. For any Dynkin diagram $\mathcal{D}$ there is a special permutation $\sigma_{0}$ of the index set $I$ that yields a symmetry of the Dynkin diagram. See the discussion in Section 2 of [ADLMPW]. For $A_{2}$ with index set $\{1,2\}$, we have $\sigma_{0}(1)=2$ and $\sigma_{0}(2)=1$; for $A_{1} \times A_{1}, C_{2}$, and $G_{2}, \sigma_{0}$ is trivial. Here we extend the notion of the " $\triangle$ " operation from [ADLMPW] on edge-colored and vertex-colored posets to an operation on edge-labelled posets as follows: Given a representation diagram $R$ for a representation $V$ of $\mathfrak{g}$, we let $R^{\triangle}$ be the edge-labelled poset $\left(R^{*}\right)^{\sigma_{0}}$ and call $R^{\triangle}$ the $\sigma_{0}$-recolored dual of $R$. That is, take the edge-labelled poset $R^{*}$ and recolor the edges by applying $\sigma_{0}$ as in Lemma 2.4 to obtain $\left(R^{*}\right)^{\sigma_{0}}$. Observe that $\left(R^{\triangle}\right)^{\triangle}=R$. For an example, see Figure 2.7.

Figure 2.7: $L^{\triangle}$ for the lattice $L$ from Figure 2.1.

(In Theorem 5.1 we see that the edge-colored lattice $L$ is a supporting graph for $A_{2}$ with Dynkin diagram $\stackrel{\alpha}{\alpha} \stackrel{\beta}{\bullet}$.)

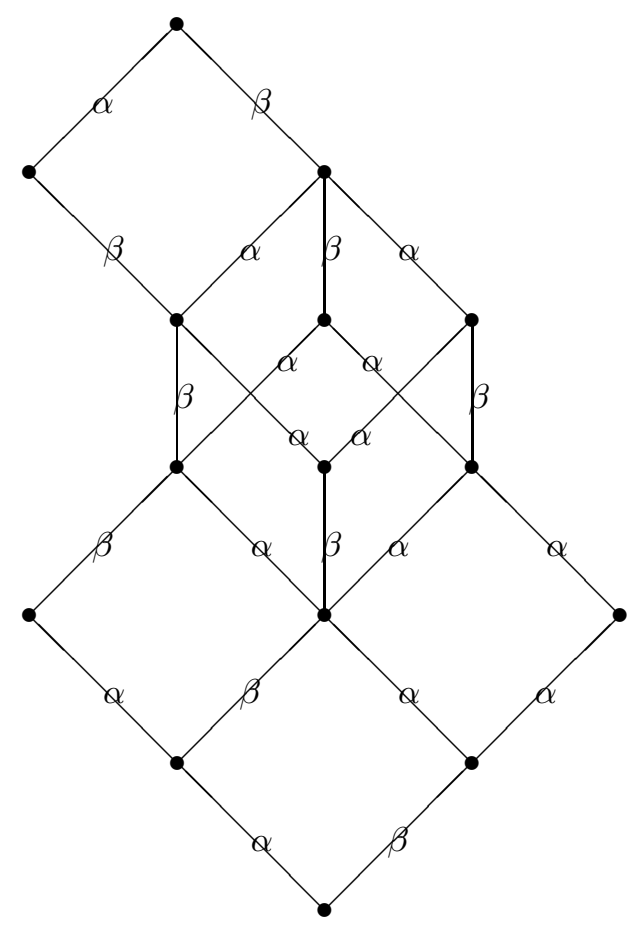

Proposition 2.5 In the notation of the preceding paragraph, the edge-labelled poset $R^{\triangle}$ is also a representation diagram for the $\mathfrak{g}$-module $V$. Moreover, the edge-colored poset $R^{\triangle}$ is a positive rational (respectively positive integral, modular (or distributive) lattice, 
solitary, edge-minimal, edge-minimizing) supporting graph for $V$ if $R$ is positive rational (respectively positive integral, modular (or distributive) lattice, solitary, edge-minimal, edge-minimizing).

Proof. The only assertion that does not immediately follow from Lemmas 2.3 and 2.4 is that $R^{\triangle}$ is a supporting graph for the $\mathfrak{g}$-module $V$. But this follows from Lemma 2.2 of $[$ ADLMPW].

\section{Grid posets; two-color grid posets; semistandard posets and lattices}

Following [ADLMPW], given a finite poset $\left(P, \leq_{P}\right)$, a chain function for $P$ is a function chain $: P \longrightarrow\{1,2, \ldots, m\}$ for some positive integer $m$ such that $(1) \mathcal{C}_{i}:=\operatorname{chain}^{-1}(i)$ is a (possibly empty) chain in $P$ for $1 \leq i \leq m$, and (2) given any covering relation $u \rightarrow v$ in $P$, it is the case that either $\operatorname{chain}(u)=\operatorname{chain}(v)$ or chain $(u)=\operatorname{chain}(v)+1$. A grid poset is a finite poset $\left(P, \leq_{P}\right)$ together with a chain function chain $: P \longrightarrow\{1,2, \ldots, m\}$ for some positive integer $m$. We let $\mathcal{I}_{P}$ be the totally ordered set whose elements are the elements of $P$ and whose ordering is given by the following rule: for distinct $u$ and $v$ in $P$ write $u<_{T_{P}} v$ if and only if (1) chain $(u)<\operatorname{chain}(v)$ or $(2)$ chain $(u)=\operatorname{chain}(v)$ with $v<_{P} u$. Let $l:=|P|$. Number the vertices of $P v_{1}, v_{2}, \ldots, v_{l}$ so that $v_{p}<_{T_{P}} v_{q}$ whenever $1 \leq p<q \leq l$. Let $L:=J(P)$ be the distributive lattice of order ideals taken from $P$. We simultaneously think of order ideals taken from $P$ as subsets of $P$ and as elements of $L$. Let $\mathbf{m}$ be the maximal element of $L$ (so as sets, $\mathbf{m}=P$ ). For $1 \leq i \leq l$, set $\mathbf{b}_{i}:=P \backslash\left\{v_{1}, \ldots, v_{i}\right\}$, and set $\mathbf{b}_{0}:=\mathbf{m}$; observe that each $\mathbf{b}_{i}$ is an order ideal taken from $P$. The sequence of order ideals $\left(\mathbf{b}_{0}, \mathbf{b}_{1}, \cdots, \mathbf{b}_{l}\right)$ is the boundary of $L$. Let $\rho: L \longrightarrow\{0, \ldots, l\}$ denote the rank function of $L$. Then $\mathbf{b}_{i}$ is the unique boundary element in the set $\rho^{-1}(l-i)$. If $\mathbf{s} \rightarrow \mathbf{t}$ is an edge in $L$, then necessarily $\mathbf{t} \backslash \mathbf{s}=\{v\}$ for some $v$ in $P$. Associate to $L$ the following ancestor function (cf. [DLP2]): ancestor $_{L}: L \backslash\{\mathbf{m}\} \longrightarrow L$ is given by the rule ancestor $_{L}(\mathbf{s})=\mathbf{s} \cup\left\{v_{p}\right\}$ where $v_{p}$ is the largest element in $\mathcal{T}_{P}$ such that $v_{p} \notin \mathbf{s}$ and $\mathbf{s} \cup\left\{v_{p}\right\} \in L$. We assign to any given element $\mathbf{s}$ in $L$ the coordinates $\operatorname{coord}(\mathbf{s}):=\left(s_{1}, \ldots, s_{m}\right)$, where $s_{i}$ is $\left|\mathcal{C}_{i} \cap \mathbf{s}\right|$. For $1 \leq i \leq m$, let $c_{i}:=\left|\mathcal{C}_{i}\right|$; then $\operatorname{coord}(\mathbf{m})=\left(c_{1}, \ldots, c_{m}\right)$. If $\mathbf{s}$ is a descendant of $\mathbf{t}$ in $L$ where $\mathbf{t}$ has coordinates $\operatorname{coord}(\mathbf{t})=\left(t_{1}, \ldots, t_{m}\right)$, then for some $i$ with $1 \leq i \leq m$ we have $\operatorname{coord}(\mathbf{s})=\left(t_{1}, \ldots, t_{i-1}, t_{i}-1, t_{i+1}, \ldots, t_{m}\right)$; we use the notation $\mathbf{t}^{(i)}$ to refer to this particular descendant of $\mathbf{t}$. Define a total ordering $\mathcal{T}_{L}$ on the elements of $L$ as follows: for distinct $\mathbf{s}$ and $\mathbf{t}$ in $L$, write $\mathbf{s}<_{\mathcal{T}_{L}} \mathbf{t}$ if and only if (1) $\rho(\mathbf{s})>\rho(\mathbf{t})$; or $(2)$ $\rho(\mathbf{s})=\rho(\mathbf{t})$ and $\operatorname{dist}(\mathbf{s}, \mathbf{b})<\operatorname{dist}(\mathbf{t}, \mathbf{b})$, where $\mathbf{b}$ is the unique boundary element of $L$ for which $\rho(\mathbf{b})=\rho(\mathbf{s})=\rho(\mathbf{t})$; or $(3) \rho(\mathbf{s})=\rho(\mathbf{t}), \operatorname{dist}(\mathbf{s}, \mathbf{b})=\operatorname{dist}(\mathbf{t}, \mathbf{b})$, and there exists a $j$ such that $s_{j}>t_{j}$ while $s_{i}=t_{i}$ for $i>j$.

Lemma 3.1 Let $P$ be a grid poset as above, and let $\mathbf{s}$ and $\mathbf{t}$ be elements of $L=J(P)$ with $\operatorname{coord}(\mathbf{s})=\left(s_{1}, \ldots, s_{m}\right)$ and $\operatorname{coord}(\mathbf{t})=\left(t_{1}, \ldots, t_{m}\right)$. Then:

$\begin{aligned} & \text { (1) } \operatorname{coord}(\mathbf{s} \vee \mathbf{t})=\left(\max \left(s_{1}, t_{1}\right), \ldots, \max \left(s_{m}, t_{m}\right)\right) \\ & \text { and } \quad \operatorname{coord}(\mathbf{s} \wedge \mathbf{t})=\left(\min \left(s_{1}, t_{1}\right), \ldots, \min \left(s_{m}, t_{m}\right)\right) .\end{aligned}$ 
(2) $\operatorname{dist}(\mathbf{s}, \mathbf{t})=\sum_{i=1}^{m}\left|s_{i}-t_{i}\right|$.

(3) Suppose $\mathbf{t}^{(i)}$ and $\mathbf{t}^{(j)}$ are descendants of $\mathbf{t}$ in $L$ with $i<j$. Let $\mathbf{b}$ be the unique boundary element with the same rank as $\mathbf{t}^{(i)}$ and $\mathbf{t}^{(j)} . T$ Then $\operatorname{dist}\left(\mathbf{t}^{(i)}, \mathbf{b}\right) \leq \operatorname{dist}\left(\mathbf{t}^{(j)}, \mathbf{b}\right)$ and $\mathbf{t}^{(i)}<_{\mathcal{T}_{L}} \mathbf{t}^{(j)}$.

(4) Suppose $\mathbf{s} \rightarrow \mathbf{t}$ in $L$, and let $\mathbf{t}^{\prime}:=\operatorname{ancestor}_{L}(\mathbf{s})$. If $\mathbf{t}^{\prime} \neq \mathbf{t}$, then $\mathbf{t}^{\prime}<_{\mathcal{T}_{L}} \mathbf{t}$.

Proof. Part (1) follows immediately from the definitions. For part (2), first observe that the rank of $\mathbf{s}$ in $L$ is $\rho(\mathbf{s})=\sum_{i=1}^{m} s_{i}$. Now apply part (1) together with the fact that $\operatorname{dist}(\mathbf{s}, \mathbf{t})=2 \rho(\mathbf{s} \vee \mathbf{t})-\rho(\mathbf{s})-\rho(\mathbf{t})=\rho(\mathbf{s})+\rho(\mathbf{t})-2 \rho(\mathbf{s} \wedge \mathbf{t})$. For part (3), it suffices to show that $\operatorname{dist}\left(\mathbf{t}^{(i)}, \mathbf{b}\right) \leq \operatorname{dist}\left(\mathbf{t}^{(j)}, \mathbf{b}\right)$. Write $\operatorname{coord}\left(\mathbf{t}^{(k)}\right)=\left(t_{1}^{(k)}, \ldots, t_{m}^{(k)}\right)$ if $k$ is $i$ or $j$, and write $\operatorname{coord}(\mathbf{b})=\left(0, \ldots, 0, b_{p}, c_{p+1}, \ldots, c_{m}\right)$. Then $t_{i}^{(i)}=t_{i}-1, t_{j}^{(i)}=t_{j}, t_{i}^{(j)}=t_{i}$, and $t_{j}^{(j)}=t_{j}-1$. From (2) we have $\operatorname{dist}\left(\mathbf{t}^{(i)}, \mathbf{b}\right)=t_{1}^{(i)}+\cdots+t_{p-1}^{(i)}+\left|b_{p}-t_{p}^{(i)}\right|+\left(c_{p+1}-t_{p+1}^{(i)}\right)+$ $\cdots+\left(c_{m}-t_{m}^{(i)}\right)$ and a similar expression for $\operatorname{dist}\left(\mathbf{t}^{(j)}, \mathbf{b}\right) . \operatorname{Then} \operatorname{dist}\left(\mathbf{t}^{(j)}, \mathbf{b}\right)-\operatorname{dist}\left(\mathbf{t}^{(i)}, \mathbf{b}\right)$

$$
\begin{aligned}
= & \left\{\begin{array}{lr}
t_{i}^{(j)}-t_{i}^{(i)}+t_{j}^{(j)}-t_{j}^{(i)} & \text { if } i<j<p \\
c_{i}-t_{i}^{(j)}-\left(c_{i}-t_{i}^{(i)}\right)+c_{j}-t_{j}^{(j)}-\left(c_{j}-t_{j}^{(i)}\right) & \text { if } p<i<j \\
t_{i}^{(j)}-t_{i}^{(i)}+c_{j}-t_{j}^{(j)}-\left(c_{j}-t_{j}^{(i)}\right) & \text { if } i<p<j \\
t_{i}^{(j)}-t_{i}^{(i)}+\left|b_{p}-t_{p}^{(j)}\right|-\left|b_{p}-t_{p}^{(i)}\right| & \text { if } i<p, j=p \\
\left|b_{p}-t_{p}^{(j)}\right|-\left|b_{p}-t_{p}^{(i)}\right|+c_{j}-t_{j}^{(j)}-\left(c_{j}-t_{j}^{(i)}\right) & \text { if } i=p, p<j
\end{array}\right. \\
= & \left\{\begin{array}{lr}
t_{i}-\left(t_{i}-1\right)+t_{j}-1-t_{j}=0 & \text { if } i<j<p \\
c_{i}-t_{i}-\left(c_{i}-\left(t_{i}-1\right)\right)+c_{j}-\left(t_{j}-1\right)-\left(c_{j}-t_{j}\right)=0 & \text { if } p<i<j \\
t_{i}-\left(t_{i}-1\right)+c_{j}-\left(t_{j}-1\right)-\left(c_{j}-t_{j}\right)=2 & \text { if } i<p<j \\
t_{i}-\left(t_{i}-1\right)+\left|b_{p}-\left(t_{p}-1\right)\right|-\left|b_{p}-t_{p}\right|=0 \text { or } 2 & \text { if } i<p, j=p \\
\left|b_{p}-t_{p}\right|-\left|b_{p}-\left(t_{p}-1\right)\right|+c_{j}-\left(t_{j}-1\right)-\left(c_{j}-t_{j}\right)=0 \text { or } 2 & \text { if } i=p, p<j
\end{array}\right.
\end{aligned}
$$

For $(4)$, note that for some $1 \leq p \leq m, \operatorname{coord}(\mathbf{t})=\left(s_{1}, \ldots, s_{p-1}, s_{p}+1, s_{p+1}, \ldots, s_{m}\right)$. Moreover, we have $\operatorname{coord}\left(\mathbf{t}^{\prime}\right)=\left(s_{1}, \ldots, s_{q-1}, s_{q}+1, s_{q+1}, \ldots, s_{m}\right)$ for some $q \neq p$ since $\mathbf{t}^{\prime} \neq \mathbf{t}$. By definition of ancestor $_{L}$, it follows that $q>p$. Let $\mathbf{u}$ be the least upper bound of $\mathbf{t}$ and $\mathbf{t}^{\prime}$ in $L$. Then $\operatorname{coord}(\mathbf{u})=\left(s_{1}, \ldots, s_{p}+1, \ldots, s_{q}+1, \ldots, s_{m}\right)$. When we view the descendants $\mathbf{t}^{\prime}=\mathbf{u}^{(p)}$ and $\mathbf{t}=\mathbf{u}^{(q)}$ of $\mathbf{u}$ in the light of part(3), then we see that $\mathbf{t}^{\prime}<_{\mathcal{T}_{L}} \mathbf{t}$.

A two-color function for a grid poset $\left(P, \leq_{P}\right.$, chain :P $\left.P\{1,2, \ldots, m\}\right)$ is a function color $: P \longrightarrow \Delta$ such that $(1)|\Delta|=2,(2) \operatorname{color}(u)=\operatorname{color}(v)$ if $\operatorname{chain}(u)=\operatorname{chain}(v)$, and (3) if $u$ and $v$ are in the same connected component of $P$ with chain $(u)=\operatorname{chain}(v)+1$, then $\operatorname{color}(u) \neq \operatorname{color}(v)$. A two-color grid poset is a grid poset $\left(P, \leq_{P}\right.$, chain :P $\longrightarrow$ $\{1, \ldots, m\})$ together with a two-color function color $: P \longrightarrow \Delta$. A two-color grid poset should be thought of as a certain kind of vertex-colored poset. We will associate to a two-color grid poset $P$ the edge-colored distributive lattice $L:=J_{\text {color }}(P)$. We say a two-color grid poset $P$ has the max property if $P$ is isomorphic to a two-color grid poset $\left(Q, \leq_{Q}\right.$, chain $: Q \longrightarrow\{1,2, \ldots, m\}$, color $\left.: Q \longrightarrow \Delta\right)$ with a surjective chain function 
such that (1) if $u$ is any maximal element in the poset $Q$, then chain $(u) \leq 2$, and (2) if $v \neq u$ is another maximal element in $Q$, then $\operatorname{color}(u) \neq \operatorname{color}(v)$. We will often take $\Delta:=\{\alpha, \beta\}$. When we switch (or reverse) the vertex colors of $P$ we replace the color function color $: P \longrightarrow\{\alpha, \beta\}$ with the color function color ${ }^{\prime}: P \longrightarrow\{\alpha, \beta\}$ given by: $\operatorname{color}^{\prime}(v)=\alpha$ if $\operatorname{color}(v)=\beta$, and $\operatorname{color}^{\prime}(v)=\beta$ if $\operatorname{color}(v)=\alpha$. Similarly, one can switch (or reverse) the edge colors of $L$. In Figures 3.3 and 3.4 we depict eight two-color grid posets with the max property; the numbering of the vertices for each poset $P$ follows the total ordering $\mathcal{T}_{P}$. The vertex-colored poset $P$ of Figure 2.1 is a two-color grid poset with the max property. The lattice $L$ in that figure is $J_{\text {color }}(P)$; the total ordering $\mathcal{T}_{L}$ is indicated by the indices of the elements of $L$ so that $\mathbf{t}_{0}<_{\mathcal{T}_{L}} \mathbf{t}_{1}<_{\mathcal{T}_{L}} \cdots<_{\mathcal{T}_{L}}<\mathbf{t}_{14}$. The boundary is the sequence $\left(\mathbf{t}_{0}, \mathbf{t}_{1}, \mathbf{t}_{3}, \mathbf{t}_{6}, \mathbf{t}_{9}, \mathbf{t}_{12}, \mathbf{t}_{14}\right)$. The order ideals corresponding to elements of the lattice are depicted in Figure 2.4.

Lemma 3.2 Suppose $\left(P, \leq_{P}\right.$, chain $: P \longrightarrow\{1, \ldots, m\}$, color $\left.: P \longrightarrow\{\alpha, \beta\}\right)$ is a two-color grid poset with the max property. Let $\mathbf{t} \in L=J_{\text {color }}(P)$. For $\gamma \in\{\alpha$, $\beta$, let $\mathbf{t}^{\left(i_{1}\right)}, \ldots, \mathbf{t}^{\left(i_{k}\right)}$ with $1 \leq i_{1}<\cdots<i_{k} \leq m$ be all of the descendants of $\mathbf{t}$ in $L$ for which $\mathbf{t}^{\left(i_{p}\right)} \stackrel{\gamma}{\rightarrow} \mathbf{t}$, where $1 \leq p \leq k$. Then ancestor $_{L}\left(\mathbf{t}^{\left(i_{p}\right)}\right) \neq \mathbf{t}$ for $1 \leq p<k$.

In the language of [DLP2], when a two-color grid poset $P$ has the max property, Lemma 3.2 implies that $L=J_{\text {color }}(P)$ together with the total ordering $\mathcal{T}_{L}$ and ancestor function ancestor $_{L}$ will have no "exceptional descendants" and, in light of part (4) of Lemma 3.1, will be "diamond-and-crossing friendly." These are the crucial facts needed in order to apply Theorem 4.1 of [DLP2] in the proof of Theorem 4.4.

Proof of Lemma 3.2. Without loss of generality we may assume that chain is surjective. Suppose $1 \leq p<k$. Let $\mathbf{s}:=\mathbf{t}^{\left(i_{p}\right)}$. For $i_{p}+1 \leq j \leq m, \mathcal{C}_{j} \backslash \mathbf{s}=\mathcal{C}_{j} \backslash \mathbf{t}$. We claim that for some $j$ with $i_{p}+1 \leq j \leq m$, it is the case that $\mathcal{C}_{j} \backslash \mathbf{s} \neq \emptyset$. Otherwise, suppose that $\mathcal{C}_{j} \backslash \mathbf{s}=\emptyset$ for all $i_{p}+1 \leq j \leq m$. We let $u$ be the unique vertex in chain $\mathcal{C}_{i_{k}}$ for which $\{u\}=\mathbf{t} \backslash \mathbf{t}^{\left(i_{k}\right)}$. If $u \rightarrow u^{\prime}$ for some $u^{\prime}$ in $\mathcal{C}_{i_{k}-1}$, then since $i_{p}+1 \leq i_{k}-1$ and therefore $\mathbf{t} \cap \mathcal{C}_{i_{k}-1}=\mathcal{C}_{i_{k}-1}$, it follows that $u^{\prime} \in \mathbf{t}$. But then $\mathbf{t}^{\left(i_{k}\right)}=\mathbf{t} \backslash\{u\}$ will not be an order ideal. Use similar reasoning to see that there is no $u^{\prime \prime}$ in $\mathcal{C}_{i_{k}}$ for which $u \rightarrow u^{\prime \prime}$. Therefore $u$ is maximal in $P$. Now $1 \leq i_{p}<i_{k}$. If $i_{k}>2$, then we have a maximal vertex in $P$ which is not in $\mathcal{C}_{1} \cup \mathcal{C}_{2}$, contradicting the fact that $P$ has the max property. If $i_{k}=2$, then since the chains $\mathcal{C}_{i_{p}}$ and $\mathcal{C}_{i_{k}}$ have the same color, it must be the case that chains $\mathcal{C}_{1}$ and $\mathcal{C}_{2}$ are in different connected components of $P$. In particular, $\mathcal{C}_{1}$ will be in a connected component of its own. Then there will be at least two maximal vertices of color $\gamma$. But again this contradicts the fact that $P$ has the max property. So now let $j$ be the largest integer for which $i_{p}+1 \leq j \leq m$ and $\mathcal{C}_{j} \backslash \mathbf{s}=\mathcal{C}_{j} \backslash \mathbf{t} \neq \emptyset$. Let $v$ be the largest element in $\mathcal{T}_{P}$ for which $v \in \mathcal{C}_{j} \backslash \mathbf{s}$. In particular, $v$ is the largest element in $\mathcal{T}_{P}$ that is not in $\mathbf{s}$. If $w \rightarrow v$ for some $w \in P$, then either $w \in \mathbf{s} \cap \mathcal{C}_{j}$ or $w \in \mathcal{C}_{j+1}$. In either case, $w \in \mathbf{s}$. Therefore $\mathbf{s} \cup\{v\}$ is an order ideal taken from $P$, and so $\operatorname{ancestor~}_{L}(\mathbf{s})=\mathbf{s} \cup\{v\} \neq \mathbf{t}$.

The converse of Lemma 3.2 formulated in Lemma 3.3 says that whenever $P$ is a two-color grid poset without the max property, then $L=J_{\text {color }}(P)$ will have exceptional descendants. Since Lemma 3.3 is not needed elsewhere in this paper, we state the result without proof.

Lemma 3.3 Let $\left(P, \leq_{P}\right.$, chain $: P \longrightarrow\{1, \ldots, m\}$, color $\left.: P \longrightarrow\{\alpha, \beta\}\right)$ be a two-color 
grid poset without the max property. Then there exists a color $\gamma \in\{\alpha, \beta\}$ and an element $\mathbf{t}$ of $L=J_{\text {color }}(P)$ with two descendants $\mathbf{r}$ and $\mathbf{s}$ such that $\mathbf{r} \stackrel{\gamma}{\rightarrow} \mathbf{t}, \mathbf{s} \stackrel{\gamma}{\rightarrow} \mathbf{t}, \mathbf{r}$ precedes $\mathbf{s}$ in the total order $\mathcal{T}_{L}$, and ancestor $_{L}(\mathbf{r})=\mathbf{t}$.

For more on the following discussion of "decomposing" grid posets and two-color grid posets, see [ADLMPW]. Let $P$ be a grid poset with chain function chain $: P \longrightarrow$ $\{1,2, \ldots, m\}$. Suppose $P_{1}$ is a nonempty order ideal and is a proper subset of $P$. Regard $P_{1}$ and $P_{2}:=P \backslash P_{1}$ to be subposets of the poset $P$ in the induced order. Suppose that whenever $u$ is a maximal (respectively minimal) element of $P_{1}$ and $v$ is a maximal (respectively minimal) element of $P_{2}$, then $\operatorname{chain}(u) \leq \operatorname{chain}(v)$. Then we say that $P$ decomposes into $P_{1} \triangleleft P_{2}$, and we write $P=P_{1} \triangleleft P_{2}$. If no such order ideal $P_{1}$ exists, then we say the grid poset $P$ is indecomposable. If $P$ is a grid poset that decomposes into $P_{1} \triangleleft Q$, and if $Q$ decomposes into $P_{2} \triangleleft P_{3}$, then $P=P_{1} \triangleleft\left(P_{2} \triangleleft P_{3}\right)$. But now observe that $P=\left(P_{1} \triangleleft P_{2}\right) \triangleleft P_{3}$. So we may write $P=P_{1} \triangleleft P_{2} \triangleleft P_{3}$ unambiguously. In general, if $P=P_{1} \triangleleft P_{2} \triangleleft \cdots \triangleleft P_{k}$, then each $P_{i}$ with chain function chain $\left.\right|_{P_{i}}$ is a grid subposet of $P$. If in addition $P$ is a two-color grid poset with two-color function color, then each $P_{i}$ with chain function chain $\left.\right|_{P_{i}}$ and two-color function color $\left.\right|_{P_{i}}$ is a two-color grid subposet of $P$, and so $P_{1} \triangleleft P_{2} \triangleleft \cdots \triangleleft P_{k}$ is a decomposition of $P$ into two-color grid posets.

For the remainder of this paper, we let $\mathfrak{g}$ denote a rank two semisimple Lie algebra, we identify $\alpha$ with a short simple root for $\mathfrak{g}$, and we identify $\beta$ as the other simple root. The vertex colors and edge colors for the posets and lattices we now present are simple roots. Let $\omega_{\alpha}=\omega_{1}=(1,0)$ and $\omega_{\beta}=\omega_{2}=(0,1)$ respectively denote the corresponding fundamental weights. Any weight $\mu$ in $\Lambda$ of the form $\mu=p \omega_{\alpha}+q \omega_{\beta}$ (where $p$ and $q$ are integers) is now identified with the pair $(p, q)$ in $\mathbb{Z} \times \mathbb{Z}$. Then $\alpha$ and $\beta$ are respectively identified with the first and second row vectors from the Cartan matrix for $\mathfrak{g}$ :

Figure 3.1

\begin{tabular}{|c|c||c|c|}
\hline$A_{1} \times A_{1}$ & $\left(\begin{array}{ll}2 & 0 \\
0 & 2\end{array}\right)$ & $A_{2}$ & $\left(\begin{array}{cc}2 & -1 \\
-1 & 2\end{array}\right)$ \\
\hline$C_{2}$ & $\left(\begin{array}{cc}2 & -1 \\
-2 & 2\end{array}\right)$ & $G_{2}$ & $\left(\begin{array}{cc}2 & -1 \\
-3 & 2\end{array}\right)$ \\
\hline
\end{tabular}

In this notation, we define the $\mathfrak{g}$-fundamental posets $P_{\mathfrak{g}}(1,0)$ and $P_{\mathfrak{g}}(0,1)$ to be the twocolor grid posets of Figure 3.2. The corresponding $\mathfrak{g}$-fundamental lattices are the edgecolored lattices $L_{\mathfrak{g}}(1,0):=J_{\text {color }}\left(P_{\mathfrak{g}}(1,0)\right)$ and $L_{\mathfrak{g}}(0,1):=J_{\text {color }}\left(P_{\mathfrak{g}}(0,1)\right)$ respectively. Now let $\lambda=(a, b)$ be a pair of nonnegative integers. There are exactly two possible ways that a two-color grid poset $P$ with the max property can decompose as $P_{1} \triangleleft P_{2} \triangleleft \cdots \triangleleft P_{a+b}$ with $a$ of the $P_{i}$ 's vertex-color isomorphic to $P_{\mathfrak{g}}(1,0)$ and the remaining $P_{i}$ 's vertex-color isomorphic to $P_{\mathfrak{g}}(0,1)$ : we will either have $P_{i}$ isomorphic to $P_{\mathfrak{g}}(0,1)$ for $1 \leq i \leq b$ and isomorphic to $P_{\mathfrak{g}}(1,0)$ for $1+b \leq i \leq a+b$ (in which case we set $P_{\mathfrak{g}}^{\beta \alpha}(\lambda):=P$ ), or we will have $P_{i}$ isomorphic to $P_{\mathfrak{g}}(1,0)$ for $1 \leq i \leq a$ and isomorphic to $P_{\mathfrak{g}}(0,1)$ for $a+1 \leq i \leq a+b$ (in which case we set $P_{\mathfrak{g}}^{\alpha \beta}(\lambda):=P$ ). Note that $P_{\mathfrak{g}}^{\beta \alpha}(1,0)=P_{\mathfrak{g}}^{\alpha \beta}(1,0)=P_{\mathfrak{g}}(1,0)$, and $P_{\mathfrak{g}}^{\beta \alpha}(0,1)=P_{\mathfrak{g}}^{\alpha \beta}(0,1)=P_{\mathfrak{g}}(0,1)$. When $a=b=0$, then $P_{\mathfrak{g}}^{\beta \alpha}(\lambda)$ and $P_{\mathfrak{g}}^{\alpha \beta}(\lambda)$ are the empty set. We call $P_{\mathfrak{g}}^{\beta \alpha}(\lambda)$ and $P_{\mathfrak{g}}^{\alpha \beta}(\lambda)$ the $\mathfrak{g}$-semistandard posets associated to $\lambda$. For 
each semisimple Lie algebra $\mathfrak{g}, P_{\mathfrak{g}}^{\beta \alpha}(2,2)$ is depicted in Figure $3.3 ; P_{\mathfrak{g}}^{\alpha \beta}(2,2)$ is depicted in Figure 3.4. The $\mathfrak{g}$-semistandard lattices associated to $\lambda$ are the edge-colored lattices $L_{\mathfrak{g}}^{\beta \alpha}(\lambda):=J_{\text {color }}\left(P_{\mathfrak{g}}^{\beta \alpha}(\lambda)\right)$ and $L_{\mathfrak{g}}^{\alpha \beta}(\lambda):=J_{\text {color }}\left(P_{\mathfrak{g}}^{\alpha \beta}(\lambda)\right)$. Note that $L_{\mathfrak{g}}^{\beta \alpha}(1,0)=L_{\mathfrak{g}}^{\alpha \beta}(1,0)=$ $L_{\mathfrak{g}}(1,0)$, and $L_{\mathfrak{g}}^{\beta \alpha}(0,1)=L_{\mathfrak{g}}^{\alpha \beta}(0,1)=L_{\mathfrak{g}}(0,1)$.

Figure 3.2: Fundamental posets.

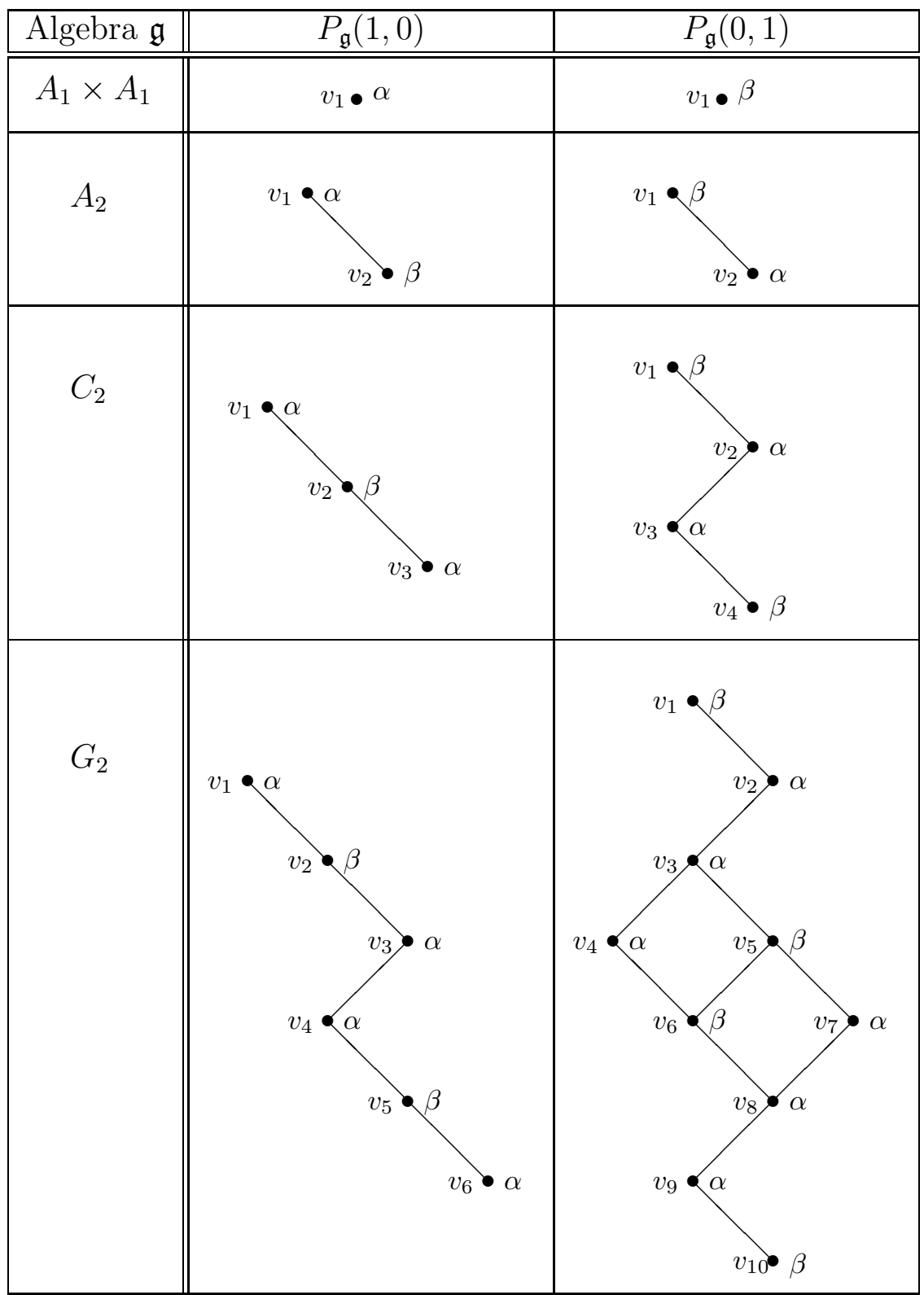


Figure 3.3: Depicted below are four two-color grid posets each possessing the max property. (Each is the $\mathfrak{g}$-semistandard poset $P_{\mathfrak{g}}^{\beta \alpha}(2,2)$ for the indicated rank two semisimple Lie algebra $\mathfrak{g}$.)

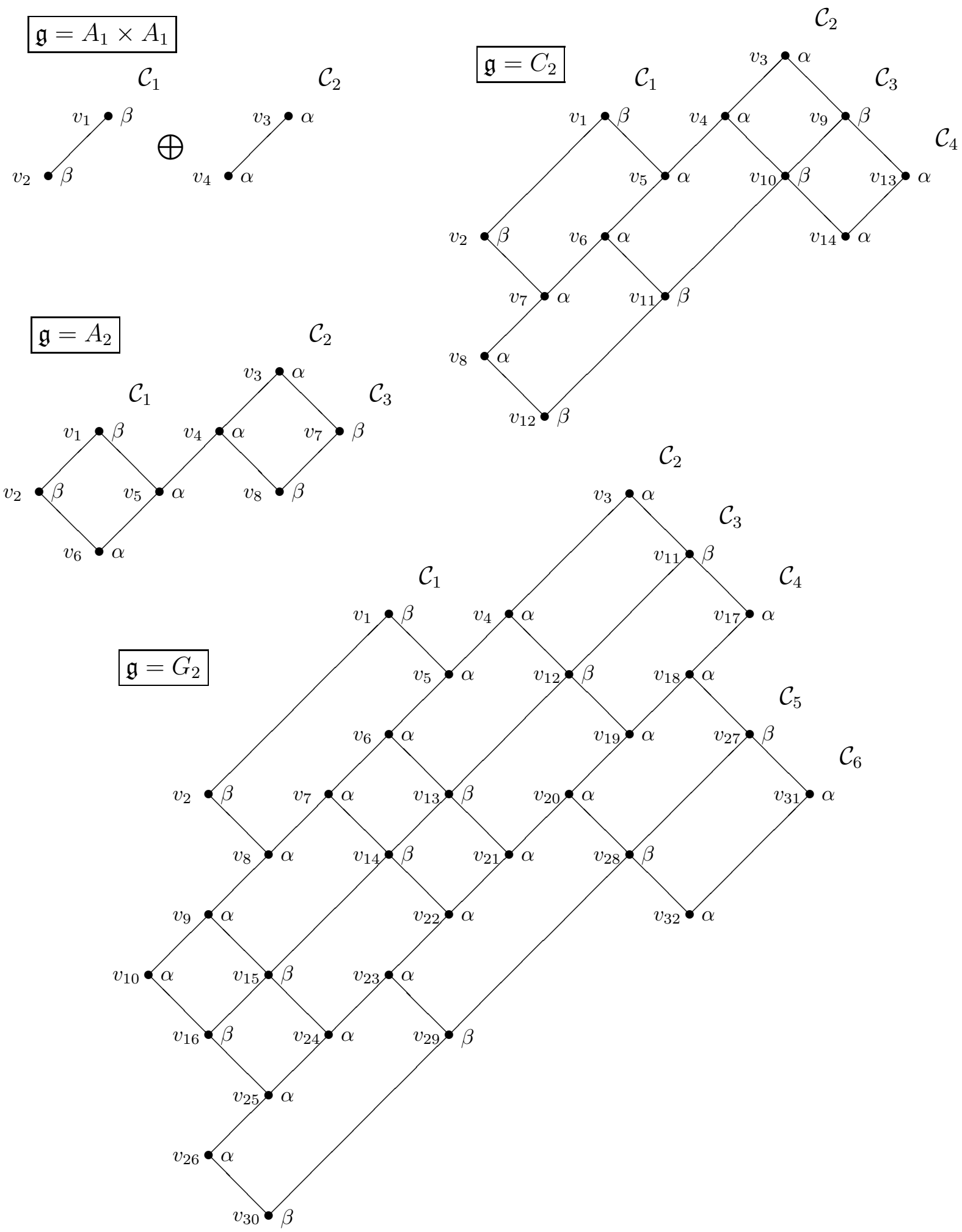


Figure 3.4: Depicted below are four two-color grid posets each possessing the max property. (Each is the $\mathfrak{g}$-semistandard poset $P_{\mathfrak{g}}^{\alpha \beta}(2,2)$ for the indicated rank two semisimple Lie algebra $\mathfrak{g}$.)

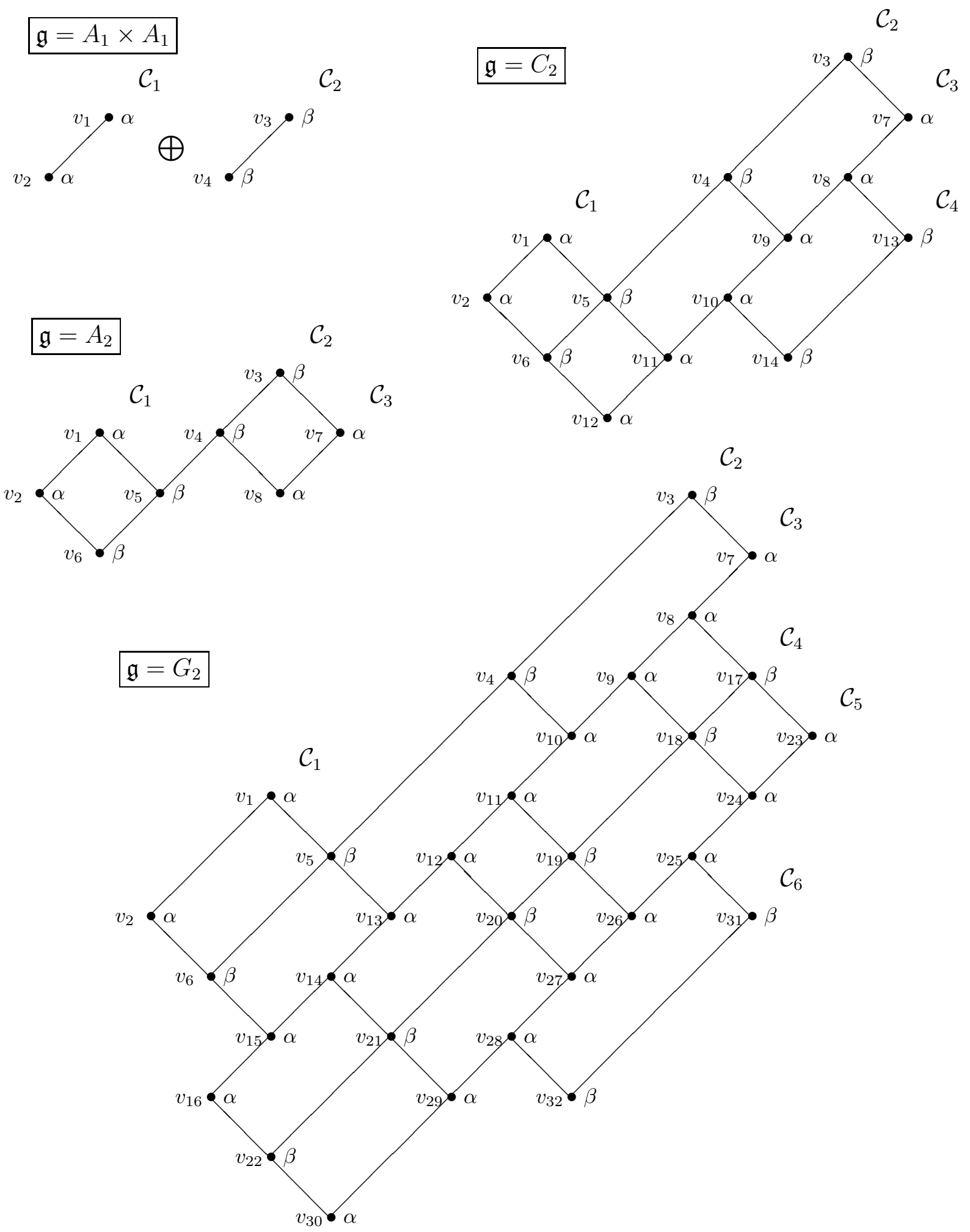




\section{Semistandard lattices as supporting graphs}

Although their significance to us is primarily Lie theoretic, the results of this section are consequences of the combinatorics of semistandard posets and semistandard lattices. The main result of this section (Theorem 4.4) uses general principles to show that semistandard lattices enjoy the edge-minimal (extremal) and solitary (uniqueness) properties for supporting graphs conditional on the existence of certain edge coefficients; Theorem 5.1 addresses the existence question. From here on, when $L$ is either of the $\mathfrak{g}$ semistandard lattices $L_{\mathfrak{g}}^{\beta \alpha}(\lambda)$ or $L_{\mathfrak{g}}^{\alpha \beta}(\lambda)$, then for each $\mathbf{s}$ in $L$ we will refer to the quantity $w t_{L}(\mathbf{s})=\left(m_{\alpha}(\mathbf{s}), m_{\beta}(\mathbf{s})\right)$ as the "weight of $\mathbf{s} "$ and use the notation $w t(\mathbf{s})$. The following result is Proposition 4.2 of [ADLMPW].

Proposition 4.1 Let $\lambda=(a, b)$ be a pair of nonnegative integers, and let $L$ be one of the $\mathfrak{g}$-semistandard lattices $L_{\mathfrak{g}}^{\beta \alpha}(\lambda)$ or $L_{\mathfrak{g}}^{\alpha \beta}(\lambda)$. Let $\mathbf{s} \stackrel{\gamma}{\rightarrow} \mathbf{t}$ be an edge of color $\gamma \in\{\alpha, \beta\}$ in $L$. Then $w t(\mathbf{s})+\gamma=w t(\mathbf{t})$, and hence $L$ satisfies the $\mathfrak{g}$-structure condition.

Proposition 4.2 Let $\lambda=(a, b)$ be any pair of nonnegative integers. If $\mathfrak{g}=A_{1} \times A_{1}$, then $\left|L_{\mathfrak{g}}^{\beta \alpha}(\lambda)\right|=\left|L_{\mathfrak{g}}^{\alpha \beta}(\lambda)\right|=\frac{1}{1 !}(a+1)(b+1)$. If $\mathfrak{g}=A_{2}$, then $\left|L_{\mathfrak{g}}^{\beta \alpha}(\lambda)\right|=\left|L_{\mathfrak{g}}^{\alpha \beta}(\lambda)\right|=\frac{1}{2 !}(a+1)(b+$ 1) $(a+b+2)$. If $\mathfrak{g}=C_{2}$, then $\left|L_{\mathfrak{g}}^{\beta \alpha}(\lambda)\right|=\left|L_{\mathfrak{g}}^{\alpha \beta}(\lambda)\right|=\frac{1}{3 !}(a+1)(b+1)(a+b+2)(a+2 b+3)$. If $\mathfrak{g}=G_{2}$, then $\left|L_{\mathfrak{g}}^{\beta \alpha}(\lambda)\right|=\left|L_{\mathfrak{g}}^{\alpha \beta}(\lambda)\right|=\frac{1}{5 !}(a+1)(b+1)(a+b+2)(a+2 b+3)(a+3 b+$ 4) $(2 a+3 b+5)$.

Proof. Apply Proposition 4.5 of [ADLMPW] (restated below as Proposition 5.2). Each formula can be worked out by hand using standard enumerative techniques to count the $\mathfrak{g}$-semistandard tableaux corresponding to the elements of $L_{\mathfrak{g}}^{\beta \alpha}(\lambda)$. In the $G_{2}$ case we used a computer algebra system to simplify some of the identities involved. In the $A_{2}$ and $C_{2}$ cases these formulas were verified in $[\mathrm{Alv}]$. Since in all cases $L_{\mathfrak{g}}^{\alpha \beta}(\lambda)$ is poset-isomorphic to the dual poset $\left(L_{\mathfrak{g}}^{\beta \alpha}(\lambda)\right)^{*}$, it follows that $\left|L_{\mathfrak{g}}^{\alpha \beta}(\lambda)\right|=\left|L_{\mathfrak{g}}^{\beta \alpha}(\lambda)\right|$.

We note that this proposition also follows from Theorem 5.3 of [ADLMPW] together with the Weyl degree formula (see also Corollary 5.4 of that paper); however, in applying Proposition 4.2 in the proof of Proposition 4.3, we want a proof that is independent of Theorem 5.3 of [ADLMPW].

Proposition 4.3 Let $\lambda=(a, b)$ for nonnegative integers $a$ and $b$, at least one of which is positive. Let $L$ be one of the $\mathfrak{g}$-semistandard lattices $L_{\mathfrak{g}}^{\beta \alpha}(\lambda)$ or $L_{\mathfrak{g}}^{\alpha \beta}(\lambda)$. Suppose $L$ is a supporting graph for a representation of some rank two semisimple Lie algebra $\mathfrak{g}^{\prime}$. Then $\mathfrak{g}^{\prime} \cong \mathfrak{g}$ and $L$ is a supporting graph for an irreducible representation of $\mathfrak{g}$ with highest weight $\lambda$. When $\lambda=(0,0)$, each lattice $L_{\mathfrak{g}}^{\beta \alpha}(\lambda)$ and $L_{\mathfrak{g}}^{\alpha \beta}(\lambda)$ has one element and is a supporting graph for an irreducible representation of $\mathfrak{g}$ with highest weight $\lambda$.

Proof. When $\lambda=(0,0)$, each one-element lattice $L_{\mathfrak{g}}^{\beta \alpha}(\lambda)$ and $L_{\mathfrak{g}}^{\alpha \beta}(\lambda)$ vacuously satisfies the requirements of Proposition 2.2 and hence is a supporting graph for a $\mathfrak{g}$-module; a one-dimensional $\mathfrak{g}$-module is necessarily irreducible with highest weight $\lambda=(0,0)$.

Now let $\lambda=(a, b)$ for nonnegative integers $a$ and $b$, at least one of which is positive, and let $L$ be one of the $\mathfrak{g}$-semistandard lattices $L_{\mathfrak{g}}^{\beta \alpha}(\lambda)$ or $L_{\mathfrak{g}}^{\alpha \beta}(\lambda)$. By Proposition $4.1, L$ satisfies the $\mathfrak{g}$-structure condition. By hypothesis, $L$ satisfies the $\mathfrak{g}^{\prime}$-structure condition. If $\mathfrak{g}$ is simple, then one can see by inspecting the corresponding semistandard poset $P$ that, as long as one of $a$ or $b$ is positive, $L$ has at least one edge of color $\alpha$ and at least one edge 
of color $\beta$; the same is true if $\mathfrak{g}=A_{1} \times A_{1}$ and $a$ and $b$ are both positive. In these cases it follows from Lemma 2.1 that $\mathfrak{g}^{\prime} \cong \mathfrak{g}$. If $\mathfrak{g}=A_{1} \times A_{1}$ and $a=0$ or $b=0$, then all edges of $L$ have the same color, and hence $L$ is a supporting graph for a representation of $A_{1}$. The only way $L$ can also be a supporting graph for a semisimple Lie algebra $\mathfrak{g}^{\prime}$ of rank two is if $\mathfrak{g}^{\prime} \cong A_{1} \times A_{1}$. Now we identify the representation $V$ of $\mathfrak{g}$ which has $L$ as its supporting graph. First, note that the maximal element $\mathbf{m}$ for $L$ corresponds to a maximal vector in $V$ with weight $w t(\mathbf{m})=(a, b)=\lambda$. Thus one of the components in the decomposition of $V$ as a direct sum of irreducible $\mathfrak{g}$-modules is of highest weight $\lambda$. However, Proposition 4.2 and the Weyl degree formula show that the quantity $|L|=\operatorname{dim} V$ is the same as the dimension of any irreducible $\mathfrak{g}$-module with highest weight $\lambda$. Then $V$ must be irreducible with highest weight $\lambda$.

Theorem 4.4 Let $\lambda=(a, b)$ be a pair of nonnegative integers. Let $L$ be one of the $\mathfrak{g}$-semistandard lattices $L_{\mathfrak{g}}^{\beta \alpha}(\lambda)$ or $L_{\mathfrak{g}}^{\beta \alpha}(\lambda)$. Suppose there exists a set of nonzero coefficients $\left\{\left(X_{\mathbf{t}, \mathbf{s}}, Y_{\mathbf{s}, \mathbf{t}}\right)\right\}_{\mathbf{s} \rightarrow \mathbf{i} \in \mathcal{E}(L)}$ that can be assigned to the edges of $L$ in such a way that the corresponding edge-labelled poset satisfies the diamond and crossing conditions.

(1) Suppose $\left\{\left(c_{\mathbf{t}, \mathbf{s}}, d_{\mathbf{s}, \mathbf{t}}\right)\right\}_{\mathbf{s} \rightarrow \mathbf{i} \in \mathcal{E}(L)}$ is another set of coefficients that can be assigned to the edges of $L$ such that the corresponding edge-labelled poset satisfies the diamond and crossing conditions. Then on any edge $\mathbf{s} \stackrel{i}{\rightarrow} \mathbf{t}$ in $L$, it is the case that $c_{\mathbf{t}, \mathbf{s}} d_{\mathbf{s}, \mathbf{t}}=X_{\mathbf{t}, \mathbf{s}} Y_{\mathbf{s}, \mathbf{t}}$.

(2) The edge-colored poset $L$ is a solitary and edge-minimal supporting graph for an irreducible representation of $\mathfrak{g}$ with highest weight $\lambda$.

Proof. Let $P$ be one of $P_{\mathfrak{g}}^{\beta \alpha}(\lambda)$ or $P_{\mathfrak{g}}^{\alpha \beta}(\lambda)$, with $L$ the corresponding semistandard lattice. Apply Lemma 3.2 to the two-color grid poset $P$ to see that, in the language of [DLP2], $L$ together with $\mathcal{T}_{L}$ and ancestor $_{L}$ has no exceptional descendants. Then using part (4) of Lemma 3.1 it now follows that $L$ together with $\mathcal{T}_{L}$ and ancestor $_{L}$ is diamondand-crossing friendly. Now apply part (1) of Theorem 4.1 of [DLP2] to get part (1) of this theorem. In light of Proposition 4.3, we may apply part (2) of Theorem 4.1 of [DLP2] to get part (2) of this theorem.

Corollary 4.5 Let $\lambda=(a, b)$ be a pair of nonnegative integers, and let $\mathfrak{g}$ be simple. Suppose $L_{\mathfrak{g}}^{\beta \alpha}(\lambda)$ and $L_{\mathfrak{g}}^{\alpha \beta}(\lambda)$ both meet the hypotheses of Theorem 4.4. Suppose two weight bases for an irreducible representation of $\mathfrak{g}$ with highest weight $\lambda$ have supporting graphs $L_{\mathfrak{g}}^{\beta \alpha}(\lambda)$ and $L_{\mathfrak{g}}^{\alpha \beta}(\lambda)$ respectively. Then the bases are diagonally equivalent if and only if $a=0$ or $b=0$.

Proof. Under these assumptions, it follows from Theorem 4.4 that the semistandard lattices $L_{\mathfrak{g}}^{\beta \alpha}(\lambda)$ and $L_{\mathfrak{g}}^{\alpha \beta}(\lambda)$ are solitary. Suppose $a=0$ or $b=0$. Then by Lemma 4.3 , we have $L_{\mathfrak{g}}^{\beta \alpha}(\lambda) \cong L_{\mathfrak{g}}^{\alpha \beta}(\lambda)$, so the corresponding weight bases are diagonally equivalent. Conversely, suppose two weight bases with respective supporting graphs $L_{\mathfrak{g}}^{\beta \alpha}(\lambda)$ and $L_{\mathfrak{g}}^{\alpha \beta}(\lambda)$ are diagonally equivalent. Then $L_{\mathfrak{g}}^{\beta \alpha}(\lambda)$ and $L_{\mathfrak{g}}^{\alpha \beta}(\lambda)$ are isomorphic as edge-colored posets by Lemma 3.1.B of [Don1]. So by Lemma 4.3 of [ADLMPW] we have $a=0$ or $b=0$.

Lemma 4.6 Let $\lambda=(a, b)$ for nonnegative integers $a$ and $b$. Then $L_{\mathfrak{g}}^{\beta \alpha}(\lambda)$ is a supporting graph for $\mathfrak{g}$ if and only if $L_{\mathfrak{g}}^{\alpha \beta}(\lambda)$ is a supporting graph for $\mathfrak{g}$. In this case, $L_{\mathfrak{g}}^{\beta \alpha}(\lambda)$ is positive rational (respectively positive integral, solitary, edge-minimal, or edge-minimizing) if and only if $L_{\mathfrak{g}}^{\alpha \beta}(\lambda)$ is positive rational (respectively positive integral, solitary, edge-minimal, or edge-minimizing). 
Proof. It follows from Proposition 2.5 that $L_{\mathfrak{g}}^{\beta \alpha}(\lambda)$ is a supporting graph for $\mathfrak{g}$ if and only if $\left(L_{\mathfrak{g}}^{\beta \alpha}(\lambda)\right)^{\triangle}$ is a supporting graph for $\mathfrak{g}$. Observations from the paragraph preceding Lemma 4.3 of [ADLMPW] show that $L_{\mathfrak{g}}^{\alpha \beta}(\lambda) \cong\left(L_{\mathfrak{g}}^{\beta \alpha}(\lambda)\right)^{\triangle}$ as edge-colored posets. This proves the first assertion of the lemma. We may now apply Proposition 2.5 to see that $L_{\mathfrak{g}}^{\beta \alpha}(\lambda)$ is positive rational (respectively positive integral, solitary, edge-minimal, or edge-minimizing) if and only if $L_{\mathfrak{g}}^{\alpha \beta}(\lambda)$ is positive rational (respectively positive integral, solitary, edge-minimal, or edge-minimizing).

For a rank two semisimple Lie algebra $\mathfrak{g}$, let $V$ be an irreducible $\mathfrak{g}$-module with highest weight $\lambda=a \omega_{\alpha}+b \omega_{\beta}=(a, b)$. Let $L$ be one of $L_{\mathfrak{g}}^{\beta \alpha}(\lambda)$ or $L_{\mathfrak{g}}^{\alpha \beta}(\lambda)$, and let $\rho$ be its rank

function. Then by Theorem 5.3 of $[\mathrm{ADLMPW}]$, for any weight $\mu \in \Lambda, \operatorname{dim}\left(V_{\mu}\right)=\mid\{\mathbf{s} \in$ $L \mid w t(\mathbf{s})=\mu\} \mid$. Form an edge-colored directed graph $M_{\mathfrak{g}}(\lambda)$ whose vertices are the elements of $L$ and whose edges of color $\gamma(\gamma \in\{\alpha, \beta\})$ are determined by the rule $\mathbf{s} \stackrel{\gamma}{\rightarrow} \mathbf{t}$ if and only if $w t(\mathbf{s})+\gamma=w t(\mathbf{t})$. It now follows from the discussion of Section 3.1 of [Don1] that $M_{\mathfrak{g}}(\lambda)$ is isomorphic as an edge-colored directed graph to the unique maximal supporting graph for $V$. (In [Don1] it is observed that almost all weight bases for $V$ have the unique maximal supporting graph as their supporting graph.) In particular, the isomorphism class of $M_{\mathfrak{g}}(\lambda)$ does not depend on the choice of $L_{\mathfrak{g}}^{\beta \alpha}(\lambda)$ or $L_{\mathfrak{g}}^{\alpha \beta}(\lambda)$ as a starting point. Thus we have:

Proposition 4.7 Let $\lambda=(a, b)$ be a pair of nonnegative integers. Let $\mathfrak{g}$ be a semisimple Lie algebra of rank two. Let $L$ be one of the $\mathfrak{g}$-semistandard lattices $L_{\mathfrak{g}}^{\beta \alpha}(\lambda)$ and $L_{\mathfrak{g}}^{\alpha \beta}(\lambda)$. Then $M_{\mathfrak{g}}(\lambda)$ obtained from $L$ as in the preceding paragraph is isomorphic as an edgecolored directed graph to the unique maximal supporting graph for any irreducible $\mathfrak{g}$ module of highest weight $\lambda$.

\section{Classification of semistandard lattice supporting graphs}

The main result of this section (Theorem 5.1) is a classification/existence result: We classify those semistandard lattices which are supporting graphs, and in each such case we obtain (or say where one can obtain) edge coefficients which explicitly describe Chevalley generator actions on a weight basis.

Theorem 5.1 Let $a$ and $b$ be nonnegative integers. For $\mathfrak{g}=A_{1} \times A_{1}$ or $\mathfrak{g}=A_{2}$ and for $\lambda=(a, b)$, each $\mathfrak{g}$-semistandard lattice $L_{\mathfrak{g}}^{\beta \alpha}(\lambda)$ and $L_{\mathfrak{g}}^{\alpha \beta}(\lambda)$ is a supporting graph for an irreducible representation of $\mathfrak{g}$ with highest weight $\lambda$. Each $C_{2}$-semistandard lattice $L_{C_{2}}^{\beta \alpha}(\lambda)$ and $L_{C_{2}}^{\alpha \beta}(\lambda)$ is a supporting graph for an irreducible representation of $C_{2}$ with highest weight $\lambda$ if $\lambda=(a, 0), \lambda=(0, b)$, or $\lambda=(1, b)$; otherwise $L_{C_{2}}^{\beta \alpha}(\lambda)$ and $L_{C_{2}}^{\alpha \beta}(\lambda)$ are not supporting graphs for $C_{2}$. Each $G_{2}$-semistandard lattice $L_{G_{2}}^{\beta \alpha}(\lambda)$ and $L_{G_{2}}^{\alpha \beta}(\lambda)$ is a supporting graph for an irreducible representation of $G_{2}$ with highest weight $\lambda$ if $\lambda=(a, 0)$ or $\lambda=(0,1)$; otherwise $L_{G_{2}}^{\beta \alpha}(\lambda)$ and $L_{G_{2}}^{\alpha \beta}(\lambda)$ are not supporting graphs for $G_{2}$. If a $\mathfrak{g}$ semistandard lattice is a supporting graph for $\mathfrak{g}$, then it is positive rational, solitary, and edge-minimal.

The case-by-case proof is at the end of this section and requires some preliminary 
results. Three parts of this theorem are new and are proved here. First, our constructions in Proposition 5.6 of two families of weight bases (one each for $L_{C_{2}}^{\beta \alpha}(\lambda)$ and $L_{C_{2}}^{\alpha \beta}(\lambda)$ ) in the $C_{2}$ case for irreducible representations with highest weight $\lambda=(1, b)$ with $b \geq 1$ appear to be new. Second, the combinatorial construction of a weight basis for the $C_{2}$ irreducible representation with highest weight $\lambda=(a, 0)(a \geq 0)$ gives a new perspective on a wellknown basis. And third, we show in Proposition 5.7 (following [Alv]) and Proposition 5.8 why the semistandard lattices listed in Theorem 5.1 are the only semistandard lattices that can serve as supporting graphs. The remaining parts of this theorem have appeared in previous papers, but the semistandard viewpoint at hand now "explains" the families of weight bases covered by Theorem 5.1 from just one perspective.

In Proposition 5.6 we use our $C_{2}$-semistandard lattices to explicitly construct two new positive rational weight bases for each irreducible representation of $C_{2}$ with highest weight $\lambda=(1, b)$ for $b \geq 1$. Our bases for these representations are different from Molev's $C_{2}$ weight bases from [Mol1] and his $B_{2}$ weight bases from [Mol2] for the following reasons. Implicit in Molev's weight basis constructions for irreducible representations of $C_{n} \approx \mathfrak{s p}(2 n, \mathbb{C})$ (respectively $\left.B_{n} \approx \mathfrak{o}(2 n+1, \mathbb{C})\right)$ is a choice of Chevalley generators; a key property these weight bases possess is that (in the language of [Don1]) they restrict irreducibly for the chain $C_{n} \supset C_{n-1} \supset \cdots \supset C_{2} \supset C_{1}=A_{1}$ (respectively $B_{n} \supset B_{n-1} \supset$ $\cdots \supset B_{2} \supset B_{1}=A_{1}$ ). In certain cases (for example a weight basis for the fundamental representations of $\mathfrak{s p}(2 n, \mathbb{C})$ studied in [Don1]) it can be seen that the weight basis is uniquely determined by this property. In rank two, this restriction property implies that in the supporting graph for a Molev basis, for some color all of the components should be chains. This is not the case for any of the supporting graphs in the $L_{C_{2}}^{\beta \alpha}(1, b)$ and $L_{C_{2}}^{\alpha \beta}(1, b)$ families of lattices when $b \geq 1$; for $\lambda=(1, b)=(1,1)$ this is readily observed from the picture in Figure 6.1. Thus, these $C_{2}$-semistandard lattices are not supporting graphs for $B_{2}$ or $C_{2}$ Molev bases. We note that since our bases do not possess this restriction property, the methods of [Don1] cannot be used to show these bases are solitary and edge-minimal.

In Proposition 5.5 we use the $C_{2}$-semistandard lattices to construct positive integral weight bases for irreducible representations of $C_{2}$ corresponding to highest weights of the form $(a, 0)$. While the combinatorial perspective of this proposition is new, these representations are easily constructed when viewed as symmetric powers of the defining four-dimensional irreducible representation of $C_{2}$. Although we will not do so here, it can be shown that the bases obtained here are diagonally equivalent to the respective bases for these representations constructed in [Mol1]. This result easily generalizes from $C_{2}$ to $C_{n} \cdot^{\dagger}$

\footnotetext{
†Briefly, for an integer $a \geq 1$ take the "factorial normalized" monomial basis for the ath symmetric power $V^{a}$ of the defining $2 n$-dimensional representation $V$ of $\mathfrak{s l}(2 n, \mathbb{C})$. Identify $\mathfrak{s l}(2 n, \mathbb{C})$ with $A_{2 n-1}$, a rank $2 n-1$ simple Lie algebra with generators $\left\{x_{i}, y_{i}\right\}_{i=1}^{2 n-1}$. The representation $V^{a}$ has highest weight $a \bar{\omega}_{1}$, where $\bar{\omega}_{1}$ is the highest weight for the defining representation $V$ of $A_{2 n-1} \approx \mathfrak{s l}(2 n, \mathbb{C})$. Let $C_{n} \approx \mathfrak{s p}(2 n, \mathbb{C})$ have generators $\left\{x_{i}^{\prime}, y_{i}^{\prime}\right\}_{i=1}^{n}$. The mapping for which $x_{i}^{\prime} \mapsto x_{i}+x_{2 n-i}$ and $y_{i}^{\prime} \mapsto y_{i}+y_{2 n-i}$ when $1 \leq i<n$ and for which $x_{n}^{\prime} \mapsto x_{n}$ and $y_{n}^{\prime} \mapsto y_{n}$ allows us to view $C_{n}$ as a Lie subalgebra of $A_{2 n-1}$. Now one can check that $V^{a}$ remains irreducible under the induced action of $C_{n}$ and has highest weight $a \omega_{1}$, where the fundamental weight $\omega_{1}$ is the highest weight for the defining representation of $C_{n} \approx \mathfrak{s p}(2 n, \mathbb{C})$. Our
} 
We will say how the results of Theorem 5.1 for $\mathfrak{g}=A_{2}$ and $\mathfrak{g}=A_{1} \times A_{1}$ can be found in Sections 4 and 6 of [Don1] respectively. We will show how the Theorem 5.1 results for $\mathfrak{g}=C_{2}$ with $\lambda=(0, b)$ and $\mathfrak{g}=G_{2}$ with $\lambda=(a, 0)$ follow from [DLP1]. (In these references one can see that these $A_{2}$ and $C_{2}$ constructions are part of families of constructions that generalize from $A_{2}$ to $A_{n}$ and from $C_{2}$ to $B_{n}$ respectively). A construction of the adjoint representation of $G_{2}$ given in [Don2] covers the case $\mathfrak{g}=G_{2}$ with $\lambda=(0,1)$; that construction is reproduced here in Proposition 5.4 together with Figure 5.2. A consequence of Part (2) of Theorem 4.4 is that any weight basis for an irreducible representation of $\mathfrak{g}$ whose supporting graph is a semistandard lattice $L$ from the list in Theorem 5.1 will be solitary and edge-minimal. Using case-by-case arguments, this was already known for all of the following semistandard lattices; in these references connections with Molev's bases from [Mol1] and [Mol2] are noted.

\begin{tabular}{|c|c|c|}
\hline Algebra & Which $\lambda=(a, b) ?$ & Reference \\
\hline$A_{1} \times A_{1}$ & $a \geq 0, b \geq 0$ & [Don1] \\
\hline$A_{2}$ & $a \geq 0, b \geq 0$ & [Don1] \\
\hline$C_{2}$ & $a=0, b \geq 0$ & {$[$ DLP1] } \\
\hline$G_{2}$ & $a \geq 0, b=0$ & {$[$ DLP2] } \\
\cline { 2 - 3 } & $a=0, b=1$ & {$[$ Don2] } \\
\hline
\end{tabular}

In working with the lattices $L_{\mathfrak{g}}^{\beta \alpha}(\lambda)$ in this section, we will freely use the identification of lattice elements with semistandard tableaux from Section 4 of [ADLMPW]. For nonnegative integers $a$ and $b$, we associate to $\lambda=(a, b)$ the following shape:

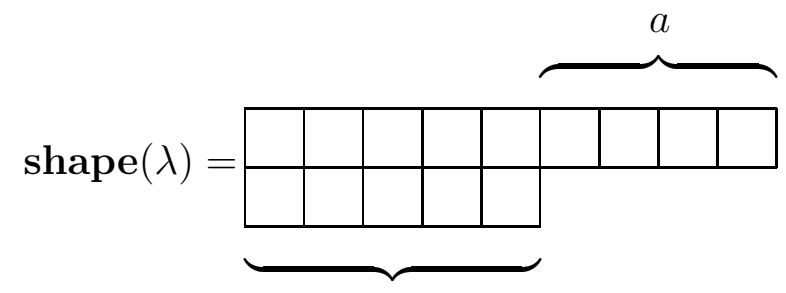

$b$

A tableau of shape $\lambda$ is a filling of all of the boxes of shape $(\lambda)$ with entries from some totally ordered set. For a tableau $T$ of shape $\lambda$, we write $T=\left(T^{(1)}, \ldots, T^{(a+b)}\right)$, where $T^{(i)}$ is the $i$ th column of $T$ counting from the left. We let $T_{j}^{(i)}$ denote the $j$ th entry of the column $T^{(i)}$, where we start counting from the top of the column. The tableau $T$ is semistandard if the entries weakly increase across rows and strictly increase down columns, i.e. $T_{j}^{(i)} \leq T_{j}^{(i+1)}$ and $T_{j}^{(i)}<T_{j+1}^{(i)}$ for all $i, j$ for which these entries of $T$ are defined. In Section 4 of [ADLMPW], to each order ideal $\mathbf{t}$ in the $\mathfrak{g}$-semistandard lattice $L_{\mathfrak{g}}^{\beta \alpha}(\lambda)$ we associated a semistandard tableau tableau $(\mathbf{t})$, there called a $\mathfrak{g}$-semistandard

chosen basis for the $A_{2 n-1}$ representation $V^{a}$ has representation diagram $L_{A}^{G T-l e f t}\left(2 n-1, a \bar{\omega}_{1}\right)$ with coefficients from Theorem 6.4 of [Don1]. It follows that to obtain the representation diagram for this basis when $V^{a}$ is viewed as a $C_{n}$-module one only needs to take $L_{A}^{G T-l e f t}\left(2 n-1, a \bar{\omega}_{1}\right)$ and recolor its edges by the rule $i \mapsto 2 n-i$ for $n+1 \leq i \leq 2 n$. When $n=2$ we get the representation $\operatorname{diagram} L_{C_{2}}^{\beta \alpha}(a, 0)$ of Proposition 5.5; a more combinatorial version of this argument is given in our proof that follows that proposition statement. 
tableau. The set $\mathcal{S}_{\mathfrak{g}}(\lambda)$ of all $\mathfrak{g}$-semistandard tableaux of shape $\lambda$ is characterized by the following result which appears as Proposition 4.5 of [ADLMPW].

Proposition 5.2 Let $a$ and $b$ be nonnegative integers, and let $\lambda=(a, b)$. Then:

$\mathcal{S}_{A_{1} \times A_{1}}(\lambda)=\{$ semistandard tableau $T$ of shape $\lambda$ with entries from $\{1,2,3\}$

$\begin{array}{|l|l}2 \\ \hline 3\end{array}$ is not a column of $T$, and 3 is not a column of $\left.T\right\}$

$\mathcal{S}_{A_{2}}(\lambda)=\{$ semistandard tableau $T$ of shape $\lambda$ with entries from $\{1,2,3\}\}$

$\mathcal{S}_{C_{2}}(\lambda)=\{$ semistandard tableau $T$ of shape $\lambda$ with entries from $\{1,2,3,4\}$

$\begin{array}{|l|l}1 \\ 4\end{array}$ is not a column of $T$, and $\frac{2}{3}$ appears at most once in $\left.T\right\}$

$\mathcal{S}_{G_{2}}(\lambda)=\{$ semistandard tableau $T$ of shape $\lambda$ with entries from $\{1,2,3,4,5,6,7\}$

the column 4 appears at most once in $T ; \frac{2}{3}, \frac{2}{4}, \frac{3}{4}, \frac{3}{5}, \frac{3}{5}, \frac{4}{6}$, and $\frac{5}{6}$

are not columns of $T$; plus the restrictions of Figure 5.1$\}$

Figure 5.1: Some restrictions for any given $G_{2}$-semistandard tableau $T$.

\begin{tabular}{|c|c|}
\hline Column $T^{(i)}$ of $T$ & Then the succeeding column $T^{(i+1)}$ of $T$ cannot be. \\
\hline 4 & 4 \\
\hline \begin{tabular}{|l|}
1 \\
4 \\
\end{tabular} & $1, \frac{1}{4}, \frac{1}{5}, \frac{1}{6}, \frac{1}{7}, \frac{1}{7}$ \\
\hline \begin{tabular}{|l|}
1 \\
5 \\
\end{tabular} & $1, \frac{1}{5}, \frac{1}{6}, \frac{1}{7}$ \\
\hline \begin{tabular}{|l|}
1 \\
6 \\
\end{tabular} & $1,2, \frac{1}{6}, \frac{1}{7}, \frac{2}{6}, \frac{2}{7}$ \\
\hline \begin{tabular}{|l|}
2 \\
6 \\
\end{tabular} & $2, \frac{2}{6}, \frac{2}{7}$ \\
\hline \begin{tabular}{|l|}
1 \\
7 \\
\end{tabular} & $1,2,3,4, \frac{1}{7}, \frac{2}{7}, \frac{3}{7}, \frac{4}{7}$ \\
\hline \begin{tabular}{|l|}
2 \\
7 \\
\end{tabular} & $2,3,4, \frac{2}{7}, \frac{3}{7}, \frac{4}{7}$ \\
\hline \begin{tabular}{|l|}
3 \\
7 \\
\end{tabular} & $3,4, \frac{3}{7}, \frac{4}{7}$ \\
\hline \begin{tabular}{|l|}
4 \\
7 \\
\end{tabular} & $4, \frac{4}{7}$ \\
\hline
\end{tabular}

The comments of this paragraph borrow from Section 4 of [ADLMPW]. In the "coordinates" of semistandard tableaux, the partial ordering and the covering relations in $L_{\mathfrak{g}}^{\beta \alpha}(\lambda)$ are easy to describe. For $\mathbf{s}$ and $\mathbf{t}$ in $L_{\mathfrak{g}}^{\beta \alpha}(\lambda)$, let $S:=\operatorname{tableau}(\mathbf{s})$ and $T:=\operatorname{tableau}(\mathbf{t})$. Then $\mathbf{s} \leq \mathbf{t}$ if and only if $S_{j}^{(i)} \geq T_{j}^{(i)}$ for all $i, j$. (We call this the "reverse componentwise" 
order on tableaux.) Moreover, $\mathbf{s} \rightarrow \mathbf{t}$ is a covering relation in the poset $L_{\mathfrak{g}}^{\beta \alpha}(\lambda)$ if and only if for some $i$ and $j$ we have $S_{j}^{(i)}=T_{j}^{(i)}+1$ while $S_{q}^{(p)}=T_{q}^{(p)}$ for all $(p, q) \neq(i, j)$. For $\mathfrak{g}=A_{1} \times A_{1}$, the edge gets color $\alpha$ if $T_{j}^{(i)}$ is 1 and color $\beta$ if $T_{j}^{(i)}$ is 2 ; for $\mathfrak{g}=A_{2}$, the edge gets color $\alpha$ if $T_{j}^{(i)}$ is 1 and color $\beta$ if $T_{j}^{(i)}$ is 2 ; for $\mathfrak{g}=C_{2}$, the edge gets color $\alpha$ if $T_{j}^{(i)}$ is 1 or 3 and color $\beta$ if $T_{j}^{(i)}$ is 2 ; for $\mathfrak{g}=G_{2}$, the edge gets color $\alpha$ if $T_{j}^{(i)}$ is 1 or 3 or 4 or 6 and color $\beta$ if $T_{j}^{(i)}$ is 2 or 5 . It follows from Proposition 4.7 of [ADLMPW] that for any $\mathbf{t}$ in $L_{\mathfrak{g}}^{\beta \alpha}(\lambda)$, the quantity $w t(\mathbf{t})=\left(m_{\alpha}(\mathbf{t}), m_{\beta}(\mathbf{t})\right)$ coincides with tableauwt $(\operatorname{tableau}(\mathbf{t}))$ for the function tableauwt : $\mathcal{S}_{\mathfrak{g}}(\lambda) \longrightarrow \mathbb{Z} \times \mathbb{Z}$ given by:

$$
\text { tableauwt }(T):= \begin{cases}\left(n_{1}(T)-n_{2}(T)-n_{3}(T), b-2 n_{3}(T)\right) & \text { if } \mathfrak{g}=A_{1} \times A_{1} \\ \left.n_{1}(T)-n_{2}(T), n_{2}(T)-n_{3}(T)\right) & \text { if } \mathfrak{g}=A_{2} \\ \left.n_{1}(T)-n_{2}(T)+n_{3}(T)-n_{4}(T), n_{2}(T)-n_{3}(T)\right) & \text { if } \mathfrak{g}=C_{2} \\ n_{1}(T)-n_{2}(T)+2 n_{3}(T)-2 n_{5}(T)+n_{6}(T)-n_{7}(T), & \\ \left.n_{2}(T)-n_{3}(T)+n_{5}(T)-n_{6}(T)\right) & \text { if } \mathfrak{g}=G_{2}\end{cases}
$$

Here, $n_{k}(T)$ is the number of times the entry $k$ appears in the tableau $T$. Suppose $S=\operatorname{tableau}(\mathbf{s})$ and $T=\operatorname{tableau}(\mathbf{t})$ are $\mathfrak{g}$-semistandard tableaux corresponding to elements $\mathbf{s}$ and $\mathbf{t}$ from $L_{\mathfrak{g}}^{\beta \alpha}(\lambda)$. Then tableau $(\mathbf{s} \vee \mathbf{t})$ (respectively tableau $(\mathbf{s} \wedge \mathbf{t})$ ) is the tableau whose entries are $\min \left(S_{j}^{(i)}, T_{j}^{(i)}\right)$ (respectively $\max \left(S_{j}^{(i)}, T_{j}^{(i)}\right)$ ) for all $i, j$. It now follows that $\operatorname{dist}(\mathbf{s}, \mathbf{t})=\sum_{i, j}\left|S_{j}^{(i)}-T_{j}^{(i)}\right|$. See Figure 6.1 for a $C_{2}$ example.

It is a consequence of Proposition 4.7 that each $\mathfrak{g}$-semistandard lattice is an edgecolored subposet of some supporting graph (namely, the unique maximal support of [Don1]) for the corresponding irreducible representation of $\mathfrak{g}$. Given a $\mathfrak{g}$-semistandard lattice $L$ that is not a supporting graph for $\mathfrak{g}$, we do not know at this time how to find a minimum set of edges to add to $L$ in order to make $L$ a supporting graph for $\mathfrak{g}$. For each semistandard lattice supporting graph from Theorem 5.1, we have explicit formulas for positive rational edge coefficients. For $A_{1} \times A_{1}$, these can be found in [Don1]. For $A_{2}$ $(a \geq 0, b \geq 0), C_{2}(a=0, b \geq 0)$, and $G_{2}(a \geq 0, b=0)$, these can be found in [DLP1]. For the remaining cases of Theorem 5.1, the edge coefficients are explicitly presented in this section. Another way to obtain edge coefficients for $L$ is as follows. First, use the procedure of Remark 4.4 of [DLP2] to obtain the edge product $\pi_{\mathbf{s}, \mathbf{t}}$ on each edge $\mathbf{s} \stackrel{\gamma}{\rightarrow} \mathbf{t}$ in $L$; part (1) of Theorem 4.4 says that the product of coefficients on any edge in $L$ does not depend on the choice of weight basis - in this sense edge products are an invariant of the lattice support $L$. Second, on any edge $\mathbf{s} \stackrel{\gamma}{\rightarrow} \mathbf{t}$ in $L$, set $c_{\mathbf{t}, \mathbf{s}}=d_{\mathbf{s}, \mathbf{t}}:=\sqrt{\pi_{\mathbf{s}, \mathbf{t}}}$. At this point, to see that $L$ together with this assignment of edge coefficients is a representation diagram, one only needs to check diamond relations of type (4) from Section 2. But this follows immediately from the fact that diamond relations of type (5) hold for edge products in $L$. These $x$ - and $y$-coefficients will not always be rational. This procedure for obtaining coefficient products for a given semistandard lattice was implemented in [Alv] using a computer algebra system; the experimental results obtained in small dimensions 
(generally less than 10,000) helped lead us to the coefficient formulas presented here and also lead to the discovery of Propositions 5.7 and 5.8.

Before proceeding with the results needed to prove Theorem 5.1, we record the following corollary of Theorem 5.1. A ranked poset $R$ with rank function $\rho$ and length $l$ is $r a n k$ symmetric if $\left|\rho^{-1}(i)\right|=\left|\rho^{-1}(l-i)\right|$ for $0 \leq i \leq l$. It is rank unimodal if there is an $m$ such that $\left|\rho^{-1}(0)\right| \leq\left|\rho^{-1}(1)\right| \leq \cdots \leq\left|\rho^{-1}(m)\right| \geq\left|\rho^{-1}(m+1)\right| \geq \cdots \geq\left|\rho^{-1}(l)\right|$. It is strongly Sperner if for every $k \geq 1$, the largest union of $k$ antichains is no larger than the largest union of $k$ ranks. It has a symmetric chain decomposition if there exist chains $R_{1}, \ldots, R_{k}$ in $R$ such that (1) as a set $R=R_{1} \cup \cdots \cup R_{k}$ (disjoint union), and (2) for $1 \leq i \leq k$, $\rho\left(\mathbf{y}_{i}\right)+\rho\left(\mathbf{x}_{i}\right)=l$ and $\rho\left(\mathbf{y}_{i}\right)-\rho\left(\mathbf{x}_{i}\right)=l_{i}$, where $\mathbf{x}_{i}$ and $\mathbf{y}_{i}$ are respectively the minimal and maximal elements of the chain $R_{i}$ and $l_{i}$ is the length of the chain $R_{i}$. If $R$ has a symmetric chain decomposition, then one can see that $R$ is rank symmetric, rank unimodal, and strongly Sperner; however, the converse does not hold. Following the discussion in Sections 2 and 5 of [ADLMPW], an edge-colored ranked poset $R$ with edge colors from $\{1, \ldots, n\}$ is a labelling poset for a representation $V$ of a semisimple Lie algebra $\mathfrak{s}$ of rank $n$ if $R$ meets the $\mathfrak{s}$-structure condition and $\left|\left\{\mathbf{t} \in R \mid w t_{R}(\mathbf{t})=\mu\right\}\right|=\operatorname{dim}\left(V_{\mu}\right)$ for each weight $\mu$. Let $z_{1}, \ldots, z_{n}$ be indeterminates, and write $\left(z_{1}, \ldots, z_{n}\right)^{\left(m_{1}, \ldots, m_{n}\right)}$ as shorthand for $z_{1}^{m_{1}} \cdots z_{n}^{m_{n}}$ (in rank two we use the indeterminates $x$ and $y$ ). If $R$ is a labelling poset for an irreducible representation of $\mathfrak{s}$ with highest weight $\lambda$, then one can express the Weyl character $\operatorname{char}_{\mathfrak{s}}\left(\lambda ; z_{1}, \ldots, z_{n}\right):=\sum_{\mu}\left(\operatorname{dim}\left(V_{\mu}\right)\right)\left(z_{1}, \ldots, z_{n}\right)^{\mu}$ as $\sum_{\mathbf{t} \in R}\left(z_{1}, \ldots, z_{n}\right)^{w t_{R}(\mathbf{t})}$.

Corollary 5.3 Let $\lambda=(a, b)$ be a pair of nonnegative integers. Let $L$ be one of the $\mathfrak{g}$ semistandard lattice supporting graphs of Theorem 5.1. Then $L$ is a rank symmetric, rank unimodal, and strongly Sperner poset. Moreover, $L$ is a labelling poset for an irreducible representation of $\mathfrak{g}$ with highest weight $\lambda$, so that

$$
\operatorname{char}_{\mathfrak{g}}(\lambda ; x, y)=\sum_{\mathbf{s} \in L}(x, y)^{w t(\mathbf{s})}
$$

Proof. Since $L$ is a supporting graph for an irreducible representation $V$ of highest weight $\lambda$, it follows from Proposition 2.2 that $L$ meets the structure condition for $\mathfrak{g}$ and that for any weight $\mu$ in $\Lambda,\left|w t^{-1}(\mu)\right|=\operatorname{dim} V_{\mu}$. Then $(L, w t)$ is a labelling poset for $V$, so $\operatorname{char}_{\mathfrak{g}}(\lambda ; x, y)=\sum_{\mathbf{s} \in L}(x, y)^{w t(\mathbf{s})}$. It follows from Proposition 3.11 of [Don1] that $L$ is rank symmetric, rank unimodal, and strongly Sperner.

For the $A_{2}$ semistandard lattices this Sperner result was first proved in $[\mathrm{Pr}]$. For the $C_{2}$ and $G_{2}$ semistandard lattices corresponding to the dominant weights $\lambda=(0, b)$ and $\lambda=(a, 0)$ respectively the above Sperner result follows from [DLP1]. As far as we know it is an open question (aside from some special cases) whether our semistandard lattices have symmetric chain decompositions. A different proof of the remaining assertions of this corollary was given in Section 5 of [ADLMPW].

Consider now the $G_{2}$-fundamental lattice $L_{G_{2}}(0,1)$ of Figure 5.2. Each edge has been assigned two coefficients: the $x$-coefficient is directed up and the $y$-coefficient is directed down. If an edge coefficient is not depicted, it is unity. The next proposition is proved in [Don2]. 
Figure 5.2: The $G_{2}$-fundamental lattice $L_{G_{2}}(0,1)$ as a representation diagram. If an edge coefficient is not depicted, it is unity.

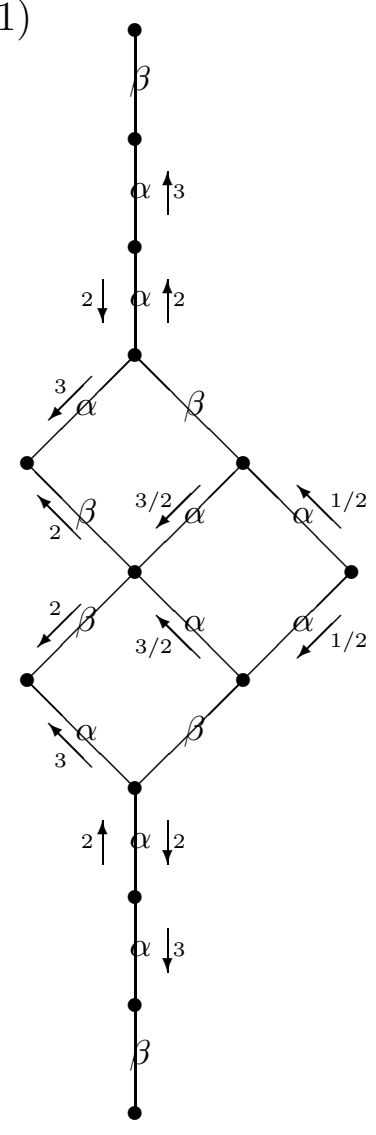

Proposition 5.4 The edge-labelled poset of Figure 5.2 is a representation diagram for an irreducible representation of $G_{2}$ with highest weight $\omega_{\beta}=(0,1)$.

In the language of [DLP1], an irreducible representation of $C_{2}$ of highest weight $\lambda=$ $(a, 0)=a \omega_{\alpha}$ is "one-rowed" since shape $(a, 0)$ is a single row of $a$ boxes. Following Proposition 5.2, a $C_{2}$-semistandard tableau of shape $(a, 0)$ is a filling of $\operatorname{shape}(a, 0)$ with a nondecreasing (reading from left to right) sequence of $a$ integers taken from the set $\{1,2,3,4\}$. (For example, $U=$\begin{tabular}{|l|l|l|l|l|l|}
\hline 1 & 1 & 2 & 2 & 2 & 4 \\
\hline
\end{tabular} shape $(6,0)$ associated to some element $\mathbf{u}$ in $L_{C_{2}}^{\beta \alpha}(6,0)$.) In the spirit of [DLP1], we associate to the $C_{2}$-semistandard tableau $U:=\operatorname{tableau}(\mathbf{u})$ for an element $\mathbf{u}$ in $L_{C_{2}}^{\beta \alpha}(a, 0)$ the 4-tuple onerow $(U)=$\begin{tabular}{|l|l|l|l|}
\hline$u_{1}$ & $u_{2}$ & $u_{3}$ & $u_{4}$ \\
\hline
\end{tabular} obtained by setting $u_{i}:=n_{i}(U)$. (For $U=$ $\operatorname{tableau}(\mathbf{u})$ from our previous example, onerow $(U)=$\begin{tabular}{|l|l|l|l}
\hline 2 & 3 & 0 & 1
\end{tabular} .) Identify the edge color $\alpha$ in $L_{C_{2}}^{\beta \alpha}(a, 0)$ as color 1 , and identify the edge color $\beta$ in $L_{C_{2}}^{\beta \alpha}(a, 0)$ as color 2. By the remarks following Proposition 5.2, one can see then that an edge $\mathbf{s} \stackrel{i}{\rightarrow} \mathbf{t}$ in $L_{C_{2}}^{\beta \alpha}(a, 0)$ can be one of three types:

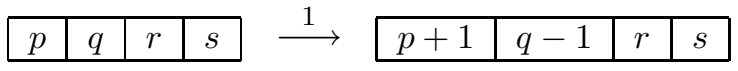




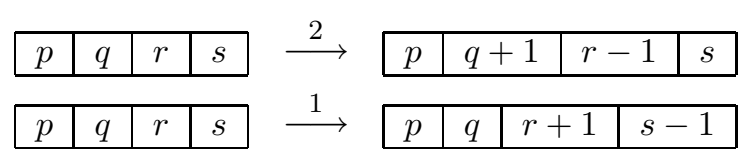

Proposition 5.5 In the notation of the preceding paragraph, let $\mathbf{s} \stackrel{i}{\rightarrow} \mathbf{t}$ be an edge in $L_{C_{2}}^{\beta \alpha}(a, 0)$. Attach coefficients $c_{\mathbf{t}, \mathbf{s}}$ and $d_{\mathbf{s}, \mathbf{t}}$ to the edge $\mathbf{s} \stackrel{i}{\rightarrow} \mathbf{t}$ according to Figure 5.3. With this assignment of edge coefficients, the edge-labelled poset $L_{C_{2}}^{\beta \alpha}(a, 0)$ is a representation diagram for $C_{2}$. In particular, $L_{C_{2}}^{\beta \alpha}(a, 0)$ is a positive integral supporting graph for an irreducible representation of $C_{2}$ of highest weight $(a, 0)=a \omega_{\alpha}$.

Figure 5.3: Coefficients for Proposition 5.5.

\begin{tabular}{|c|c|}
\hline Edge $\mathbf{s} \stackrel{i}{\longrightarrow} \mathbf{t}$ in $L_{C_{2}}^{\beta \alpha}(a, 0)$ & Edge Coefficients \\
\hline 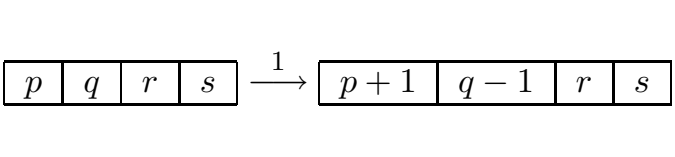 & $\begin{array}{c}c_{\mathbf{t}, \mathbf{s}}=p+1 \\
d_{\mathbf{s}, \mathbf{t}}=q\end{array}$ \\
\hline \begin{tabular}{|l|l|l|l|l|l|l|l|}
$p$ & $q$ & $r$ & $s$ \\
\end{tabular}$\stackrel{2}{\longrightarrow}$\begin{tabular}{|l|l|l|l|l|} 
& $q+1$ & $r-1$ & $s$ \\
\end{tabular} & $\begin{array}{c}c_{\mathbf{t}, \mathbf{s}}=q+1 \\
d_{\mathbf{s}, \mathbf{t}}=r\end{array}$ \\
\hline \begin{tabular}{|l|l|l|l|l|l|l|l|l|}
$p$ & $q$ & $r$ & $s$ \\
\end{tabular}$\stackrel{1}{\longrightarrow}$\begin{tabular}{|l|l|l|l|l|} 
& $q$ & $r+1$ & $s-1$ \\
\end{tabular} & $\begin{array}{c}c_{\mathbf{t}, \mathbf{s}}=r+1 \\
d_{\mathbf{s}, \mathbf{t}}=s\end{array}$ \\
\hline
\end{tabular}

In algebraic terms, in the proof that follows we are regarding $C_{2}$ as a subalgebra of $A_{3}$; it turns out that when an irreducible $A_{3}$-module of highest weight $a \omega_{1}$ is viewed as a $C_{2}$-module under the induced action, it remains irreducible.

Proof. By inspection, the coefficients in Figure 5.3 are positive integers. By Proposition 4.3, it follows that if $L_{C_{2}}^{\beta \alpha}(a, 0)$ is a supporting graph for $C_{2}$, then it is a supporting graph for an irreducible representation of $C_{2}$ of highest weight $(a, 0)=a \omega_{\alpha}$. To complete the proof we apply Proposition 2.2: we show that the edge-labelled poset $L_{C_{2}}^{\beta \alpha}(a, 0)$ is a representation diagram by verifying the diamond, crossing, and $C_{2}$-structure conditions. By Proposition 4.1, $L_{C_{2}}^{\beta \alpha}(a, 0)$ meets the structure condition for $C_{2}$. It remains to show that the edge-labelled poset $L_{C_{2}}^{\beta \alpha}(a, 0)$ with coefficients taken from Figure 5.3 satisfies the diamond and crossing conditions. The $C_{2}$-semistandard tableaux of shape $(a, 0)$ are exactly the same as the tableaux of shape $\square[\cdots] \square$ (there are $a$ boxes here) used to build the $A_{3}$ 
lattice denoted $L_{A}^{G T-\text { left }}(3, \square \square \cdots \square \square)$ in Section 4 of [Don1]. One can see that $L_{C_{2}}^{\beta \alpha}(a, 0)$ and $L_{A}^{G T-l e f t}(3, \square \square \cdots \square \square)$ are isomorphic as lattices, but with slightly different edge coloring schemes. For the remainder of this proof, set $L_{A}^{G T-l e f t}:=L_{A}^{G T-l e f t}(3, \square \square \cdots \square \square)$. In [Don1], edges of the $A_{3}$ lattice $L_{A}^{G T-l e f t}$ are colored 1,2 , or 3 : the edge $S \longrightarrow T$ is given color $i$ if an entry $i$ in the tableau $T$ changes to $i+1$ to form the tableau $S$. It is easy to see that all $i$-components in $L_{A}^{G T \text {-left }}$ are chains. Pick an $i$-component $\mathcal{C}$ in $L_{A}^{G T-l e f t}$ and supply the edges of this chain with coefficients as follows: If $S \stackrel{i}{\longrightarrow} T$ is an edge in $\mathcal{C}$, set $c_{T, S}=\rho_{i}(T)$ and $d_{S, T}=l_{i}(T)-\rho_{i}(T)+1$. It now follows from Theorem 6.4 of [Don1] that with this assignment of edge coefficients, $L_{A}^{G T-l e f t}$ is a representation diagram for $A_{3}$ and therefore meets the diamond and crossing conditions. (In the language of that paper, $L_{A}^{G T-l e f t}$ is now a representation diagram for a "one-dimensional weight space representation" of $A_{3}$.) For $1 \leq i \leq 3$ and for a tableau $T$ in $L_{A}^{G T-l e f t}$, one can easily see that we have $\rho_{i}(T)=n_{i}(T)$ and $l_{i}(T)-\rho_{i}(T)+1=n_{i+1}(T)-1$. Now change all edges of color 3 in $L_{A}^{G T \text {-left }}$ to color 1 to obtain the $C_{2}$-semistandard lattice $L_{C_{2}}^{\beta \alpha}(a, 0)$. Observe now that the coefficients on the edges of $L_{A}^{G T-l e f t}$ coincide with the edge coefficients for $L_{C_{2}}^{\beta \alpha}(a, 0)$ supplied by Figure 5.3. Thus this assignment of coefficients to the edges of $L_{C_{2}}^{\beta \alpha}(a, 0)$ will satisfy the diamond condition at any diamond and will satisfy the crossing condition for color 2 because the 2-components of $L_{C_{2}}^{\beta \alpha}(a, 0)$ are the same as the 2-components of $L_{A}^{G T-l e f t}$.

We now only need to check the crossing condition for color 1 in the $C_{2}$-semistandard lattice $L_{C_{2}}^{\beta \alpha}(a, 0)$. The color 1 component of a tableau $T$ in $L_{C_{2}}^{\beta \alpha}(a, 0)$ coincides with the $\{1,3\}$-component of $T$ in $L_{A}^{G T-l e f t}$. For the moment let us work inside of $L_{A}^{G T-l e f t}$. Let $k:=l_{1}(T)$ and $i:=\rho_{1}(T)$ (the length of the 1-component of $T$ in $L_{A}^{G T-l e f t}$ and the rank of $T$ in the 1-component, respectively); similarly let $l:=l_{3}(T)$ and $j:=\rho_{3}(T)$. One can check that the color 1 edge below $T$ (if it exists) has product $(k-i+1)(i)$, the color 3 edge below $T$ (if it exists) has product $(l-j+1)(j)$, the color 1 edge above $T$ (if it exists) has product $(k-i)(i+1)$, and the color 3 edge above $T$ (if it exists) has product $(l-j)(j+1)$. Now change color 3 to color 1 . It is clear that the length of the 1-component containing $T$ in $L_{C_{2}}^{\beta \alpha}(a, 0)$ is $k+l$, and that the rank of $T$ in this 1 -component is $i+j$. To check the crossing condition for color 1 at the vertex $T$ in $L_{C_{2}}^{\beta \alpha}(a, 0)$ now requires us simply to verify the following identity, which can be done by inspection:

$$
(l-j+1)(j)+(k-i+1)(i)-(k-i)(i+1)-(l-j)(j+1)=2 i+2 j-l-k .
$$

By Lemma 4.6, Proposition 5.6 below accounts for two positive rational weight bases for an irreducible representation of $C_{2}$ with highest weight $\lambda=(1, b)$ with $b \geq 1$, one each for $L_{C_{2}}^{\beta \alpha}(\lambda)$ and $L_{C_{2}}^{\alpha \beta}(\lambda)$; the two bases are distinct since, by Corollary 4.5 , two weight bases with supporting graphs $L_{C_{2}}^{\beta \alpha}(\lambda)$ and $L_{C_{2}}^{\alpha \beta}(\lambda)$ respectively will not be diagonally equivalent. For $\mathbf{u}$ in $L_{C_{2}}^{\beta \alpha}(\lambda)$, the tableau $U:=\operatorname{tableau}(\mathbf{u})$ has two parts: the first $b$ columns $\left(U^{(1)}, \ldots, U^{(b)}\right)$ of $U$ which are all columns of length two and together form a tableau $U^{\prime} \in L_{C_{2}}^{\beta \alpha}(0, b)$ (we call this the two-row part of $U$ or $\mathbf{u}$ ), and the last column $U^{(b+1)}$ of $U$ which is a column consisting of a single box (we call this the one-row part of $U$ or $\mathbf{u}$ ). Associate to $U^{(b+1)}$ the 4-tuple onerow $\left(U^{(b+1)}\right)=$\begin{tabular}{|l|l|l|l|}
\hline$u_{1}$ & $u_{2}$ & $u_{3}$ & $u_{4}$ \\
\hline
\end{tabular} (note that each $u_{i} \in\{0,1\}$ 
and $\left.\sum u_{i}=1\right)$, and associate to the tableau $U^{\prime}$ the 5-tuple tworow $\left(U^{\prime}\right):=$\begin{tabular}{|l|l|}
\hline$u_{1}^{\prime}$ & $u_{2}^{\prime}$ \\
\hline$u_{5}^{\prime}$ & $u_{4}^{\prime}$ \\
\hline
\end{tabular} where $u_{1}^{\prime}$ counts the number of columns of the form $\frac{1}{2}$ in $U^{\prime}, u_{2}^{\prime}$ counts the number of columns of the form $\frac{1}{3}$ in $U^{\prime}, u_{3}^{\prime}$ counts the number of columns of the form $\frac{2}{3}$ in $U, u_{4}^{\prime}$ counts the number of columns of the form $\frac{2}{4}$ in $U$, and $u_{5}^{\prime}$ counts the number of columns of the form $\frac{3}{4}$ in $U^{\prime}$. Identify the edge color $\alpha$ in $L_{C_{2}}^{\beta \alpha}(\lambda)$ as color 1 , and identify the edge color $\beta$ in $L_{C_{2}}^{\beta \alpha}(\lambda)$ as color 2. By the remarks immediately following Proposition 5.2, one can see then that an edge $\mathbf{s} \stackrel{i}{\rightarrow} \mathbf{t}$ in $L_{C_{2}}^{\beta \alpha}(\lambda)$ can be one of the following seven types; the first four of these types of edges have changes occurring in the two-row parts of the tableaux, and the last three types of edges have changes occurring in the one-row parts.

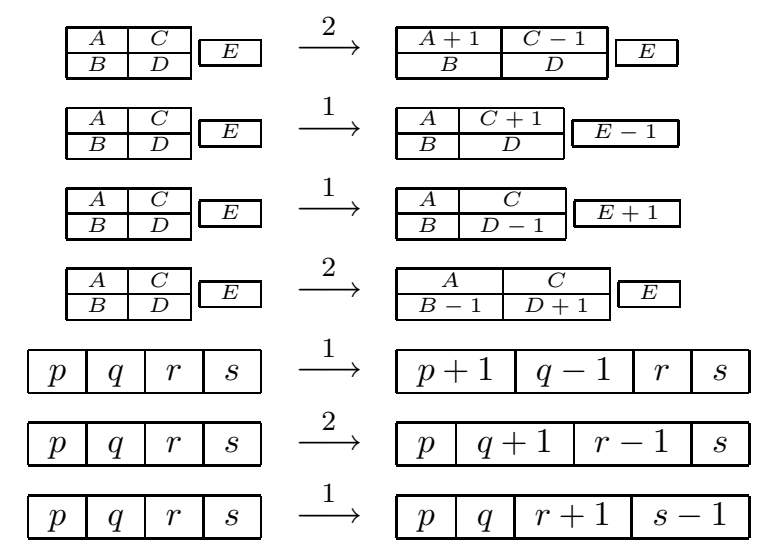

Since $p, q, r$, and $s$ are nonnegative integers whose sum is one, the last three of these edge types can also be characterized respectively as follows:

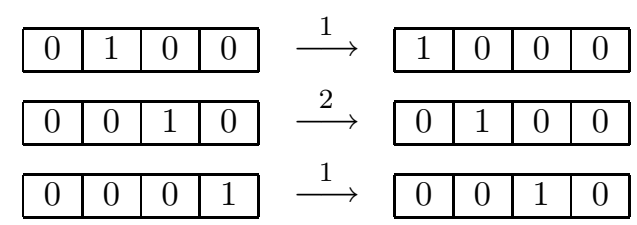

Proposition 5.6 Let $b$ be a nonnegative integer, and let $\lambda=(1, b)$. Keeping the notation of the previous paragraph, let $\mathbf{s} \stackrel{i}{\rightarrow} \mathbf{t}$ be an edge in $L_{C_{2}}^{\beta \alpha}(\lambda)$, where \begin{tabular}{l|l|l|}
\hline$A$ & $C$ \\
\hline$B$ & $D$ \\
\hline
\end{tabular} corresponds to the two-row part of $\operatorname{tableau(s)}$ and \begin{tabular}{|l|l|l|l|}
\hline$p$ & $q$ & $r$ & $s$ \\
\hline
\end{tabular} Attach coefficients $c_{\mathbf{t}, \mathbf{s}}$ and $d_{\mathbf{s}, \mathbf{t}}$ to the edge $\mathbf{s} \stackrel{i}{\rightarrow} \mathbf{t}$ according to Figure 5.4. With this assignment of edge coefficients, the edge-labelled poset $L_{C_{2}}^{\beta \alpha}(\lambda)$ is a representation diagram for $C_{2}$. In particular, $L_{C_{2}}^{\beta \alpha}(\lambda)$ is a positive rational supporting graph for an irreducible representation of $C_{2}$ of highest weight $\lambda$.

Proof. By inspection, the coefficients in Figure 5.4 are positive rational numbers. Let $L:=L_{C_{2}}^{\beta \alpha}(1, b)$. By Proposition 4.3, it follows that if $L$ is a supporting graph for $C_{2}$, then it is a supporting graph for an irreducible representation of $C_{2}$ of highest weight $(1, b)=\omega_{\alpha}+b \omega_{\beta}$. To complete the proof we apply Proposition 2.2: we show that the edge-labelled poset $L$ is a representation diagram by verifying the diamond, crossing, and 
$C_{2}$-structure conditions. By Proposition $4.1, L$ meets the structure condition for $C_{2}$. It remains to show that the edge-labelled poset $L$ with coefficients taken from Figure 5.4 satisfies the diamond and crossing conditions.

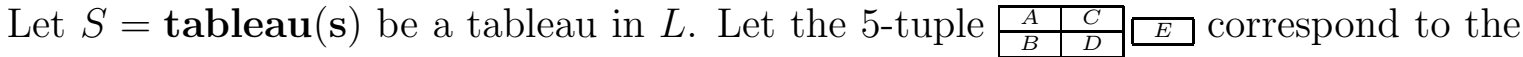
two-row part of $S$ as in the paragraph preceding the proposition statement, and let the

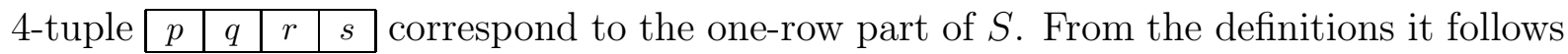
that tableauwt $(S)=(2 C-2 D+p-q+r-s, A-C+D-B+q-r)$. From Proposition 4.7 of $[$ ADLMPW] we see then that $w t(\mathbf{s})=(2 C-2 D+p-q+r-s, A-C+D-B+q-r)$. Figure 5.5 depicts all possible ancestors and descendants of $S$ along edges of color 1 in $L$. Similarly, Figure 5.6 depicts all possible ancestors and descendants of $S$ along edges of color 2 . To check the crossing condition at $S$ for color 1, we consider the following seven cases: $E=0$ and $p=1 ; E=0$ and $q=1 ; E=0$ and $r=1 ; E=0$ and $s=1 ; E=1$ and $q=1 ; E=1$ and $r=1 ; E=1$ and $s=1$. Since $E$ counts the number of columns of the form $\frac{2}{3}$ in $S$, then $E$ must be zero or one. Moreover, we cannot simultaneously have $E=1$ and $p=1$. Also, $p, q, r$, and $s$ are each zero or one, and at most one of them can be nonzero. Hence the above seven cases account for all possibilities for the tableau $S$. As an example, when $E=0$ and $q=1$, note that $2 C-2 D+p-q+r-s=2 C-2 D-1$. Using Figure 5.4 we see that the sum of the edge products for edges below $S$ minus the sum of the edge products for edges above $S$ is:

$$
\begin{aligned}
\left\{\begin{aligned}
\frac{(2 D+1)(2 D+2)(2 C)}{2 D+1}-\frac{2 D(2 C+1)(2 D+1)}{2 D} & \text { if } D>0 \\
\frac{(2 D+1)(2 D+2)(2 C)}{2 D+1}-(2 C+1) & \text { if } D=0
\end{aligned}\right. \\
= \begin{cases}(2 D+2)(2 C)-(2 C+1)(2 D+1) & \text { if } D>0 \\
(2)(2 C)-(2 C+1) & \text { if } D=0\end{cases} \\
= \begin{cases}2 C-2 D-1 & \text { if } D>0 \\
2 C-1 & \text { if } D=0,\end{cases}
\end{aligned}
$$

which in either case is $2 C-2 D-1$. We have similarly verified the identities for the remaining six cases. To check the crossing condition for color 2 at $S$, we consider the following six cases: $B=0$ and $p=1 ; B=0$ and $q=1 ; B=0$ and $r=1 ; B=0$ and $s=1 ; B>0$ and $r=1 ; B>0$ and $s=1$. Note that $B$ counts the number of columns of the form $\frac{3}{4}$ in $S$, so if $B>0$, then $p=q=0$. Hence the above six cases account for all possibilities for the tableau $S$. We have verified the crossing relation identities that result in each of these six cases in a fashion similar to the above.

For the diamond condition, we begin by noting as in the previous paragraph that for the tableau $S$, only one of the following is true: $E=0$ and $p=1 ; E=0$ and $q=1$; $E=0$ and $r=1 ; E=0$ and $s=1 ; E=1$ and $q=1 ; E=1$ and $r=1 ; E=1$ and $s=1$. These conditions identify seven classes of tableaux in $L$. If $S$ is in a given class, we will consider all possible ways to build a diamond $T \dot{\not}_{R}^{\dot{\chi}_{R}} S$. When $E=0$ and 
$p=1$, there is only one possible way to build such a diamond, and this requires $C>0$ :

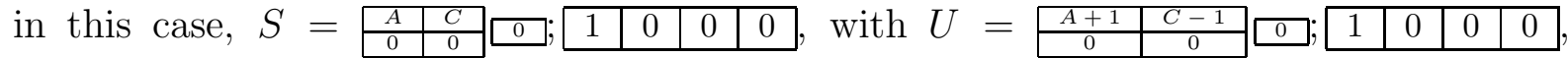

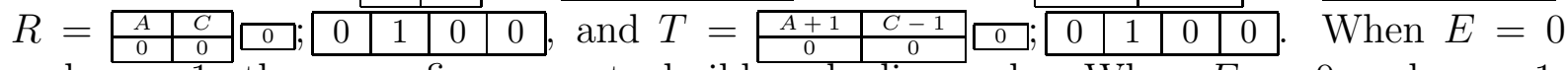
and $q=1$, there are five ways to build such diamonds. When $E=0$ and $r=1$, there are eleven ways to build such diamonds. When $E=0$ and $s=1$, there are nine ways to build such diamonds. When $E=1$ and $q=1$, there are four ways to build such diamonds. When $E=1$ and $r=1$, there are eleven ways to build such diamonds. When $E=1$ and $s=1$, there are nine ways to build such diamonds. In total, 50 such diamonds are possible. For example, when $E=0$ and

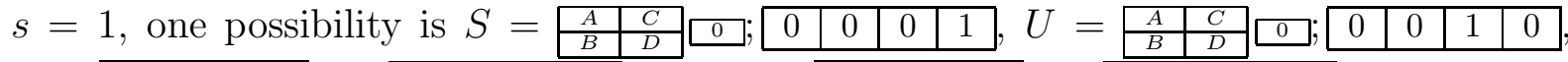

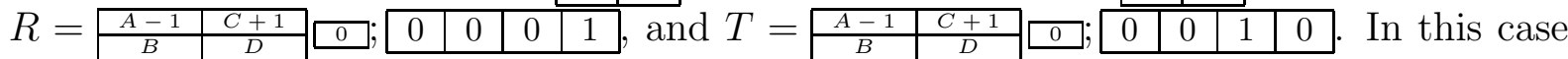
use Figure 5.4 to check that $c_{U, S}=(B+1)(2 B+2 C+2 D+3), d_{T, U}=\frac{A(2 C+1)(2 B+2 C+2 D+5)}{(2 C+2 D+3)(2 B+2 C+2 D+3)}$, $d_{R, S}=\frac{A(2 C+1)}{2 C+2 D+3}$, and $c_{T, R}=(B+1)(2 B+2 C+2 D+5)$. Hence, $c_{U, S} d_{T, U}=d_{R, S} c_{T, R}$, which confirms the diamond relation in this case. We have similarly verified the diamond condition for the remaining cases.

Proposition 5.7 Let $\lambda=(a, b)$ for integers $a$ and $b$ with $a \geq 2$ and $b \geq 1$. Then the $C_{2}$-semistandard lattices $L_{C_{2}}^{\beta \alpha}(\lambda)$ and $L_{C_{2}}^{\alpha \beta}(\lambda)$ are not supporting graphs for $C_{2}$.

Proof. We will assume first that $a \geq 4$ and $b \geq 4$. We proceed by contradiction and suppose that for some set of edge coefficients, $L_{C_{2}}^{\beta \alpha}(\lambda)$ is a supporting graph for a representation of $C_{2}$. Now $l=\left|P_{C_{2}}^{\beta \alpha}(\lambda)\right|$ is also the length of $L_{C_{2}}^{\beta \alpha}(\lambda)$. Let $Q_{\lambda}$ be the subposet of all vertices $\mathbf{t}$ of $L_{C_{2}}^{\beta \alpha}(\lambda)$ for which $0 \leq l-\rho(\mathbf{t}) \leq 4$ together with the partial ordering on these elements induced by $L_{C_{2}}^{\beta \alpha}(\lambda)$. Let each edge of the Hasse diagram for $Q_{\lambda}$ have the same color as the corresponding edge in $L_{C_{2}}^{\beta \alpha}(\lambda)$. We will use the $C_{2}$-semistandard tableaux described in Proposition 5.2 for the remainder of this argument. Relative to the total ordering $\left(\mathbf{t}_{0}, \mathbf{t}_{1}, \mathbf{t}_{2}, \ldots\right)$ on elements of $L_{C_{2}}^{\beta \alpha}(\lambda)$, let $T_{i}:=\operatorname{tableau}\left(\mathbf{t}_{i}\right)$. Let $\mu:=(4,4)$. Observe that $Q_{\lambda}$ and $Q_{\mu}$ are isomorphic as edge-colored posets. To see this, note that if a tableau $T$ is within 4 steps of the maximal tableau, then it differs from the maximal tableau in at most 4 different columns. Thus the first $b-4$ columns of $T$ will be $\frac{1}{2}$, s, while $T^{(b+1)}=T^{(b+2)}=\ldots=T^{(a+b-4)}=1$. So we can identify the tableau $T$ in $Q_{\lambda}$ with the tableau $\left(T^{(b-3)}, T^{(b-2)}, T^{(b-1)}, T^{(b)}, T^{(a+b-3)}, T^{(a+b-2)}, T^{(a+b-1)}, T^{(a+b)}\right) \in Q_{\mu}$.

We depict a generic $Q_{\lambda}$ in Figure 5.7. In this figure, the color 1 edges correspond to color $\alpha$ while the color 2 edges correspond to color $\beta$. In Figure 5.9, we list the 25 vertices of $Q_{\lambda}$, record the distance of each vertex from the corresponding boundary element, and compute the weight of each vertex (thought of now as an element of $L_{C_{2}}^{\beta \alpha}(\lambda)$ ). We used the function tableauwt and Proposition 4.7 from [ADLMPW] to aid in the computations of Figure 5.9. Using this data, we will proceed as in the proof of Theorem 4.1 of [DLP1] to compute the edge products. We need not assume these edge products are nonzero; this will follow from the formulas we derive for each of the edge products that are relevant for our argument. We start with those edges below the maximal vertex $\mathbf{t}_{0}$, then those edges below $\mathbf{t}_{1}$, etc. We use an appropriate diamond or crossing relation at each edge. The coefficient products for the edges below vertices $\mathbf{t}_{0}$ through $\mathbf{t}_{11}$ are: $\pi_{1,0}=b, \pi_{2,0}=a, \pi_{3,1}=2(b-1)$, 


$$
\begin{aligned}
& \pi_{4,1}=a+2, \pi_{4,2}=\frac{a b}{a+2}, \pi_{5,2}=2(a-1), \pi_{6,2}=\frac{a+2 b+2}{a+2}, \pi_{7,3}=3(b-2), \pi_{8,3}=a+4, \\
& \pi_{8,4}=\frac{2(a+2)(b-1)}{a+4}, \pi_{9,4}=2(a+1), \pi_{10,4}=\frac{a(a+2 b+2)}{(a+2)(a+4)}, \pi_{9,5}=\frac{a b(a-1)}{(a+1)(a+2)}, \pi_{11,5}=3(a-2), \\
& \pi_{12,5}=\frac{2\left(2 a b+b+a^{2}+3 a+2\right)}{(a+1)(a+2)}, \pi_{10,6}=\frac{b(a+4)}{a+2}, \pi_{12,6}=\frac{(a-1)(a+1)(a+2 b+2)}{2 a b+b+a^{2}+3 a+2}, \pi_{13,6}=\frac{(a+2)(a+b+1)}{2 a b+b+a^{2}+3 a+2}, \\
& \pi_{14,7}=4(b-3), \pi_{15,7}=a+6, \pi_{15,8}=\frac{3(a+4)(b-2)}{a+6}, \pi_{16,8}=2(a+3), \pi_{17,8}=\frac{a(a+2 b+2)}{(a+6)(a+4)}, \pi_{16,9}= \\
& \frac{2(a+1)(a+2)(b-1)}{(a+3)(a+4)}, \pi_{18,9}=3 a, \pi_{19,9}=\frac{2\left(2 a^{3} b+7 a^{2} b+7 a b+8 b+a^{4}+6 a^{3}+13 a^{2}+12 a+4\right)}{(a+1)(a+2)(a+3)(a+4)}, \pi_{17,10}=\frac{2(a+6)(b-1)}{a+4}, \\
& \pi_{19,10}=\frac{a(a+1)}{2 a^{3} b+7 a^{2} b+7 a b+8 b+a^{4}+6 a^{3}+13 a^{2}+12 a+4}, \quad \pi_{20,10}=\frac{(a+4)\left(a^{3}+a^{2} b+4 a^{2}+3 a b+5 a+4 b+2\right)}{2 a^{3} b+7 a^{2} b+7 a b+8 b+a^{4}+6 a^{3}+13 a^{2}+12 a+4}, \\
& \pi_{18,11}=\frac{b(a-1)(a-2)}{(a+1)(a+2)}, \pi_{21,11}=4(a-3), \text { and } \pi_{22,11}=\frac{3\left(2 a b+a^{2}+3 a+2\right)}{(a+1)(a+2)} . \text { One can now check that } \\
& \text { at vertex } \mathbf{t}_{12}, \text { the diamond relation } \pi_{9,5} \pi_{12,5}=\pi_{19,9} \pi_{19,12} \text { returns }
\end{aligned}
$$

$$
\pi_{19,12}=\frac{a b(a-1)\left(2 a b+b+a^{2}+3 a+2\right)(a+3)(a+4)}{\left(2 a^{3} b+7 a^{2} b+7 a b+8 b+a^{4}+6 a^{3}+13 a^{2}+12 a+4\right)(a+1)(a+2)}
$$

while the diamond relation $\pi_{10,6} \pi_{12,6}=\pi_{19,10} \pi_{19,12}$ returns

$$
\pi_{19,12}=\frac{b(a+4)(a-1)\left(2 a^{3} b+7 a^{2} b+7 a b+8 b+a^{4}+6 a^{3}+13 a^{2}+12 a+4\right)}{a(a+1)(a+2)(a+3)\left(2 a b+b+a^{2}+3 a+2\right)} .
$$

It is easy to check that for a pair of positive integers $a$ and $b$, these two expressions for $\pi_{19,12}$ will be the same only if $a=1$. Thus no set of edge coefficients for $L_{C_{2}}^{\beta \alpha}(\lambda)$ will satisfy all diamond and crossing relations, which contradicts our hypothesis that $L_{C_{2}}^{\beta \alpha}(\lambda)$ is a supporting graph for $C_{2}$. It now follows from Lemma 4.6 that for such $\lambda, L_{C_{2}}^{\alpha \beta}(\lambda)$ is not a supporting graph for $C_{2}$.

The remaining cases $(a \in\{2,3\}$ and $b \geq 1 ; b \in\{2,3\}$ and $a \geq 2$ ) can be viewed as special cases of the preceding argument. For example, when $a=3$ and $b \geq 4, Q_{\lambda}$ will be the same as depicted in Figure 5.7, with the exception that vertex $T_{21}=\operatorname{tableau}\left(\mathbf{t}_{21}\right)$ is not present and hence $\mathbf{t}_{21} \rightarrow \mathbf{t}_{11}$ is not an edge in $L_{C_{2}}^{\beta \alpha}(\lambda)$. The data from Figure 5.9 is still valid if we evaluate each expression at $a=3$ and omit the data for $T_{21}$. Note that when $a=3$, the above formula for $\pi_{21,11}$ returns a value of zero, which is consistent with our observation that this edge is not present in $L_{C_{2}}^{\beta \alpha}(\lambda)$. At this point one can check that the formulas obtained above for the other coefficient products are valid at $a=3$ and can be computed in the same order. This sequence of computations will lead to the same inconsistency encountered at the end of the previous paragraph on edge $\mathbf{t}_{19} \rightarrow \mathbf{t}_{12}$ when we applied two different diamond relations to obtain two distinct values for $\pi_{19,12}$.

Proposition 5.8 Let $\lambda=(a, b)$ for integers $a$ and $b$ with $a \geq 1, b \geq 1$ or $a=0, b \geq 2$. Then the $G_{2}$-semistandard lattices $L_{G_{2}}^{\beta \alpha}(\lambda)$ and $L_{G_{2}}^{\alpha \beta}(\lambda)$ are not supporting graphs for $G_{2}$.

Proof. This is very similar to the proof of Proposition 5.7. When $a \geq 4$ and $b \geq 4$, the " $Q_{\lambda}$ " portion of the $G_{2}$-semistandard lattice is the same as in the $C_{2}$ case (depicted in Figure 5.7) but with an additional color 1 edge $\mathbf{t}_{25} \stackrel{1}{\rightarrow} \mathbf{t}_{13}$. This is depicted in Figure 5.8. Note that within four steps of the maximal element, the $C_{2}$ and $G_{2}$ restrictions on tableaux nearly coincide. The $G_{2}$ data of Figure 5.9 does differ from the $C_{2}$ data; however, the coefficient products $\pi_{1,0}, \ldots, \pi_{22,11}$ for $L_{G_{2}}^{\beta \alpha}(\lambda)$ can be computed in the same order as in the $C_{2}$ case. This leads to an inconsistency on edge $\mathbf{t}_{19} \rightarrow \mathbf{t}_{12}$ similar to the one encountered 
in the $C_{2}$ case. Analysis of the special cases $a \in\{2,3\}, b \geq 1$ or $b \in\{2,3\}, a \geq 2$ will be similar to the corresponding $C_{2}$ special cases. The cases $a=0, b \geq 2$ and $a=1, b \geq 2$ require a few more adjustments. In these cases the inconsistency occurs on an edge $\mathbf{s} \rightarrow \mathbf{t}$ with $l-\rho(\mathbf{t})=5$. The inconsistency for the 64-dimensional $a=1, b=1$ case can be found by hand.

Proof of Theorem 5.1. In all cases it suffices to prove the results for $L_{\mathfrak{g}}^{\beta \alpha}(\lambda)$; the results for $L_{\mathfrak{g}}^{\alpha \beta}(\lambda)$ then follow from Lemma 4.6. From Proposition 4.3 and Theorem 4.4 it follows that if $L_{\mathfrak{g}}^{\beta \alpha}(\lambda)$ is a supporting graph for a representation of $\mathfrak{g}$, then the representation is irreducible with highest weight $\lambda$ and $L_{\mathfrak{g}}^{\beta \alpha}(\lambda)$ is solitary and edge-minimal. Some of the $C_{2}$ and $G_{2}$ cases are handled by Propositions 5.4, 5.5, 5.6, 5.7, and 5.8. Now we consider the remaining cases.

Let $\mathfrak{g}=A_{1} \times A_{1}$. As noted prior to the statement of Lemma 4.3 of [ADLMPW], $L_{A_{1} \times A_{1}}^{\beta \alpha}(\lambda)$ and $L_{A_{1} \times A_{1}}^{\alpha \beta}(\lambda)$ are edge-color isomorphic to $L_{A_{1} \times A_{1}}^{\beta \alpha}(a, 0) \times L_{A_{1} \times A_{1}}^{\beta \alpha}(0, b)$. Observe that $L_{A_{1} \times A_{1}}^{\beta \alpha}(a, 0)$ is a chain of length $a$ with all edges of color $\alpha$ and that $L_{A_{1} \times A_{1}}^{\beta \alpha}(0, b)$ is a chain of length $b$ with all edges of color $\beta$. By Section 3.4 of [Don1], $L_{A_{1} \times A_{1}}^{\beta \alpha}(a, 0)$ and $L_{A_{1} \times A_{1}}^{\beta \alpha}(0, b)$ are supporting graphs for irreducible one-dimensional weight space representations of the respective copies of $A_{1}$ that form the product $A_{1} \times A_{1}$. By arguments preceding the proof of Corollary 6.8 of that paper, the irreducible representation of $A_{1} \times A_{1}$ with highest weight $\lambda$ is a one-dimensional weight space representation. As observed in the proof of Corollary 6.8 there, Lemma 3.3.B of [Don1] shows that its unique (up to edge-colored poset isomorphism) supporting graph is the edge-colored distributive lattice $L_{A_{1} \times A_{1}}^{\beta \alpha}(a, 0) \times L_{A_{1} \times A_{1}}^{\beta \alpha}(0, b)$. Now use Theorem 6.4 of [Don1] to see that the supporting graph $L_{A_{1} \times A_{1}}^{\beta \alpha}(a, 0) \times L_{A_{1} \times A_{1}}^{\beta \alpha}(0, b)$ for $A_{1} \times A_{1}$ is positive integral.

For $\mathfrak{g}=A_{2}$, see Proposition 4.1 of [Don1]. There, a construction originally due to Gel'fand and Tsetlin [GT] is used to obtain two positive rational weight bases (respectively, the "GT-left" and "GT-right" bases) for the irreducible representation of $A_{2}$ with dominant weight $\lambda$. The positive rational supporting graph for the GT-left basis is denoted $L_{A}^{G T-l e f t}(2, \lambda)$. Using the description of elements of $L_{A_{2}}^{\beta \alpha}(\lambda)$ as $A_{2}$-semistandard tableaux, it is immediate that $L_{A}^{G T-l e f t}(2, \lambda) \cong L_{A_{2}}^{\beta \alpha}(\lambda)$, an isomorphism of edge-colored distributive lattices where edge color $\alpha$ (respectively $\beta$ ) in $L_{A_{2}}^{\beta \alpha}(\lambda)$ is identified with color 1 (respectively 2). In [Don1] one sees that the positive rational supporting graph $L_{A}^{G T-r i g h t}(2, \lambda)$ for the GT-right basis is, in the notation of this paper, simply $L_{A}^{G T-l e f t}(2, \lambda)^{\triangle}$. Then $L_{A}^{G T-\text { right }}(2, \lambda) \cong L_{A_{2}}^{\alpha \beta}(\lambda)$. The GT-left basis was re-derived in [DLP1] as follows. Consider the irreducible $B_{3}$-module realized by the representation diagram $L_{B}^{\mathrm{RS}}(k, 6)$ where $k=a+b$; its edge colors are from the set $\{1,2,3\}$. The component $\operatorname{comp}_{\{1,2\}}(\mathbf{t})$ containing the element $\mathbf{t}=$\begin{tabular}{|l|l|l}
$a$ & 0 & 0 \\
\hline 0 & 0 & $b$ \\
\hline
\end{tabular} is isomorphic as an edge-colored distributive lattice to $L_{A}^{G T-l e f t}(2, \lambda)$. With respect to this identification, supply the edges of $L_{A}^{G T-l e f t}(2, \lambda)$ with the positive rational edge-coefficients of Theorem 1.2.RS from [DLP1]; then $L_{A}^{G T-l e f t}(2, \lambda)$ is a representation diagram for an irreducible $A_{2}$-module of highest weight $\lambda$.

Now let $\mathfrak{g}=C_{2}$ and $\lambda=(0, b)$. In this case, the $C_{2}$-semistandard lattices $L_{C_{2}}^{\beta \alpha}(\lambda)$ and $L_{C_{2}}^{\alpha \beta}(\lambda)$ are the same. In what follows, identify the color $\alpha$ edges of the $C_{2}$-semistandard lattice with the color 1 , and identify the color $\beta$ edges with color 2 . The $C_{2}$-semistandard 
lattice $L_{C_{2}}^{\beta \alpha}(\lambda)$ is just a recoloring of the Molev lattice $L_{B}^{M o l}(b, 4)$ from [DLP1]. To see this, observe that the 5 -tuple tworow $(T)=$\begin{tabular}{|l|l|l}
\hline$t_{1}$ & $t_{2}$ \\
\hline$t_{5}$ & $t_{4}$ \\
\hline$t_{3}$
\end{tabular} corresponding to a $C_{2}$-semistandard tableau $T=\left(T^{(1)}, \ldots, T^{(b)}\right)$ in $L_{C_{2}}^{\beta \alpha}(\lambda)$ is an element in $L_{B}^{M o l}(b, 4)$. With the help of the remarks following Proposition 5.2, one can check that (ignoring colors) $S \longrightarrow T$ in $L_{C_{2}}^{\beta \alpha}(\lambda)$ if and only if tworow $(S) \longrightarrow \operatorname{tworow}(T)$ in $L_{B}^{M o l}(b, 4)$. The Cartan matrix for $B_{2}$ used in [DLP1] is $\left(\begin{array}{cc}2 & -2 \\ -1 & 2\end{array}\right)$, so one must switch the colors on the edges of $L_{C_{2}}^{\beta \alpha}(\lambda)$ to get the same arrangement of edge colors in $L_{B}^{M o l}(b, 4)$. Theorem 2.1.Molev of [DLP1] shows that the edge-colored lattice $L_{B}^{M o l}(b, 4)$ is a positive rational supporting graph for a representation of $B_{2}$. Hence $L_{C_{2}}^{\beta \alpha}(\lambda)$ is a positive rational supporting graph for a representation of $C_{2}$ by Lemma 2.4.

Now let $\mathfrak{g}=G_{2}$. An argument similar to the previous paragraph using tableaux shows that when $\lambda=(a, 0)$, the lattice $L_{G_{2}}^{\beta \alpha}(\lambda)$ is the supporting graph $L_{G}^{L i t}\left(2, a \omega_{1}\right)$ from Corollary 3.3 of [DLP1]. For these $\lambda, L_{G_{2}}^{\beta \alpha}(\lambda)=L_{G_{2}}^{\alpha \beta}(\lambda)$. We note that the weight bases obtained in [DLP1] for these representations of $G_{2}$ were found by viewing $G_{2}$ as a Lie subalgebra of $B_{3}$ and considering the induced action of $G_{2}$ on an irreducible $B_{3}$-module of highest weight $a \omega_{1}$; it turns out that when this space is viewed as a $G_{2}$-module under the induced action, it remains irreducible. 
Figure 5.4: Coefficients for Proposition 5.6.

(In this figure, $A, B, C, D, E, p, q, r, s$ are nonnegative integers, and $p+q+r+s=1$.)
Edge $\mathbf{s} \stackrel{i}{\longrightarrow} \mathbf{t}$ in $L_{C_{2}}^{\beta \alpha}(1, b)$
Edge Coefficients

The next four edges have changes occurring in the two-row parts of the tableaux.

\begin{tabular}{|c|c|}
\hline 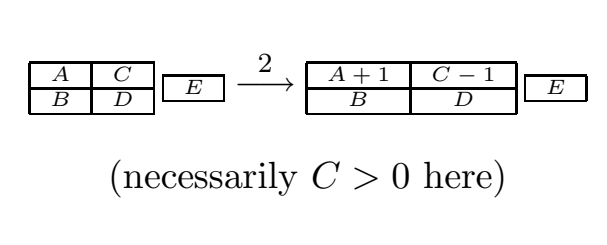 & $\begin{array}{c}c_{\mathbf{t}, \mathbf{s}}=\frac{C(2 B+2 C+2 D+2 E+1)}{2 C+2 D+2 E-1} \\
d_{\mathbf{s}, \mathbf{t}}= \\
\frac{(A+1)(2 C+2 E-1)(2 B+2 C+2 D+2 E+p+q+5 r+s-2)}{(2 C+2 D+2 E+1)(2 B+2 C+2 D+2 E+p+3 q+3 r+s-2)}\end{array}$ \\
\hline $\begin{array}{c}\begin{array}{|c|c|}A & C \\
B & D \\
\end{array} \stackrel{1}{\longrightarrow} \begin{array}{c|c|c|}A & C+1 \\
B & D \\
\end{array} \\
\text { (necessarily } E=1 \text { here) }\end{array}$ & $\begin{array}{c}c_{\mathbf{t}, \mathbf{s}}=(2 D+1)(2 B+2 D+2 q+2 r+4 s) \\
d_{\mathbf{s}, \mathbf{t}}=\frac{2(C+1)}{2 B+2 D+q+3 r+3 s}\end{array}$ \\
\hline 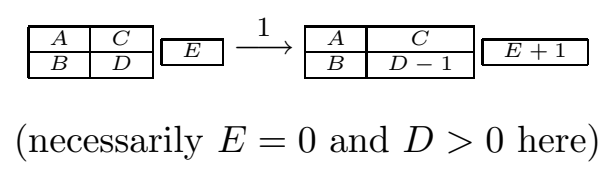 & $\begin{array}{c}c_{\mathbf{t}, \mathbf{s}}=2 D(2 C+1)(2 B+2 D+3 q+3 r+5 s-2) \\
d_{\mathbf{s}, \mathbf{t}}=\frac{1}{2 B+2 D+2 q+4 r+4 s-2}\end{array}$ \\
\hline 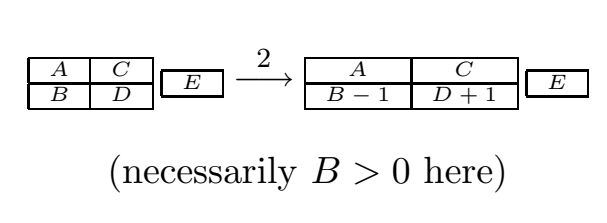 & $\begin{array}{c}c_{\mathbf{t}, \mathbf{s}}=\frac{B(B+1)(2 A+2 C+2 D+2 E+3)}{2 C+2 D+2 E+3} \\
d_{\mathbf{s}, \mathbf{t}}=\frac{(D+1)(2 D+2 E+1)}{(2 C+2 D+2 E+1)(B+s)}\end{array}$ \\
\hline
\end{tabular}

The next three edges have changes occurring in the one-row parts of the tableaux.

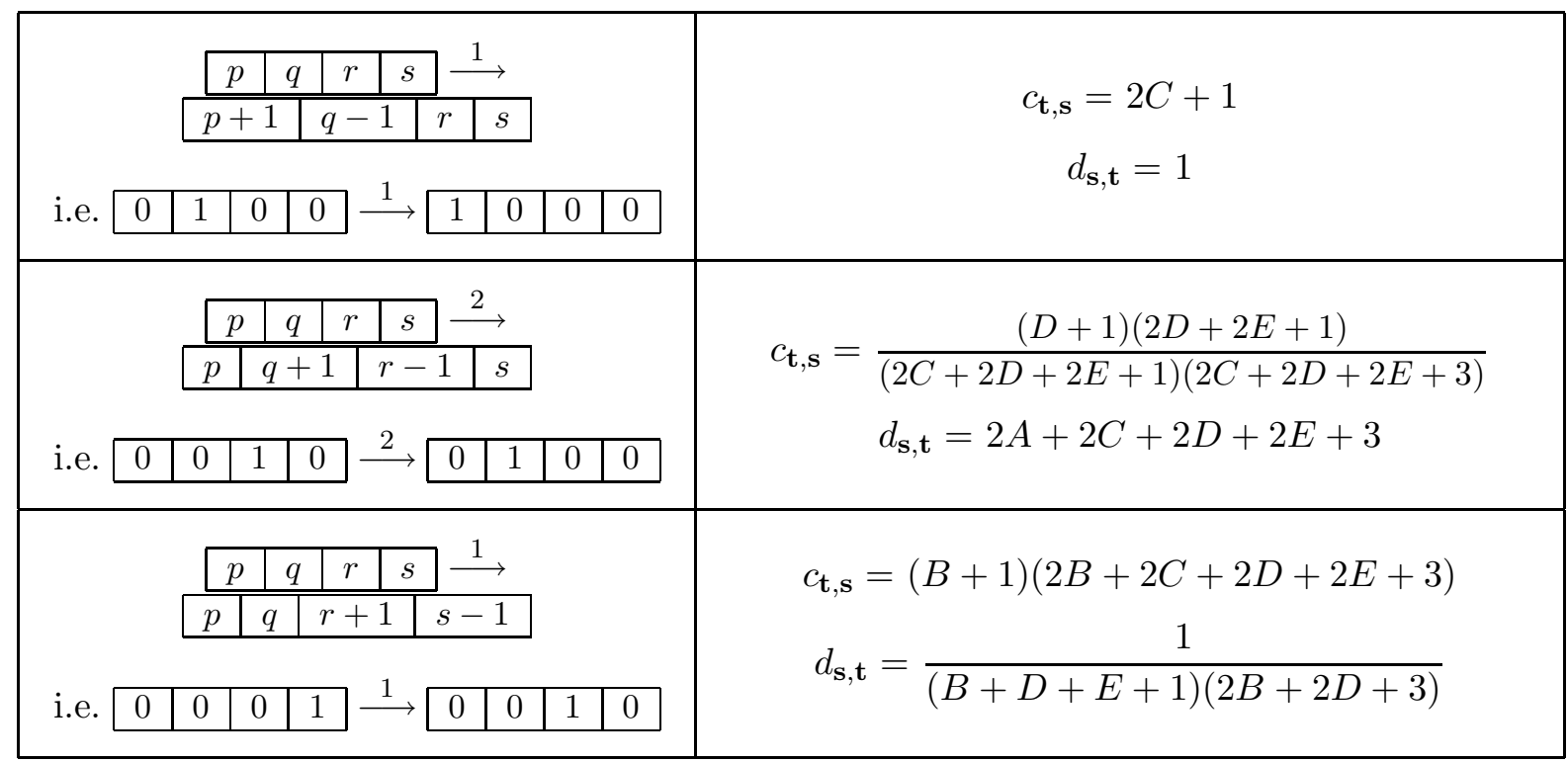


Figure 5.5: Possible ancestors and descendants of $S$ along edges of color 1. (This figure is referred to in the proof of Proposition 5.6; the tableau $S$ is from $L_{C_{2}}^{\beta \alpha}(1, b)$.)

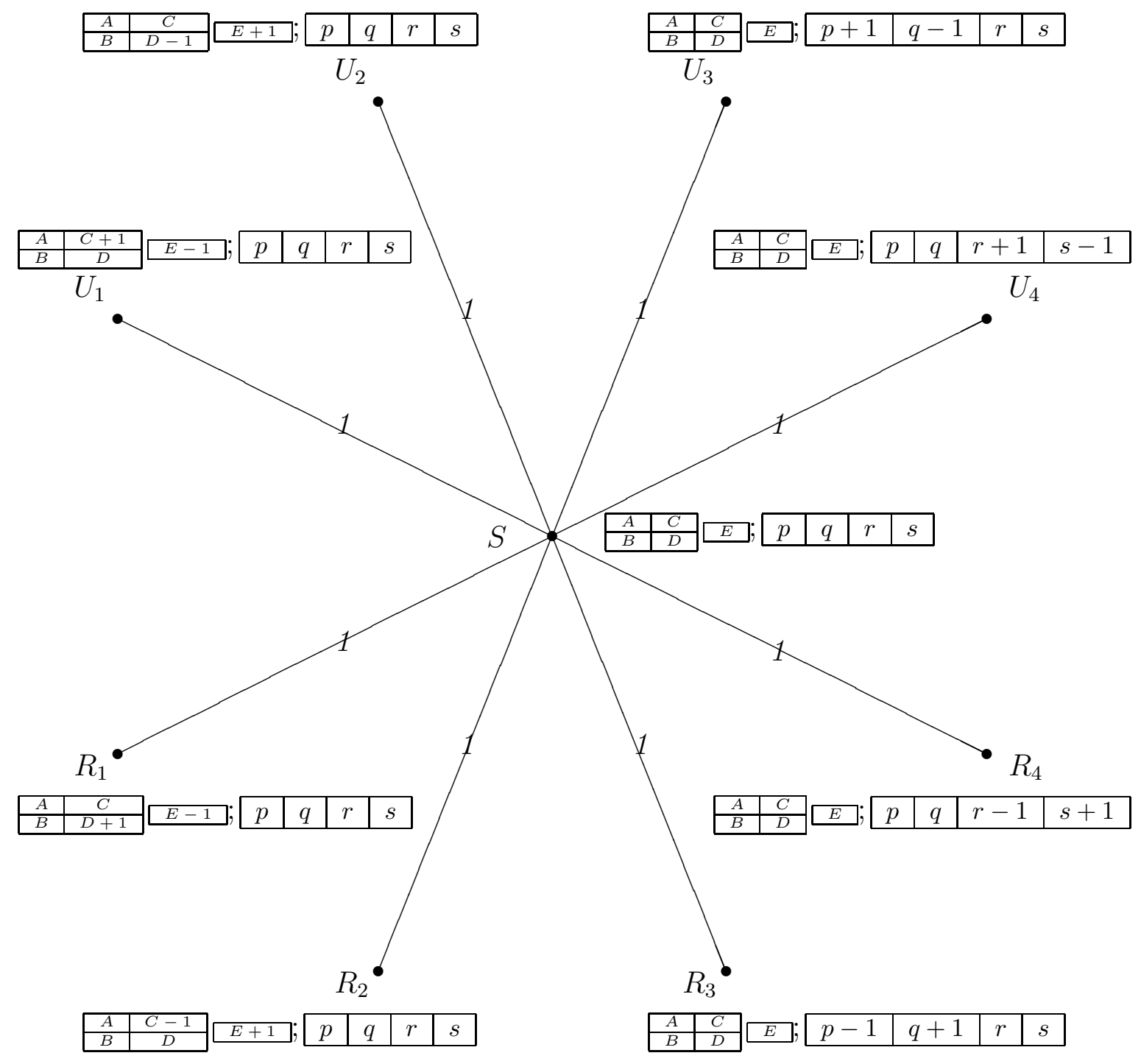

The edge $R_{1} \stackrel{1}{\rightarrow} S$ is present if and only if $E=1$ and $p=0$. The edge $R_{2} \stackrel{1}{\rightarrow} S$ is present if and only if $C>0, E=0$, and $p=0$. The edge $R_{3} \stackrel{1}{\rightarrow} S$ is present if and only if $B=0$, $D=0, E=0$, and $p=1$. The edge $R_{4} \stackrel{1}{\rightarrow} S$ is present if and only if $r=1$. The edge $S \stackrel{1}{\rightarrow} U_{1}$ is present if and only if $E=1$ and $p=0$. The edge $S \stackrel{1}{\rightarrow} U_{2}$ is present if and only if $D>0, E=0$ and $p=0$. The edge $S \stackrel{1}{\rightarrow} U_{3}$ is present if and only if $B=0, D=0$, $E=0$, and $q=1$. The edge $S \stackrel{1}{\rightarrow} U_{4}$ is present if and only if $s=1$. 
Figure 5.6: Possible ancestors and descendants of $S$ along edges of color 2 . (This figure is referred to in the proof of Proposition 5.6; the tableau $S$ is from $L_{C_{2}}^{\beta \alpha}(1, b)$.)

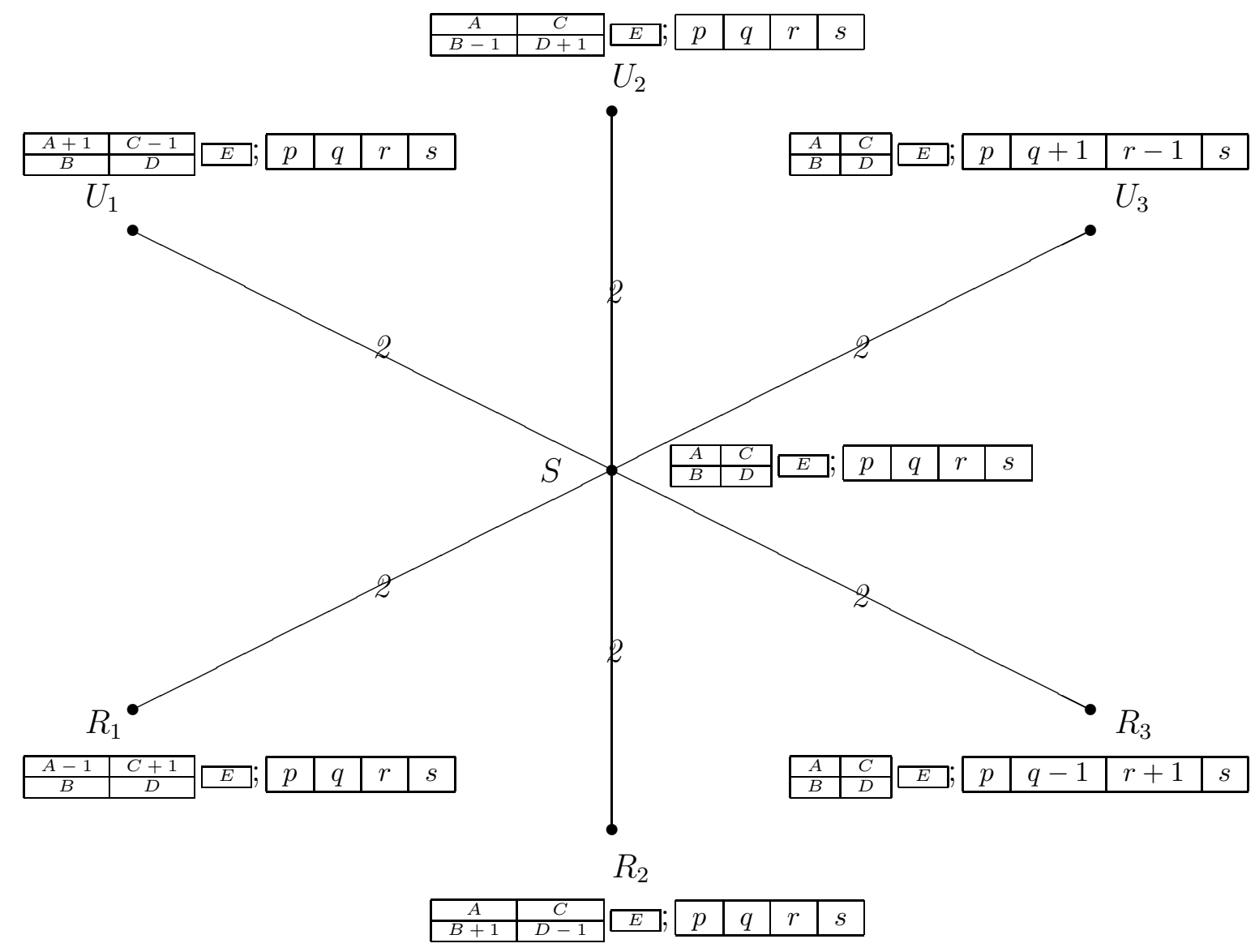

The edge $R_{1} \stackrel{2}{\rightarrow} S$ is present if and only if $A>0$. The edge $R_{2} \stackrel{2}{\rightarrow} S$ is present if and only if $D>0, p=0$, and $q=0$. The edge $R_{3} \stackrel{2}{\rightarrow} S$ is present if and only if $B=0$ and $q=1$. The edge $S \stackrel{2}{\rightarrow} U_{1}$ is present if and only if $C>0$. The edge $S \stackrel{2}{\rightarrow} U_{2}$ is present if and only if $B>0, p=0$, and $q=0$. The edge $S \stackrel{2}{\rightarrow} U_{3}$ is present if and only if $B=0$ and $r=1$. 
Figure 5.7: The top five ranks of $L_{C_{2}}^{\beta \alpha}(\lambda)$ for $\lambda=(a, b)$ with $a \geq 4, b \geq 4$.

(In the proof of Proposition 5.7, this subposet of $L_{C_{2}}^{\beta \alpha}(\lambda)$ is denoted $Q_{\lambda}$.)

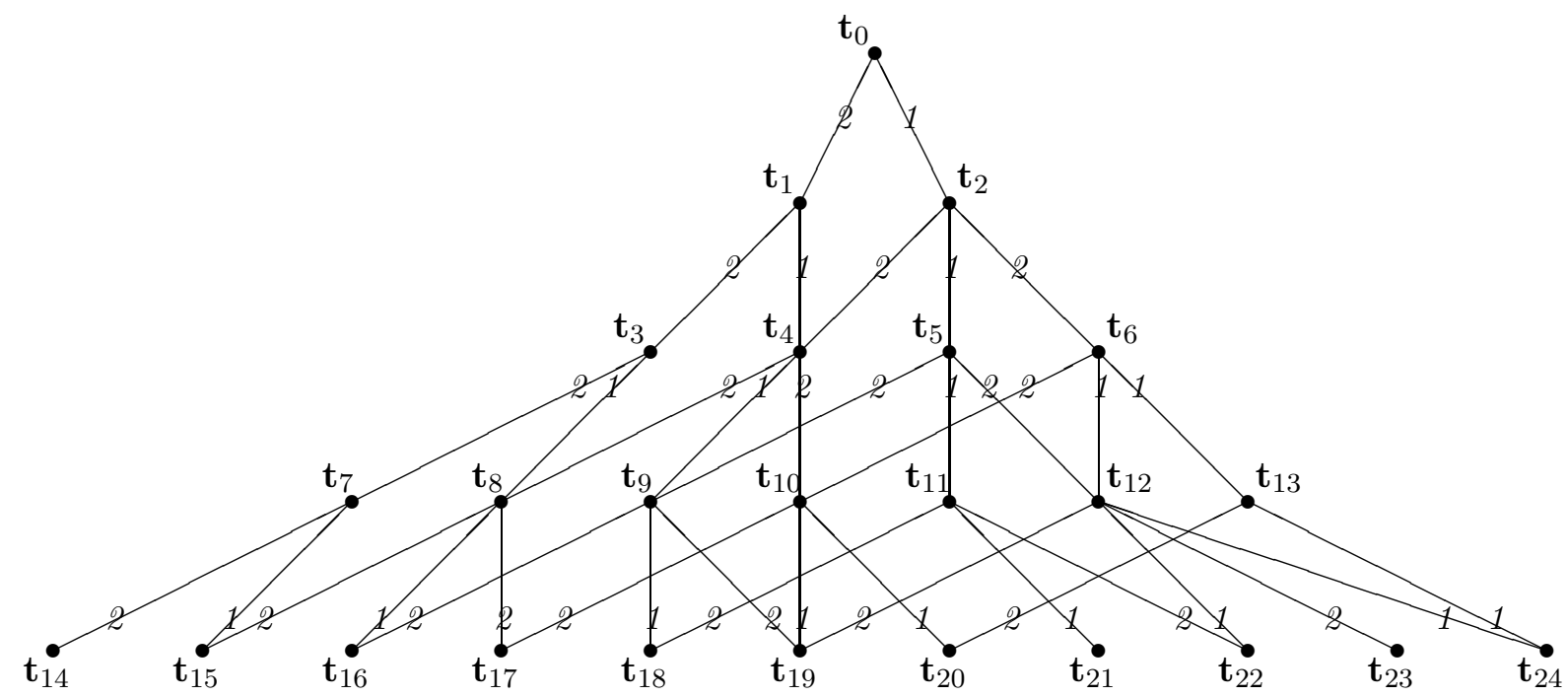

Figure 5.8: The top five ranks of $L_{G_{2}}^{\beta \alpha}(\lambda)$ for $\lambda=(a, b)$ with $a \geq 4, b \geq 4$.

(In the proof of Proposition 5.8, this subposet of $L_{G_{2}}^{\beta \alpha}(\lambda)$ is denoted $Q_{\lambda}$.)

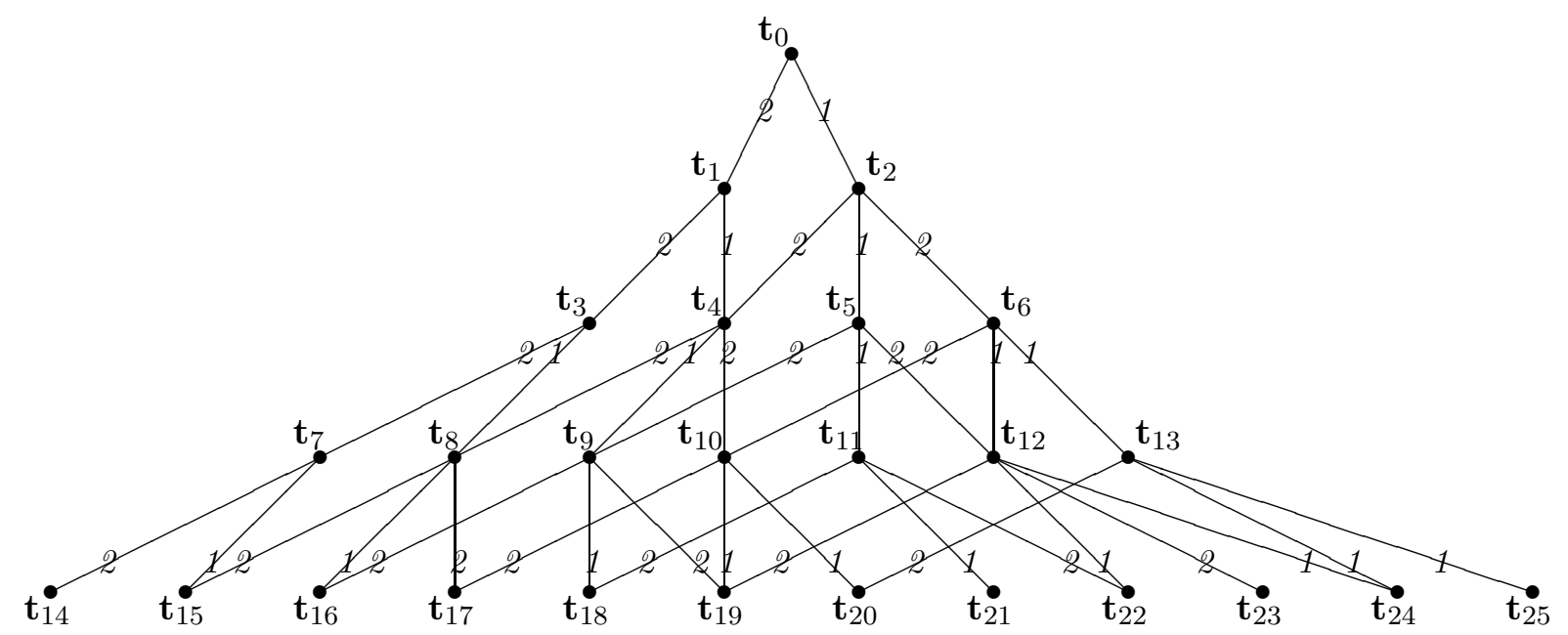


Figure 5.9: Vertices $\mathbf{t}_{i}$ in the top 5 ranks of a generic $a \geq 4, b \geq 4 C_{2^{-}}$or $G_{2^{-}}$-semistandard lattice.

\begin{tabular}{|c|c|c|c|c|c|c|}
\hline \multirow[t]{2}{*}{$l-\rho\left(\mathbf{t}_{i}\right)$} & \multirow[t]{2}{*}{$T_{i}=\operatorname{tableau}\left(\mathbf{t}_{i}\right)$} & \multirow[t]{2}{*}{$\begin{array}{l}\text { Dist. from } \\
\text { boundary }\end{array}$} & \multicolumn{2}{|c|}{$2 \rho_{1}\left(\mathbf{t}_{i}\right)-l_{1}\left(\mathbf{t}_{i}\right)$} & \multicolumn{2}{|c|}{$2 \rho_{2}\left(\mathbf{t}_{i}\right)-l_{2}\left(\mathbf{t}_{i}\right)$} \\
\hline & & & $C_{2}$ & $G_{2}$ & $C_{2}$ & $G_{2}$ \\
\hline 0 & $T_{0}={ }_{2}^{1} \ldots 1.11 \ldots 1$ & 0 & $a$ & $a$ & $b$ & $b$ \\
\hline \multirow[t]{2}{*}{1} & $T_{1}=\begin{array}{llllll}1 & \ldots & 1 & 1 & 1 & 1\end{array}$ & 0 & $a+2$ & $a+3$ & $b-2$ & $b-2$ \\
\hline & $T_{2}=\begin{array}{lllll}1 & \ldots 1 & 1 & \ldots & \ldots \\
2 & \ldots 2 & & & \\
\end{array}$ & 2 & $a-2$ & $a-2$ & $b+1$ & $b+1$ \\
\hline \multirow[t]{4}{*}{2} & $T_{3}=\begin{array}{llllllll}1 & \ldots & 1 & 1 & 1 & 1 & \ldots & \\
2 & \ldots & 2 & 3 & 3\end{array}$ & 0 & $a+4$ & $a+6$ & $b-4$ & $b-4$ \\
\hline & 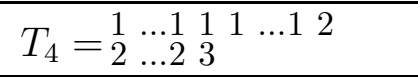 & 2 & $a$ & $a+1$ & $b-1$ & $b-1$ \\
\hline & 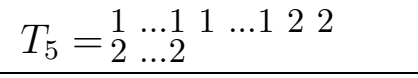 & 4 & $a-4$ & $a-4$ & $b+2$ & $b+2$ \\
\hline & 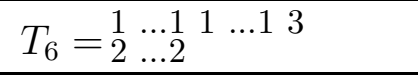 & 4 & $a$ & $a+1$ & $b-1$ & $b-1$ \\
\hline \multirow[t]{7}{*}{3} & 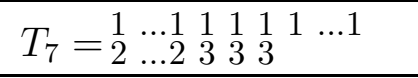 & 0 & $a+6$ & $a+9$ & $b-6$ & $b-6$ \\
\hline & 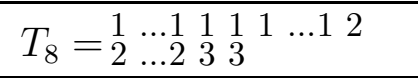 & 2 & $a+2$ & $a+4$ & $b-3$ & $b-3$ \\
\hline & 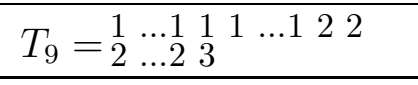 & 4 & $a-2$ & $a-1$ & $b$ & $b$ \\
\hline & 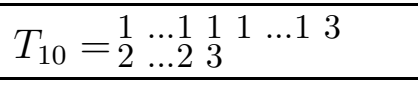 & 4 & $a+2$ & $a+4$ & $b-3$ & $b-3$ \\
\hline & 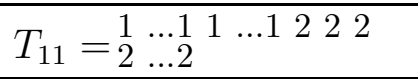 & 6 & $a-6$ & $a-6$ & $b+3$ & $b+3$ \\
\hline & 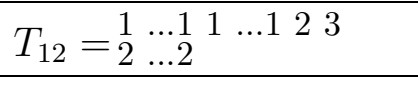 & 6 & $a-2$ & $a-1$ & $b$ & $b$ \\
\hline & 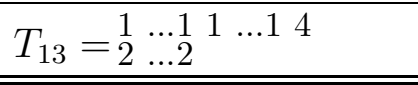 & 6 & $a-2$ & $a-1$ & $b$ & $b$ \\
\hline \multirow[t]{12}{*}{4} & $T_{14}=\begin{array}{lllllllll}1 & \ldots & 1 & 1 & 1 & 1 & 1 & 1 & \ldots \\
2 & \ldots & 2 & 3 & 3 & 3 & 3 & & \\
\end{array}$ & 0 & $a+8$ & $a+12$ & $b-8$ & $b-8$ \\
\hline & 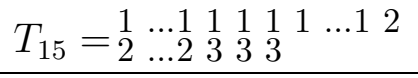 & 2 & $a+4$ & $a+7$ & $b-5$ & $b-5$ \\
\hline & $T_{16}=\begin{array}{llllllll}1 & \ldots & 1 & 1 & 1 & 1 & \ldots & 1 \\
2 & \ldots & 2 & 3 & 3\end{array}$ & 4 & $a$ & $a+2$ & $b-2$ & $b-2$ \\
\hline & $T_{17}=\begin{array}{lllllllll}1 & \ldots & 1 & 1 & 1 & 1 & \ldots & 1 & 3 \\
2 & \ldots & 2 & 3 & 3 & & & & \\
\end{array}$ & 4 & $a+4$ & $a+7$ & $b-5$ & $b-5$ \\
\hline & 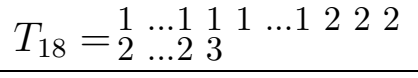 & 6 & $a-4$ & $a-3$ & $b+1$ & $b+1$ \\
\hline & 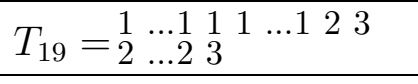 & 6 & $a$ & $a+2$ & $b-2$ & $b-2$ \\
\hline & $T_{20}=\begin{array}{lllllll}1 & \ldots & 1 & 1 & 1 & \ldots & 1 \\
2 & \ldots & 2 & 3 & & & \\
\end{array}$ & 6 & $a$ & $a+2$ & $b-2$ & $b-2$ \\
\hline & 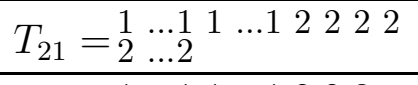 & 8 & $a-8$ & $a-8$ & $b+4$ & $b+4$ \\
\hline & 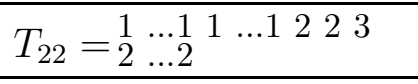 & 8 & $a-4$ & $a-3$ & $b+1$ & $b+1$ \\
\hline & 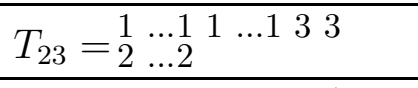 & 8 & $a$ & $a+2$ & $b-2$ & $b-2$ \\
\hline & 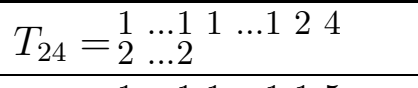 & 8 & $a-4$ & $a-3$ & $b+1$ & $b+1$ \\
\hline & 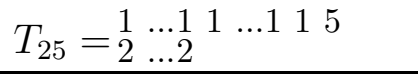 & 8 & NA & $a-2$ & NA & $b+1$ \\
\hline
\end{tabular}




\section{An additional example}

For $\lambda=(1,1)$, the $C_{2}$-semistandard poset $P_{C_{2}}^{\beta \alpha}(\lambda)$ is depicted in Figure 6.1 , and the 16-element $C_{2}$-semistandard lattice $L_{C_{2}}^{\beta \alpha}(\lambda)$ is also pictured. For $0 \leq i \leq 15$, set $T_{i}:=$ $\operatorname{tableau}\left(\mathbf{t}_{i}\right)$, where $\mathbf{t}_{0}<\mathbf{t}_{1}<\cdots<\mathbf{t}_{15}$ is the total ordering of elements of $L_{C_{2}}^{\beta \alpha}(\lambda)$ as defined in Section 3. These $C_{2}$-semistandard tableaux of shape $\lambda$ are: $T_{0}=\begin{array}{ll}1 & 1 \\ 2 & \text {, }\end{array}$

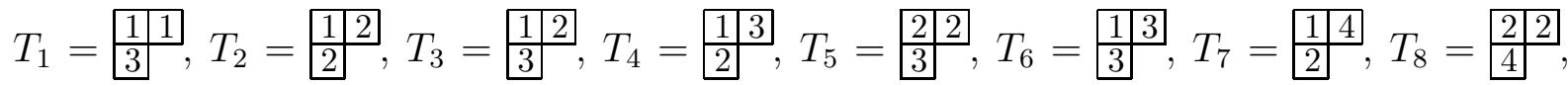

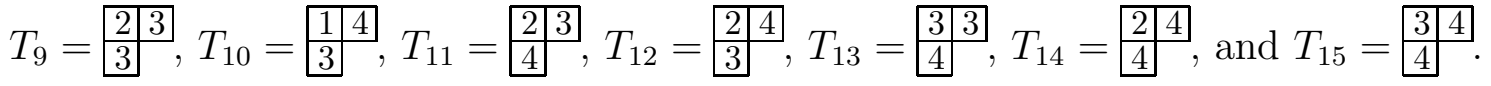

Figure 6.1: The $C_{2}$-semistandard poset $P_{C_{2}}^{\beta \alpha}(\lambda)$ and lattice $L_{C_{2}}^{\beta \alpha}(\lambda)$ for $\lambda=(1,1)$.

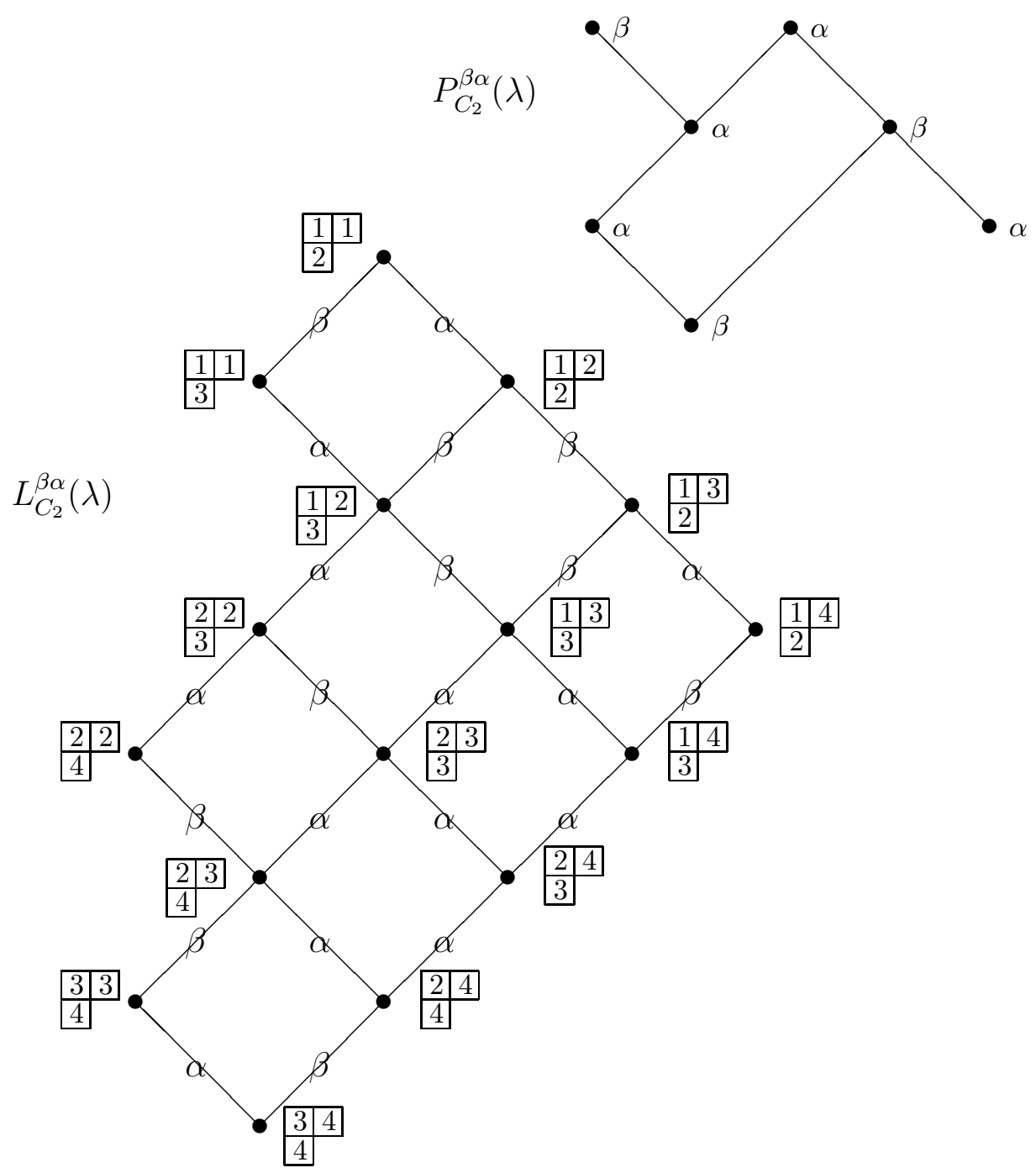




\section{References}

[Alv] L. W. Alverson II, "Distributive lattices and representations of the rank two simple Lie algebras," Master's thesis, Murray State University, 2003.

[ADLMPW] L. W. Alverson II, R. G. Donnelly, S. J. Lewis, M. McClard, R. Pervine, and N. J. Wildberger, "Distributive lattices defined for representations of rank two semisimple Lie algebras," preprint.

[Don1] R. G. Donnelly, "Extremal properties of bases for representations of semisimple Lie algebras," J. Algebraic Comb. 17 (2003), 255-282.

[Don2] R. G. Donnelly, "Extremal bases for the adjoint representations of the simple Lie algebras," Comm. Algebra, to appear.

[Don3] R. G. Donnelly, "A structure property for edge-colored ranked posets and some classification results," preprint.

[DLP1] R. G. Donnelly, S. J. Lewis, and R. Pervine, "Constructions of representations of $\mathfrak{o}(2 n+1, \mathbb{C})$ that imply Molev and Reiner-Stanton lattices are strongly Sperner," Discrete Math. 263 (2003), 61-79.

[DLP2] R. G. Donnelly, S. J. Lewis, and R. Pervine, "Solitary and edge-minimal bases for representations of the simple Lie algebra $G_{2}, "$ Discrete Math. , 306 (2006), $1285-1300$.

[GT] I. M. Gel'fand and M. L. Tsetlin, "Finite-dimensional representations of the group of unimodular matrices," Dokl. Akad. Nauk. USSR 71 (1950), 825-828. [Russian]

[Hum] J. E. Humphreys, Introduction to Lie Algebras and Representation Theory, Springer, New York, 1972.

[Mol1] A. Molev, "A basis for representations of symplectic Lie algebras," Comm. Math. Phys. 201 (1999), 591-618.

[Mol2] A. Molev, "Weight bases of Gel'fand-Tsetlin type for representations of classical Lie algebras," J. Phys. A: Math. Gen. 33 (2000), 4143-4158.

[Pr] R. A. Proctor, "Solution of a Sperner conjecture of Stanley with a construction of Gel'fand," J. Comb. Th. A 54 (1990), 225-234.

[Sta] R. P. Stanley, Enumerative Combinatorics, Vol. 1, Wadsworth and Brooks/Cole, Monterey, CA, 1986.

[Wil1] N. J. Wildberger, "A combinatorial construction for simply-laced Lie algebras," Adv. in Appl. Math. 30 (2003), 385-396.

[Wil2] N. J. Wildberger, "A combinatorial construction of $G_{2}$," J. Lie Theory $\mathbf{1 3}$ (2003), $155-165$. 